

\title{
Maximum likelihood and GMM estimation of dynamic panel data models with fixed effects
}

\author{
Hugo Kruiniger* \\ Queen Mary, University of London
}

October 1997, this version November 2000.

JEL classification: C11, C14, C23.

Keywords: dynamic panel data models, fixed effects, GMM, Conditional ML, Modified ML, Bayesian methods, (asymptotic) redundancy, Cramér-Rao and semiparametric efficiency bounds, unit root tests, parameter on boundary problem.

*Address: H.Kruiniger@QMW.AC.UK; Dept. of Economics, Mile End Road, London E1 4NS. This paper is a revised version of UCL WP 98-04 and other work. Versions of this paper have been presented at the Universities of Exeter and Cambridge, the econometrics seminar of LSE-STICERD, Queen Mary and Westfield College, the University of Southampton, ESEM99 in Santiago de Compostella, the 2000 Panel Data Conference in Geneva, and at the 2000 ESRC Econometrics Study Group Conference in Bristol. I should like to thank Richard Blundell, Grant Hillier, Jerzy Szroeter, and Elias Tzavalis for helpful comments and stimulating discussions. All errors are solely mine. This research was supported by the European Commission through Grant ERBFMBICT961594. 


\begin{abstract}
This paper considers inference procedures for two types of dynamic linear panel data models with fixed effects (FE). First, it shows that the closures of stationary ARMAFE models can be consistently estimated by Conditional Maximum Likelihood Estimators and it derives their asymptotic distributions. Then it presents an asymptotically equivalent Minimum Distance Estimator which permits an analytic comparison between the CMLE for the ARFE(1) model and the GMM estimators that have been considered in the literature. The CMLE is shown to be asymptotically less efficient than the most efficient GMM estimator when $N \rightarrow \infty$ but $T$ is fixed. Under normality some of the moment conditions become asymptotically redundant and the CMLE attains the Cramér-Rao lowerbound when $T \rightarrow \infty$ as well. The paper also presents likelihood based unit root tests. Finally, the properties of CML, GMM, and Modified ML estimators for dynamic panel data models that condition on the initial observations are studied and compared. It is shown that for finite $T$ the MMLE is less efficient than the most efficient GMM estimator.
\end{abstract}




\section{Introduction}

This paper studies estimation and testing procedures for two types of dynamic panel data models with fixed effects (FE): an ARFE(1) model that conditions on the initial observations irrespective of the value of the autoregressive parameter, $r$, and ARMAFE models that only condition on initial observations when the series contain a unit root but otherwise assume stationarity. As the models of the latter type are the closures of the stationary models, we will refer to them as inclusive ARMAFE models. The former model will be refered to as the conditional model. Throughout the paper we will assume cross-sectional independence. Furthermore we will assume that the errors are homoskedastic in the cross-sections. The second assumption is relaxed in Kruiniger (1998a, 1999).

In keeping with the traditional panel data literature, most of the asymptotic results in this paper are derived by letting the cross-sectional dimension, $N$, of the panel grow while the time dimension, $T$, is kept fixed. In a few cases we subsequently let the time dimension tend to infinity as well.

A considerable part of the paper is devoted to the study of the asymptotic properties of Conditional Maximum Likelihood (CML) Estimators (Rasch, 1961; Andersen, 1970, 1973). First the conditional likelihood functions corresponding to the inclusive ARMAFE models are shown to be differentiable at the unit root. Subsequently, the CML estimators for the inclusive ARMAFE models are shown to be consistent and their asymptotic distributions are derived. In contrast, the CMLE for the conditional ARFE(1) model that conditions on sufficient statistics for the individual mean parameters turns out to be inconsistent. This leads us to consider other likelihood based estimation methods for this model. Following a two-step Bayesian procedure, Lancaster (1997a,b) obtained a consistent estimator for this model. Lancaster observed that his estimator can also be viewed as a Modified Maximum Likelihood (MML) Estimator. We will provide an alternative consistency proof for this estimator ${ }^{1}$ and we will reinterpret Lancaster's estimator as a one-step Bayesian estimator. The

\footnotetext{
${ }^{1}$ Lancaster's argument for a unique maximum of the modified likelihood function is not adequate. In our proof we indicate how to select the maximum which is consistent when multiple maxima cannot be ruled out.
} 
asymptotic distribution of this estimator is also derived ${ }^{2}$. The asymptotic variances of both the CML estimators for the inclusive ARMAFE models and the MML estimator for the conditional ARFE(1) model are found to differ from the Cramér-Rao lower bounds when the time dimension of the panel is fixed.

The results obtained for the CML estimators of the inclusive ARMAFE models permit the formulation of unit root tests. These CMLE-based unit root tests will be compared with the unit root tests that have been proposed by Breitung and Meyer (1994) and Harris and Tzavalis (1999).

Then the paper presents Optimal Minimum Distance Estimators (OMDE's) (see also Chamberlain, 1982, 1984; Abowd and Card, 1989) for inclusive ARMAFE models which under normality are asymptotically equivalent to the CML estimators. The OMD estimator for the inclusive ARFE(1) model can more easily be compared with the (Optimal) GMM estimators for this model that have been proposed in the literature than the CMLE. We find that when $T$ is fixed, the Optimal Linear GMM estimator (Kruiniger, 2000) which exploits all the second moment conditions is (generally) asymptotically more efficient than the CMLE. This is due to the fact that the former estimator also exploits information in the levels of the data.

Along the lines of Ahn and Schmidt (1995) the Optimal Linear GMM estimator can be shown to attain the semiparametric efficiency bound corresponding to the ARMAFE model that is characterized by all the restrictions on the second moments that are implied by stationarity. Next, we show that under the assumption of normality both the CMLE and the Optimal Linear GMM estimator for the stationary ARMAFE model attain the Cramér-Rao lower bound when $T$ tends to infinity. It follows that under normality some of the moment conditions that are exploited by the Optimal Linear GMM estimator are asymptotically redundant (cf the notion of redundancy as introduced by Breusch et al. 1999). We give a formal definition of asymptotic redundancy and formulate a necessary and sufficient condition for asymptotic redundancy.

We also consider GMM estimation of the conditional model. A subset of the moment conditions for the inclusive model remains valid for this model. As in the stationary case,

\footnotetext{
${ }^{2}$ Lancaster (1997b) has independently derived the asymptotic distribution of the MMLE.
} 
the Optimal GMM Estimator that exploits all the second moment conditions is found to be asymptotically at least as efficient and in some cases, e.g. under normality, more efficient than the likelihood-based alternative, i.e. the Modified ML Estimator, when $T$ is fixed.

It is well known that unconditional maximum likelihood estimators of dynamic panel data models with fixed individual effects are inconsistent both when the initial observations are taken as given and in the stationary model. The breakdown of the unconditional MLE is due to the presence of incidental parameters (see Neyman and Scott (1948) and Nickell (1981)). MaCurdy (1981a, 1982) suggested that the ML method does yield consistent estimates for stationary ARMAFE models when the data are first differenced but his works lack a rigorous proof $^{3}$. Moreover, MaCurdy did not consider the unit root case. We will see that the CMLE for the inclusive ARMAFE model is equal to the First Differences ML estimator (FDMLE).

So far application of CML methods to these models has not been considered in the literature. Kiefer (1980) discusses GLS estimation of panel data models with fixed effects and arbitrary, unknown intertemporal covariance matrix, say $V$, and gives a CML 'interpretation' to the fully iterated version of his GLS estimator ${ }^{4}$. He estimates the covariance matrix for transformed data, i.e. $Q V Q$, where $Q$ is the within matrix $\left(I_{T-\frac{1}{T}} \iota \iota^{\prime}\right)$. As he does not impose any structure on the covariance matrix, e.g. the structure corresponding to an autoregressive process, he cannot consistently estimate the covariance matrix itself. However, he only aims for asymptotically efficient estimators for the slope parameters and is not interested in estimates of $V$ itself. In contrast, in the case of the stationary ARMAFE model we try to estimate the parameters of a restricted version of $V$. Nonetheless, our CMLE for the inclusive model and Kiefer's CMLE are related as they condition on the same sufficient statistics for the individual means.

The generalisation of the results on the asymptotic properties of the CMLE for the inclusive ARFE(1) model to CML estimators for inclusive ARMAFE models of arbitrary lag orders relies heavily on important results that have been derived by Van der Leeuw (1994).

\footnotetext{
${ }^{3}$ Our proofs of consistency and asymptotic normality of the CMLE's rely heavily on results obtained by Van der Leeuw (1994). These results allow us to prove continuity and differentiability of the likelihood functions.

${ }^{4}$ Kiefer states without proof that the iterated GLS estimator converges to the CML estimator. Actually, this follows from a result in Oberhofer and Kmenta (1974).
} 
In particular, he obtained a closed form representation of the covariance matrix of stationary ARMA errors.

In the study of the CML estimators for the inclusive ARMAFE models, the unit root case poses several technical problems. First the covariance matrix of the ARMA errors, $V$, no longer exists. Secondly, since the unit root is on the boundary of the parameter space, deriving the asymptotic distributions of the CML estimators is not straightforward (see e.g. Andrews, 1999). Making use of the results of Van der Leeuw we first show that the covariance matrix of the first-differenced errors is left-continuous at the unit root. Therefore at the unit root $D V D^{\prime}$ still exists and is a covariance matrix of stationary errors (the first-difference matrix $D$ is defined in section 2). Subsequently, we are able to show that the conditional likelihood function is differentiable at the unit root.

We find that our CMLE for the conditional model is different from the 'within' ML estimator for this model. However, neither the CMLE nor the Within MLE for this model are consistent when $T$ is kept fixed although the incidental mean parameters are no longer present in the conditional and within likelihood functions. In this connection we note that the number of initial conditions also grows with $N$ and that the initial conditions remain important when $T$ is kept fixed.

The paper is organised as follows. Section 2 discusses CML estimation of the inclusive ARFE(1) model. Section 3 discusses estimation of the conditional ARFE(1) model. Section 4 generalizes the results in section 2 to inclusive ARMAFE models. Sections 3 and 4 also compare ML-type and GMM estimators in terms of efficiency. Both section 2 and section 4 propose tests of the unit root hypothesis versus stationarity. Section 5 concludes. 


\section{CML estimation of dynamic panel data models: the stationary case and the unit root case}

In this section and section 3 we consider estimation of parameter $r$ in two versions of the AR(1) model with Fixed Effects, henceforth abbreviated as ARFE(1)

$$
\begin{aligned}
y_{i, t} & =r y_{i, t-1}+(1-r) \mu_{i}+\varepsilon_{i, t} \\
\check{\mu}_{i} & =(1-r) \mu_{i} \\
\varepsilon_{i, t} \mid \check{\mu}_{i}, y_{i, t_{0}} & \sim N\left(0, \sigma^{2}\right) \quad(i . i . d .), \quad t=t_{0}+1, \ldots, T \\
t_{0} & \leq 1
\end{aligned}
$$

where $i \in\{1,2, \ldots, N\}$ indicates the individual unit and $t$ indicates the time period. For each individual unit we have $T$ observations on $y: y_{i, 1}, \ldots, y_{i, T}$. The designation "fixed effects" should be taken as to reflect the absence of any distributional assumptions about the individual effects $\mu_{i}$, except that they are independent of the disturbances $\varepsilon_{i, t}$ given the initial (possibly unobserved) realization of the process $y_{i, t_{0}}{ }^{5}$. The i.i.d. assumption applies to both the time-series dimension and the cross-section dimension of the panel.

We focus first on the ARFE(1) model as our results can most easily be shown for this model but as we will see in section 4 generalizations of the results to more complicated models such as ARMAFE(p,q) models are possible although more involving ${ }^{6}$.

We will consider estimation of two versions of the $\operatorname{ARFE}(1)$ model. The versions differ with regard to the assumptions that are made about the initial conditions and the set of admissible values for $r$. In section 3 we will discuss estimation of the ARFE(1) model conditional on the initial observations. However, if the distribution of the initial observations is known, conditioning on these observations when estimating the parameters would entail

\footnotetext{
${ }^{5}$ Balestra and Nerlove (1966) consider ML estimation of ARXEC models, where the individual effects are assumed to be normally distributed as well. Anderson and Hsiao $(1981,1982)$ also discuss ML estimation of various ARXEC models. Blundell and Smith (1991) considered a range of CML estimators for ARXEC models.

${ }^{6}$ Kruiniger (1998a, 1999) consider (C)ML and GMM estimation of extensions of these models that allow for fixed individual effects in the variance as well.
} 
a loss of information and as a consequence the estimator obtained would (in general) be less efficient. An important case where the distribution of the initial observations is known is the situation where the data follow a stationary process, i.e. $|r|<1$. This assumption about the data is often satisfied. We will discuss ML type estimation methods for the stationary ARFE(1) model in this section. We will also study the unit root case even though that model belongs to the class of conditional models, and we will discuss the possibility of testing the unit root hypothesis against stationary alternatives.

We will now state the stationarity assumptions

$$
\begin{array}{ll}
\text { STAT1: } & |r|<1 \\
\text { STAT2: } & y_{i, t_{0}} \sim N\left(0, \frac{\sigma^{2}}{1-r^{2}}\right)
\end{array}
$$

Assumptions STAT1-STAT2 imply that $\left\{y_{i, t}\right\}$ is stationary.

We will first consider unconditional ML estimation of the stationary model. Before we can define the unconditional MLE's, we have to introduce more notation. Let $y_{i}$ denote the $T$-vector of all the observations including the first observation for individual $i$. If we denote the covariance matrix of the $y_{i}^{\prime} s$ by $\sigma^{2} V_{T}(r)$, we can restate the stationary version of model (1) as

$$
\left(y_{i}-\mu_{i} \iota\right) \equiv u_{i} \sim N\left(0, \sigma^{2} V_{T}(r)\right), i=1, \ldots, N,(i . i . d .)
$$

where $\iota$ is a $T$-vector of ones and $V_{T}$ has a specific $\operatorname{AR}(1)$ structure 78

$$
V_{T}(r)=\frac{1}{1-r^{2}}\left(\begin{array}{ccccccc}
1 & r & r^{2} & . & . & . & r^{T-1} \\
r & 1 & r & . & . & . & r^{T-2} \\
r^{2} & r & 1 & . & & & . \\
. & . & . & . & . & & . \\
. & . & & . & . & . & . \\
\cdot & . & & & . & . & r \\
r^{T-1} & r^{T-2} & . & . & . & r & 1
\end{array}\right) \text { with }|r|<1
$$

\footnotetext{
${ }^{7}$ We can rewrite an ARXFE(1) model in this form, which does not involve lagged observations, only if the parameters of the exogenous variables in the dynamic form (cf. (1)) satisfy COMFAC restrictions.

${ }^{8}$ We will drop the subscript $T$ when knowledge of the time-dimension of the panel is unimportant. We will also omit the argument $r$ of $V$.
} 
The unconditional MLE's for the triple $\left\{r, \mu, \sigma^{2}\right\}$ in the stationary model are the statistics that maximize the log-likelihood function

$$
\begin{aligned}
& l_{U}\left(y_{i} ; r, \mu, \sigma^{2}\right)=-\frac{N T}{2} \log (2 \pi)-\frac{N T}{2} \log \sigma^{2} \\
& -\frac{N}{2} \log \left|V_{T}(r)\right|-\frac{1}{2 \sigma^{2}} \sum_{i=1}^{N}\left(\left(y_{i}-\mu_{i} \iota\right)^{\prime} V_{T}^{-1}(r)\left(y_{i}-\mu_{i} \iota\right)\right)
\end{aligned}
$$

For finite $T$ these estimators for $\left\{r, \sigma^{2}, \mu\right\}$ are inconsistent due to the incidental parameters problem (see Neyman and Scott (1948)).

We will now explore whether the parameters of the stationary ARFE(1) model can be estimated consistently by employing the Conditional Maximum Likelihood (CML) method.

Kiefer (1980) has shown that

$$
\widetilde{\mu}_{i}=\frac{\iota^{\prime} V^{-1} y_{i}}{\iota^{\prime} V^{-1} \iota}
$$

is a minimal sufficient 'statistic' for $\mu_{i}$, where in fact $V$ could be any intertemporal covariance matrix.

The conditional log-likelihood function, i.e. the logarithm of the conditional density of the $y_{i}^{\prime} s$ given the $\widetilde{\mu}_{i}^{\prime} s$, is given by

$$
\begin{aligned}
& l_{C}\left(y_{i} ; r, \sigma^{2} \mid \widetilde{\mu}_{i}\right)=-\frac{N(T-1)}{2} \log (2 \pi)-\frac{N(T-1)}{2} \log \sigma^{2} \\
& -\frac{N}{2} \log \left|V-\frac{\iota \iota^{\prime}}{\iota^{\prime} V^{-1} \iota}\right|^{+}-\frac{1}{2 \sigma^{2}} \sum_{i=1}^{N}\left(\left(y_{i}-\mu_{i}\right)^{\prime}\left(V^{-1}-\frac{V^{-1} \iota^{\prime} V^{-1}}{\iota^{\prime} V^{-1} \iota}\right)\left(y_{i}-\mu_{i}\right)\right)
\end{aligned}
$$

where $|M|^{+}$denotes the product of the nonzero eigenvalues of $M$ (see Rao (1973) pp. 527$528)$.

The CML estimators for the pair $\left\{r, \sigma^{2}\right\}$ are equal to the statistics that maximize (6).

MaCurdy (1981a, 1982) has suggested to take first differences of the data, such that the individual effects drop out from the model, before applying the the ML method.

Let $D_{T}$ denote the following $(T-1 \times T)$ matrix

$$
D_{T}=\left(\begin{array}{ccccccc}
-1 & 1 & 0 & . & . & . & 0 \\
0 & -1 & 1 & 0 & & & . \\
. & 0 & -1 & 1 & 0 & & . \\
. & & . & . & . & . & . \\
. & & & . & . & . & 0 \\
0 & . & . & . & 0 & -1 & 1
\end{array}\right)
$$


Then we obtain

$$
D\left(y_{i}-\mu_{i} \iota\right)=D y_{i}=D u_{i} \sim N\left(0, \sigma^{2} D V D^{\prime}\right), i=1, \ldots, N,(\text { i.i.d. })
$$

The covariance matrix of $D y_{i}, \sigma^{2} D V D^{\prime}$, has an $\operatorname{ARMA}(1,1)$ structure with known MA parameter $(-1)$.

The First Difference MLE for the triple $\left\{r, \mu, \sigma^{2}\right\}$ globally maximizes ${ }^{9} 10$

$$
\begin{aligned}
& l\left(D y_{i} ; r, \mu, \sigma^{2}\right)=-\frac{N(T-1)}{2} \log (2 \pi)-\frac{N(T-1)}{2} \log \sigma^{2} \\
& -\frac{N}{2} \log \left|D V D^{\prime}\right|-\frac{1}{2 \sigma^{2}} \sum_{i=1}^{N}\left(\left(y_{i}-\mu_{i}\right)^{\prime} D^{\prime}\left(D V D^{\prime}\right)^{-1} D\left(y_{i}-\mu_{i}\right)\right)
\end{aligned}
$$

Kiefer has argued that $L_{C}\left(Q y_{i} \mid \widetilde{\mu}_{i}\right) \propto L_{C}\left(y_{i} \mid \widetilde{\mu}_{i}\right)$, where $Q$ is the Within matrix and $L=$ $\exp (l)$, and it can likewise be shown that $L_{C}\left(D y_{i} \mid \widetilde{\mu}_{i}\right) \propto L_{C}\left(y_{i} \mid \widetilde{\mu}_{i}\right)$. However, $L_{C}\left(D y_{i} \mid \widetilde{\mu}_{i}\right)=$ $L\left(D y_{i}\right)^{11}$. So we find that the CMLE is equal to the FDMLE ${ }^{12}$.

Sofar we have maintained that $|r|<1$. We will now consider the case where $r=1$. The unit root case is special because no matter how long ago the process $\left\{y_{i, t}\right\}$ has started, the influence of early realisations of $y$ and $\varepsilon$ on the current observation $y_{i, t}$ does not vanish ${ }^{13}$. As a consequence, when $r=1$, we cannot avoid conditioning on the initial observations. Moreover, we cannot rewrite model (1) as (2). However, the conditional log-likelihood function (6) is still well-defined for $r=1$. In particular, in appendix A it will be shown that $E\left(D y_{i} y_{i}^{\prime} D^{\prime}\right)$ is

${ }^{9}$ It is easy to show that when $\sigma^{2}$ is unknown the FDMLE for $r$ in the $\operatorname{AR}(1)$ model only exists for $T \geq 3$ (see also Lancaster and Lindenhovius (1996)).

${ }^{10} \mathrm{MaCurdy}(1981,1982)$ does not rigorously prove that the FDMLE is consistent.

${ }^{11}$ There is another way of showing that $L_{C}\left(y_{i} \mid \widetilde{\mu}_{i}\right) \propto L\left(D y_{i}\right)$. Lancaster and Lindenhovius have shown that $V^{-1}-\frac{V^{-1} \iota^{\prime} V^{-1}}{\iota^{\prime} V^{-1} \iota}=D^{\prime}\left(D V D^{\prime}\right)^{-1} D$. Furthermore, we can find a constant $T \times(T-1)$ orthonormal matrix $\check{D}^{\prime}$ that satisfies $\check{D} \iota_{T}=0$ by performing Gram-Schmidt orthogonalization on $D^{\prime}$. Clearly, there exists a constant nonsingular matrix $\check{S}$, such that $\check{D}=\check{S} D$. Then following Rao $\left(1973\right.$, p. 528) $\left|V-\frac{\iota \iota^{\prime}}{\iota^{\prime} V^{-1} \iota}\right|^{+}=\mid \check{D}\left(V-\frac{\iota \iota^{\prime}}{\iota^{\prime} V^{-1} \iota} \check{D}^{\prime}\left|=\check{c}^{2}\right| D V D^{\prime} \mid\right.$ where $\check{c}=|\check{S}| \neq 0$.

${ }^{12}$ See Kruiniger (1998a) for the relationship between CML and Transformed ML estimators.

${ }^{13}$ Another consequence of $r=1$ is of course that the incidental parameters $\mu_{i}$ drop out from the model, so in this case there is no need for removing them. 
(left-)continuous in $r=1$ and that the conditional likelihood can be differentiated as many times as is needed in (the neighbourhood of) $r=1$.

Therefore we can consider estimation of a model that includes both the stationary model and the unit root case. We first state the following

Definition 1 The inclusive ARFE(1) model is characterized by (1) and the following assumptions:

INCL1: $\quad-1<r \leq 1$

INCL2: $\quad y_{i, t_{0}} \sim N\left(0, \frac{\sigma^{2}}{1-r^{2}}\right)$ if $-1<r<1 ;\left|y_{i, t_{0}}\right|<\infty$ if $r=1$

We can now propose the following

Theorem 1 The CML estimator for $r$ and $\sigma^{2}$ in the inclusive ARFE(1) model, which maximizes (6), is consistent.

\section{PROOF}

See appendix A. In the proof we pay special attention to the unit root case. Andersen (1970) has only proven consistency of CMLE's that make use of true sufficient statistics which do not depend on parameters.

The result in theorem 1 suggests a new unit root test for panel data models with fixed $T$ which is simply based on the CMLE for $r$ and knowledge of its large- $N$ asymptotic distribution.

Since $\widehat{r}_{C M L}$ is a function of cross-sectional averages of the data, the finite sample properties of this estimator are expected to be quite different from those of an ML/LS estimator of $r$, which is based on a single time-series; in particular, averaging across the cross-sectional units mitigates the bias which in the case of an estimator based on a single time-series would exist because of the correlation between the numerator and the denominator.

We will now derive the asymptotic distribution of the CMLE (FDMLE). We will make use of the second Bartlett identity and the fact that the Expected Hessian (EH) of the 
conditional log-likelihood is equal to the difference between the EH of the unconditional loglikelihood (EHU) and the $\mathrm{EH}$ of the log-likelihood of the conditioning statistic, $\widetilde{\mu}_{i},(\mathrm{EHM})$ 14. We now state

Theorem 2 The large $N$ (fixed T) asymptotic distribution of $\left(\widehat{r}_{C M L}, \widehat{\sigma}_{C M L}^{2}\right)$ is given by

$$
\sqrt{N}\left(\begin{array}{c}
\widehat{r}_{C M L}-r \\
\widehat{\sigma}_{C M L}^{2}-\sigma^{2}
\end{array}\right) \rightarrow N\left(0,\left(\lim _{\breve{r} \rightarrow r}\left(E H M_{\breve{r}, \sigma^{2}}-E H U_{\breve{r}, \sigma^{2}}\right)\right)^{-1}\right)
$$

where

$$
\begin{gathered}
E H M_{r, \sigma^{2}}-E H U_{r, \sigma^{2}}=\left(\begin{array}{cc}
I_{r r} & -\frac{(T-1)}{(1+r)(T(1-r)+2 r) \sigma^{2}} \\
-\frac{(T-1)}{(1+r)(T(1-r)+2 r) \sigma^{2}} & \frac{T-1}{2 \sigma^{4}}
\end{array}\right) \\
I_{r r}=\frac{-2(1+r)\left(r^{T-1}-2(T-2) r^{2}+2 T-3\right)(T(1-r)+2 r)}{\left(1-r^{2}\right)^{2}(T(1-r)+2 r)^{2}} \\
+\frac{2(1+r)^{2}((T-2)(r-1)-1)^{2}+\left((T-2)\left(1-r^{2}\right)+1+r^{2}\right)(T(1-r)+2 r)^{2}}{\left(1-r^{2}\right)^{2}(T(1-r)+2 r)^{2}}
\end{gathered}
$$

\section{PrOOF}

See appendix A. We note that both EHU and EHM are block diagonal.

It can easily be verified that the asymptotic covariance matrix is positive definite symmetric for $-1<r<1$ (see also section 4 and appendix A.4).

In what follows we will initially assume that $r<1$ but we will discuss the case $r=1$ later on. It is easy to verify that for finite $T$ the CMLE for $\left(r, \sigma^{2}\right)$ in the ARFE(1) model does not attain the Cramér-Rao lower bound (hereafter CR bound, $C R(r, T))-\left(E H U_{r, \sigma^{2}}\right)^{-1} 15$ 16. The reason is that the conditioning statistic $\widetilde{\mu}_{i}$ contains information about the common parameters $r$ and $\sigma^{2}$.

\footnotetext{
${ }^{14}$ We have by definition $L\left(\bar{y}_{i} \mid D y_{i}\right) L\left(D y_{i}\right)=L\left(y_{i}\right)$. Because we can easily show that $L\left(\widetilde{\mu}_{i}\right)=$ $L\left(\bar{y}_{i} \mid D y_{i}\right)$, it follows that $\left(L_{C}\left(y_{i} \mid \widetilde{\mu}_{i}\right) \propto\right) L\left(D y_{i}\right)=L\left(y_{i}\right) / L\left(\widetilde{\mu}_{i}\right)$. Alternatively, it follows from the proof of the second theorem in Lancaster and Lindenhovius that $\left|D V D^{\prime}\right|=\frac{\iota^{\prime} V^{-1} \iota}{\left|V^{-1}\right|}$ (in fact for arbitrary $V)$. Therefore, we have again that $L\left(D y_{i}\right)=L\left(y_{i}\right) / L\left(\widetilde{\mu}_{i}\right)$.

${ }^{15}$ The Information Matrix equals minus EHU.

${ }^{16}$ For instance, the term $\frac{2}{1-r^{2}}\left(r^{T-1}\right)$ from $\iota^{\prime} \frac{d V^{-1}}{d r} V \frac{d V^{-1}}{d r} \iota$ in EHM does not cancel out. Since the $\operatorname{ARFE}(1)$ model is just a special case of the $\operatorname{ARMAFE}(\mathrm{p}, \mathrm{q})$ model, the CMLE's for more general models are not asymptotically efficient either.
} 
The fact that the CMLE for common parameters in a model with incidental parameters does not attain the CR bound for large $N$ but finite $T$ is not an exception ${ }^{17}$. We will see in section 4 that a more efficient root- $N$ consistent estimator exists for the inclusive model.

When $T \rightarrow \infty$, the conditioning statistic becomes unimportant and the CMLE converges to the unconditional MLE, which maximizes (4). Although the unconditional MLE is inconsistent for large $N$ but finite $T$, it is consistent for large $T$. For large $T$ the asymptotic distribution of both estimators for the stationary ARFE(1) model is given by

$$
\sqrt{N T}\left(\begin{array}{c}
\widehat{r}_{C M L}-r \\
\widehat{\sigma}_{C M L}^{2}-\sigma^{2}
\end{array}\right) \rightarrow N\left(\left(\begin{array}{l}
0 \\
0
\end{array}\right),\left(\begin{array}{cc}
1-r^{2} & 0 \\
0 & 2 \sigma^{4}
\end{array}\right)\right)
$$

The asymptotic covariance matrix equals $\lim _{T \rightarrow \infty}-T\left(E H U_{r, \sigma^{2}}\right)^{-1}$ which is the CR bound.

When $r=1$ we have the following

Theorem 3 When $r=1$ and $T$ is fixed, the (large $N$ ) asymptotic distribution of the CMLE is given by

$$
\sqrt{N}\left(\begin{array}{c}
\widehat{r}_{C M L}-1 \\
\widehat{\sigma}_{C M L}^{2}-\sigma^{2}
\end{array}\right) \rightarrow N\left(0, \frac{1}{T-2}\left[\begin{array}{cc}
\frac{8}{(T-1)} & 4 \frac{\sigma^{2}}{(T-1)} \\
4 \frac{\sigma^{2}}{(T-1)} & 2 \sigma^{4}
\end{array}\right]\right)
$$

\section{PROOF}

See appendix A. We obtain $\lim _{r \uparrow 1}\left(E H M_{r, \sigma^{2}}-E H U_{r, \sigma^{2}}\right)$ by applying de l'Hôpital's first rule twice.

We stress that this result depends on the assumption that $T$ is kept finite. If both $T$ and $N \rightarrow \infty$, the asymptotic distribution of the CMLE depends on which dimension increases faster or first (see also Phillips and Moon (1999)).

To verify whether the CMLE is asymptotically efficient when $r=1$, we need to compare the asymptotic variance with the CR bound for the conditional model $y_{i, t}=r y_{i, t-1}+\varepsilon_{i, t}$, $\varepsilon_{i, t} \mid y_{i, 1} \sim N\left(0, \sigma^{2}\right)$, (i.i.d.) $t=2, \ldots, T$ and $r=1$. We obtain the following formula for this

\footnotetext{
${ }^{17}$ Andersen (1970) presents a necessary and sufficient condition for equality of the variance of the CMLE to the Cramér-Rao lower bound (see his 5th Theorem). Chamberlain (1980) gives another example of a model where the CMLE does not attain the CR bound, i.e. the logit model. He doubts that a more efficient estimator exists for this model.
} 
CR bound

$$
C R^{C}(1, T)=\frac{2 \sigma^{2}}{(T-1)\left((T-2) \sigma^{2}+2\left(p \lim _{N \rightarrow \infty} \frac{1}{N} \sum_{i=1}^{N} y_{i, 1}^{2}\right)\right)}
$$

assuming that $p \lim _{N \rightarrow \infty} \frac{1}{N} \sum_{i=1}^{N} y_{i, 1}^{2}<\infty$. Notice the dependence of the CR bound on the initial conditions. We find that the asymptotic variance of $\widehat{r}_{C M L}$ is at least four times higher than the CR bound.

The following MLE which is based on the likelihood corresponding to the conditional model given above

$$
\widetilde{r}_{M L}^{C}=\frac{\sum_{i=1}^{N} \sum_{t=2}^{T} y_{i, t} y_{i, t-1}}{\sum_{i=1}^{N} \sum_{t=2}^{T} y_{i, t-1}^{2}}
$$

is not only consistent (when $r=1$ ) but also attains the $\mathrm{CR}$ bound $C R^{C}(1, T)$. However, unlike the CMLE, this estimator is inconsistent for stationary models that contain mean parameters $(1-r) \mu_{i}$. Nevertheless, one may still consider using a unit root test which is based on this statistic.

Various unit root tests for panels with fixed $T$ have already been suggested in the literature. Breitung and Meyer (1994) have proposed an estimator for the ARFE(1) model that, just like the CMLE, is consistent both when $r=1$ and when $|r|<1$. This estimator is based on deviations from the initial observations and has the same large $N$ asymptotic variance as the CMLE. Harris and Tzavalis (1999) present a unit root test statistic for the ARFE(1) model which is an adjusted version of the Least Squares Dummy Variables estimator. The adjustment corrects for the inconsistency of the LSDV estimator. The corrected estimator has asymptotic variance $10.2 /(T-1)(T-2)$.

We will see in section 4 that the CML methodology that has been described above can be applied to any ARMAFE model. Moreover we can generalize the models to allow for individual specific trends and drift parameters respectively. In that case we can obtain consistent estimators by first-differencing the model once more.

Since the CMLE are consistent both in the unit root case and for stationary alternatives, the tests based on the CMLE are consistent. Furthermore, analytical asymptotic results on the power of CMLE based unit tests against local stationary alternatives can more easily be obtained. 


\section{Estimation of dynamic panel data models with initial conditions}

Sometimes we cannot make the stationarity assumption. For instance, when the initial observations $y_{i, 0}, i=1,2, \ldots, N$, are the start-up values of stochastic processes, they may have a distribution that is different from the distribution of the subsequent observations. In that case we may decide to condition on the initial observations, i.e. to treat the initial observations as given. The conditional AR(1) model with fixed effects can be stated as

$$
\begin{aligned}
y_{i} & =r y_{i,-1}+\check{\mu}_{i} \iota+\varepsilon_{i} \\
\check{\mu}_{i} & =(1-r) \mu_{i} \\
\varepsilon_{i} \mid \check{\mu}_{i}, y_{i, 0} & \left.\sim N\left(0, \sigma^{2} I_{T}\right) \text { (i.i.d. }\right)
\end{aligned}
$$

where $i \in\{1,2, \ldots, N\}$. For each individual unit, we have $T+1$ observations on $y$ including the initial observation $y_{i, 0}$.

\subsection{CML and Modified ML estimation of the conditional ARFE(1) model}

In this subsection we study various likelihood based estimators for the conditional ARFE(1) model with $|r|<1$.

We will first study the properties of a CML estimator for this model. To derive this CMLE we need to find sufficient statistics for $\check{\mu}_{i}, i=1,2, \ldots, N$. For this purpose it is helpful to rewrite model (16) in terms of the initial observations. Substituting $y_{i, t}=r y_{i, t-1}+\check{\mu}_{i}+\varepsilon_{i, t}$ repeatedly, we obtain

$$
y_{i}=\lambda_{1} y_{i, 0}+\lambda_{2} \check{\mu}_{i}+P \varepsilon_{i}
$$

where

$$
P=\left[\begin{array}{ccccc}
1 & 0 & \cdot & \cdot & 0 \\
r & 1 & 0 & & \cdot \\
\cdot & r & 1 & 0 & \cdot \\
\cdot & & r & 1 & 0 \\
r^{T-1} & \cdot & \cdot & r & 1
\end{array}\right], \lambda_{1}=\left[\begin{array}{c}
r \\
r^{2} \\
\vdots \\
r^{T-1} \\
r^{T}
\end{array}\right], \text { and } \lambda_{2}=P \iota
$$


Since $\operatorname{det}(P)=1$, the matrix $P$ is nonsingular. We will try to find a vector $b(r)$ such that the conditional distribution $f\left(y_{i} \mid b(r)^{\prime} y_{i}, y_{i, 0}\right)$ does not depend on $\check{\mu}_{i}$. Now

$$
E\left(y_{i} \mid b^{\prime} y_{i}, y_{i, 0}\right)=\lambda_{1} y_{i, 0}+\lambda_{2} \check{\mu}_{i}+\frac{P P^{\prime} b}{b^{\prime} P P^{\prime} b}\left(b^{\prime} y_{i}-b^{\prime} \lambda_{1} y_{i, 0}-b^{\prime} \lambda_{2} \check{\mu}_{i}\right) .
$$

For $\check{\mu}_{i}$ to vanish, $b(r)$ needs to be chosen such that

$$
\frac{P P^{\prime} b}{b^{\prime} P P^{\prime} b} b^{\prime} P \iota-P \iota=0
$$

It is easily verified that $b(r)^{\prime}=\iota^{\prime} P^{-1}$ yields a (minimal) sufficient statistic. The conditional likelihood function given $\iota^{\prime} P^{-1} y_{i}$ is normal with

$$
\begin{aligned}
E\left(y_{i} \mid \iota^{\prime} P^{-1} y_{i}, y_{i, 0}\right) & =\lambda_{1} y_{i, 0}+\frac{1}{T} P \iota\left(\iota^{\prime} P^{-1} y_{i}-\iota^{\prime} P^{-1} \lambda_{1} y_{i, 0}\right) \text { and } \\
\operatorname{Var}\left(y_{i} \mid \iota^{\prime} P^{-1} y_{i}, y_{i, 0}\right) & =\sigma^{2} P Q P^{\prime} .
\end{aligned}
$$

Upon substitution of $y_{i}=\lambda_{1} y_{i, 0}+\lambda_{2} \check{\mu}_{i}+P \varepsilon_{i}$ in $E\left(y_{i} \mid \iota^{\prime} P^{-1} y_{i, 0}\right)$, it follows that $y_{i}-$ $E\left(y_{i} \mid \iota^{\prime} P^{-1} y_{i, 0}\right)=P Q \varepsilon_{i}$. Since $P$ depends on $r$, the conditional likelihood function $l\left(y_{i} \mid \iota^{\prime} P^{-1} y_{i, 0}\right)$ is not proportional to the density of $Q \varepsilon_{i}{ }^{18}$. Therefore, the CML estimator for model (16) does not equal the Within ML estimator. It is well known that the latter is equal to the LSDV estimator and that both are inconsistent. We will see that the CMLE is also inconsistent in this case.

The inconsistency of the CMLE and the Within MLE may be surprising given that the $\check{\mu}_{i}$ are no longer present in the conditional and within likelihood functions. However, we note that the number of initial conditions grows with the cross-sectional dimension of the panel and that they remain important when the time dimension is kept fixed. One may therefore argue that a proper conditional likelihood approach makes use of pairs of sufficient statistics for both the initial conditions $y_{i, 0}$ and the $\check{\mu}_{i}$. These incidental 'parameters' can also be removed from the likelihood function by applying the following transformation matrix to (17): $Z=\left(I-\Lambda\left(\Lambda^{\prime} \Lambda\right)^{-1} \Lambda^{\prime}\right)$, where $\Lambda=\left(\begin{array}{ll}\lambda_{1} & \lambda_{2}\end{array}\right)$. This leads to a tranformed likelihood function with a covariance matrix of rank $T-2$.

\footnotetext{
${ }^{18}$ Notice that these densities only exist in a $T-1$ dimensional subspace. The conditional likelihood function includes the factor $\left|P Q P^{\prime}\right|^{+}$. Also notice that $\left|P Q P^{\prime}\right|^{+} \neq(\operatorname{det}(P))^{2}|Q|^{+}=1$.
} 
Lancaster (1997a,b) obtains a consistent estimator of $r$ and $\sigma^{2}$ by following a Bayesian approach to estimation in the presence of incidental parameters ${ }^{19}$. His approach involves two steps: first, a reparametrization of the model such that the 'new' incidental parameters are information orthogonal to the ('new') common parameters, and second, the new incidental parameters in the likelihood are integrated out using a noninformative, uniform prior.

In the first step he obtains the following reparametrization

$$
\begin{aligned}
\check{\mu}_{i} & =(1-r) y_{i, 0}+\nu_{i} \exp (-\beta(r)) \\
r & =r, \text { and } \sigma^{2}=\sigma^{2}, \\
\text { where } \beta(r) & =\frac{1}{T} \sum_{t=1}^{T-1} \frac{T-t}{t} r^{t}
\end{aligned}
$$

and where $\nu_{i}, i=1,2, \ldots, N$, are the new incidental parameters. Then in the second step he derives the marginal posterior density of $r$ and $\sigma^{2}, p\left(r, \sigma^{2}\right)=\prod_{i=1}^{N} p_{i}\left(r, \sigma^{2}\right)$, as follows

$$
\begin{aligned}
p\left(r, \sigma^{2}\right) \propto & \pi\left(r, \sigma^{2}\right) \prod_{i=1}^{N} \int \sigma^{-T} \exp \left(-\frac{1}{2 \sigma^{2}} \sum_{t=1}^{T}\left(y_{i, t}-r y_{i, t-1}-\check{\mu}_{i}\right)^{2}\right) d \nu_{i} \\
= & \pi\left(r, \sigma^{2}\right) \exp (N \beta(r)) \prod_{i=1}^{N} \int \sigma^{-T} \exp \left(-\frac{1}{2 \sigma^{2}} \sum_{t=1}^{T}\left(y_{i, t}-r y_{i, t-1}-\check{\mu}_{i}\right)^{2}\right) d \check{\mu}_{i} \\
= & \pi\left(r, \sigma^{2}\right) \exp (N \beta(r)) \times \\
& \sigma^{-N(T-1)} \prod_{i=1}^{N} \exp \left(-\frac{1}{2 \sigma^{2}} \sum_{t=1}^{T}\left(y_{i, t}-\bar{y}_{i}-r\left(y_{i, t-1}-\bar{y}_{i,-1}\right)\right)^{2}\right)
\end{aligned}
$$

where $\pi\left(r, \sigma^{2}\right)$ is a noninformative prior for $r$ and $\sigma^{2}$. Notice that in the prior distribution the new incidental parameters and the common parameters are independent. The mode of $p\left(r, \sigma^{2}\right)$ is Lancaster's estimator for $r$ and $\sigma^{2}$.

Theorem 4 A maximum of (23) on $(-1,1) \times(0, \infty)$ is a consistent estimator for $r$ and $\sigma^{2}$ in model (16) with $\left(r, \sigma^{2}\right)$ belonging to a compact subset of $(-1,1) \times(0, \infty)$.

\section{PROOF}

See Appendix B. We also indicate how to select the maximum that is consistent in case there would be multiple maxima.

\footnotetext{
${ }^{19}$ He shows that the derivatives of the expected modified log-likelihood function are zero at the true values of the parameters. For a complete consistency proof see below and Appendix B.
} 
When $N \rightarrow \infty$ the effect of $\pi\left(r, \sigma^{2}\right)$ on $p\left(r, \sigma^{2}\right)$ becomes negligible. However, even though the prior is a so-called noninformative prior, it does add information about the incidental parameters because $T$ is finite.

The marginal posterior density is equal to the Within likelihood function times the factor $\exp (N \beta(r))$. The presence of this factor leads to the consistency of the estimator. If one would follow the modified likelihood approach of Neyman and Scott (1948) starting with the ordinary likelihood function, one would find that the likelihood equations for $\sigma^{2}$ and $r$ have to be modified by adding $\frac{N}{2 \sigma^{2}}$ and $N \beta^{\prime}(r)$, respectively, in order to obtain a consistent estimator for $\sigma^{2}$ and $r$.

Since the conditional likelihood function $l\left(y_{i} \mid \iota^{\prime} P^{-1} y_{i, 0}\right)$ does not contain the factor $\exp (N \beta(r))$, we conclude that the corresponding CMLE is inconsistent in this case. CML is still inconsistent after reparametrization of the model.

The Bayesian approach to estimation in the presence of incidental parameters was already advocated by Lindley and El-Sayyed (1968). They stressed that the prior distribution has to be carefully selected. In particular they mentioned that the use of improper priors can lead to inconsistent estimates in some problems, but can be of considerable use in problems with a group structure, like the problem discussed here. Indeed, in this case the Bayesian approach with improper prior yields consistent estimators.

We will now prove that Lancaster's procedure is equivalent to integrating the incidental parameters out of the model with the original parametrization with respect to Jeffrey's invariant noninformative prior $\operatorname{det}(I M)^{0.5}$, where $I M$ stands for the Information Matrix. Let $L(y, \phi)$ denote the likelihood function, let $\phi$ be the old parameter vector (including the $\check{\mu}_{i}^{\prime} s$ ) and let $\eta$ be the new parameter vector (including the $\nu_{i}^{\prime} s$ ) chosen such that $I M_{\eta}$ is diagonal (the parameters in $\eta$ are information orthogonal). Moreover let $J=\frac{d \eta}{d \phi^{\prime}}$. Now, if the prior $\pi(\eta)$ is chosen equal to $\operatorname{det}\left(I M_{\eta}\right)^{0.5}$, which is a noninformative, 'uniform' prior, then

$$
\begin{aligned}
& \int \ldots \int L(y \mid \phi) \pi(\eta)\left(\prod_{i=1}^{N} \frac{d \nu_{i}}{d \check{\mu}_{i}}\right) d \check{\mu}_{1} \ldots d \check{\mu}_{N}= \\
& \int \ldots \int L(y \mid \phi) \pi(\eta) d \nu_{1} \ldots d \nu_{N}= \\
& \int \ldots \int L(y \mid \phi) \operatorname{det}\left(I M_{\eta}\right)^{0.5} d \nu_{1} \ldots d \nu_{N}=
\end{aligned}
$$




$$
\begin{aligned}
& \int \ldots \int L(y \mid \phi) \operatorname{det}\left(I M_{\eta}\right)^{0.5} \operatorname{det}(J)\left(\prod_{i=1}^{N}\left(\frac{d \nu_{i}}{d \check{\mu}_{i}}\right)^{-1}\right) d \nu_{1} \ldots d \nu_{N}= \\
& \int \ldots \int L(y \mid \phi) \operatorname{det}\left(I M_{\eta}\right)^{0.5} \operatorname{det}(J) d \check{\mu}_{1} \ldots d \check{\mu}_{N}= \\
& \int \ldots \int L(y \mid \phi) \operatorname{det}\left(I M_{\phi}\right)^{0.5} d \check{\mu}_{1} \ldots d \check{\mu}_{N}
\end{aligned}
$$

where the first line in (24) corresponds to (22). The fourth line makes use of $\operatorname{det}(J)=$ $\prod_{i=1}^{N} \frac{d \nu_{i}}{d \breve{\mu}_{i}}=\exp (N \beta(r))$ and the last line follows from the invariance property of Jeffrey's prior (see also Zellner (1971) p. 47). Although Lancaster's reparametrization approach yields exactly the same marginal posterior distribution and estimator as the approach proposed by Lindley and El-Sayyed (1968) which uses $\operatorname{det}\left(I M_{\phi}\right)^{0.5}$, in the case at hand Lancaster's approach is more convenient since $\operatorname{det}\left(I M_{\phi}\right)^{0.5}$ is quite a complicated function ${ }^{20}$. However, application of the modified likelihood approach would have lead to the same estimator for $\sigma^{2}$ and $r$ almost immediately.

To derive the large sample distribution of Lancaster's Modified Maximum Likelihood Estimator one can proceed as if it were an ordinary ML estimator and Taylor expand the modified likelihood equations ${ }^{21}$. However, since the Expected Modified Hessian does not equal minus the Modified Information Matrix (the expected outer product of the modified gradients), one needs to use a 'sandwich' formula for the covariance matrix

$$
\sqrt{N}\left(\begin{array}{c}
\widehat{r}_{C M L}-r \\
\widehat{\sigma}_{C M L}^{2}-\sigma^{2}
\end{array}\right) \rightarrow N\left(0,(E M H)^{-1} M I M(E M H)^{-1}\right)
$$

Both the expected Hessian and the expected outer product of the gradient of the 'modified log-likelihood function' - which is given by (23) apart from the factor $\pi\left(r, \sigma^{2}\right)$ - are easily derived.

\footnotetext{
${ }^{20}$ It is interesting to note that if Lancaster would have used a noninformative, uniform prior in conjunction with the likelihood function with the old parametrization, he would have obtained the Within Likelihood function which yields an inconsistent estimator. This illustrates Lindley and El-Sayyed's point that the prior has to be selected carefully and also shows that one cannot assume that a posterior mode 'always' converges to the true parameter values.

${ }^{21}$ The asymptotic distribution of the MMLE has been obtained independently by Lancaster (1997b).
} 
Let us define

$$
\check{P}=\left(\begin{array}{cccccc}
0 & . & . & 0 & 0 & 0 \\
1 & 0 & & & 0 & 0 \\
r & 1 & 0 & & & 0 \\
\cdot & r & 1 & 0 & & \cdot \\
. & & r & 1 & 0 & . \\
r^{T-2} & . & . & r & 1 & 0
\end{array}\right), \check{\lambda}_{1}=\left(\begin{array}{c}
1 \\
r \\
r^{2} \\
\vdots \\
r^{T-2} \\
r^{T-1}
\end{array}\right)
$$

then

$$
y_{i,-1}=\check{\lambda}_{1} y_{i, 0}+\check{\lambda}_{2} \check{\mu}_{i}+\check{P} \varepsilon_{i}
$$

where $\check{\lambda}_{2}=\check{P} \iota$. The expected modified Hessian equals

$$
E M H=\lim _{N \rightarrow \infty}\left(\begin{array}{cc}
\beta^{\prime \prime}(r)-\frac{1}{N} \sum_{i=1}^{N} \frac{E\left(y_{i,-1}^{\prime} Q y_{i,-1}\right)}{\sigma^{2}} & \frac{\beta^{\prime}(r)}{\sigma^{2}} \\
\frac{\beta^{\prime}(r)}{\sigma^{2}} & -\frac{T-1}{2 \sigma^{4}}
\end{array}\right)
$$

and the modified information matrix equals

$$
M I M=\lim _{N \rightarrow \infty}\left(\begin{array}{cc}
\operatorname{tr}(Q \check{P} Q \check{P})+\frac{1}{N} \sum_{i=1}^{N} \frac{E\left(y_{i,-1}^{\prime} Q y_{i,-1}\right)}{\sigma^{2}} & -\frac{\beta^{\prime}(r)}{\sigma^{2}} \\
-\frac{\beta^{\prime}(r)}{\sigma^{2}} & \frac{T-1}{2 \sigma^{4}}
\end{array}\right)
$$

where we have used the general result $E\left(\left(\varepsilon_{i}^{\prime} M_{1} \varepsilon_{i}\right)\left(\varepsilon_{i}^{\prime} M_{2} \varepsilon_{i}\right)\right)=\sigma^{4}\left(\operatorname{tr}\left(M_{1}\right) \operatorname{tr}\left(M_{2}\right)+\operatorname{tr}\left(M_{1} M_{2}+\right.\right.$ $\left.M_{1}^{\prime} M_{2}\right)$ ) (see e.g. Kiviet (1995)), and $E\left(\varepsilon_{i}^{\prime} Q y_{i,-1}\right)=\sigma^{2} \operatorname{tr}(Q \check{P})=-\sigma^{2} \beta^{\prime}(r)$ (see Lancaster(1997a)). An explicit expression for $\operatorname{tr}(Q \check{P} Q \check{P})$ can be found using Maple. It can easily be checked that $\operatorname{tr}(Q \check{P} Q \check{P}) \neq-\beta^{\prime \prime}(r)$ and therefore $E M H \neq-M I M$. Finally, notice that $E\left(y_{i,-1}^{\prime} Q y_{i,-1}\right)$ depends on $y_{i, 0}$ and $\check{\mu}_{i}$. Using that $E\left(y_{i,-1}^{\prime} Q y_{i,-1}\right) \geq \bar{\sigma}^{2} \operatorname{tr}\left(\check{P}(\bar{r})^{\prime} Q \check{P}(\bar{r})\right)$, it can be shown that the $E M H$ is negative definite symmetric for $-1<r<1^{22}$. A convenient estimator for $\frac{1}{N} \sum_{i=1}^{N} \frac{E\left(y_{i,-1}^{\prime} Q y_{i,-1}\right)}{\sigma^{2}}$ is $\frac{1}{N} \sum_{i=1}^{N} \frac{y_{i,-1}^{\prime} Q y_{i,-1}}{\sigma^{2}}$.

The Cramér-Rao efficiency lowerbound is equal to minus the expected Hessian of the ordinary log-likelihood function. Because the modified log-likelihood function differs from the latter due to the terms $\beta(r)$ and $\log \sigma$, the MMLE for the conditional ARFE(1) model does not attain the $\mathrm{CR}$ lowerbound for this model, $C R^{C}(r, T)$, when $T$ is fixed. Again, the question arises whether there exists an estimator that is more efficient than the consistent ML-type estimator.

\footnotetext{
${ }^{22}$ When $r=1$, the $\check{\mu}_{i}$ disappear and EMH is singular: ignoring the powers of $\sigma$ the diagonal elements of EMH equal $\frac{1}{2}(T-1)$ and the off-diagonal elements of EMH equal $-\frac{1}{2}(T-1)$ when $r=1$.
} 


\subsection{GMM estimation of the conditional ARFE(1) model}

In the previous subsection we assumed that $|r|<1$ and it was convenient to write the conditional model as $y_{i, t}=r y_{i, t-1}+\check{\mu}_{i}+\varepsilon_{i, t}$. However, in the unit root case the individual effects disappear. Using $\check{\mu}_{i}=(1-r) \mu_{i}$, we can rewrite the model as

$$
y_{i, t}=r y_{i, t-1}+(1-r) \mu_{i}+\varepsilon_{i, t}
$$

We now relax the normality assumption $\varepsilon_{i} \mid \check{\mu}_{i}, y_{i, 0} \sim N\left(0, \sigma^{2} I_{T}\right)(i . i . d$.$) and replace it by { }^{23}$

$$
\varepsilon_{i} \sim\left(0, \sigma^{2} I_{T}\right)(\text { i.i.d. }) ; \quad E\left(\check{\mu}_{i} \varepsilon_{i}\right)=0 ; \quad E\left(y_{i, 0} \varepsilon_{i}\right)=0
$$

Then the conditional ARFE(1) model still implies the following restrictions on the second moments (cf Ahn and Schmidt, 1997) ${ }^{24}$ :

$$
\begin{aligned}
E\left(m_{1, t}(r)\right) & =0, t=2, \ldots, T, \\
E\left(m_{2, t}(r)\right) & =0, t=2, \ldots, T, \quad \text { and } \\
E\left(m_{3, s t}(r)\right) & =0,1 \leq s<t, \quad t=3, \ldots, T
\end{aligned}
$$

where

$$
\begin{aligned}
m_{1, t}(r) & =y_{i, 0} \Delta \varepsilon_{i, t} \\
m_{2, t}(r) & =\left(y_{i, t}-r y_{i, t-1}\right)^{2}-\left(y_{i, 1}-r y_{i, 0}\right)^{2} \\
m_{3, s t}(r) & =\left(y_{i, t}-r y_{i, t-1}\right)\left(y_{i, s}-r y_{i, s-1}\right)-\left(y_{i, 2}-r y_{i, 1}\right)\left(y_{i, 1}-r y_{i, 0}\right)
\end{aligned}
$$

The moment conditions in (32) exhaust all the restrictions on the second moments that are implied by the conditional ARFE(1) model.

We define the Optimal Conditional GMM (OCGMM) estimator as the Optimal GMM estimator that exploits the moment conditions in (32).

In addition to the moment conditions in (32), the conditional ARFE(1) model implies the following restrictions on the first moments ${ }^{25}$

$$
E\left(\Delta y_{i, t}-r \Delta y_{i, t-1}\right)=0 \quad t=2, \ldots, T
$$

${ }^{23}$ The results on GMM estimators given below still hold under $\varepsilon_{i} \sim\left(0, \sigma_{i}^{2} I_{T}\right)$ (indep. dist.); $E\left(\check{\mu}_{i} \varepsilon_{i}\right)=0 ; E\left(y_{i, 0} \varepsilon_{i}\right)=0$ as long as the moment conditions do not involve $\sigma_{i}^{2}$.

${ }^{24}$ We notice that assumptions (A.1)-(A.4) in Ahn and Schmidt (1997) are satisfied by this (weaker) version of the conditional $\operatorname{ARFE}(1)$ model.

${ }^{25}$ Crépon et al. (1997) discusses first moment conditions for the AREC(1) model. 
We define the Optimal Best Conditional GMM estimator as the Optimal GMM estimator that exploits the moment conditions in (32) and (33).

Under normality the OBCGMM is the most efficient estimator for $r$ in the conditional ARFE(1) model, because the model is linear and therefore all the information is summarized in the first and second moments of the data.

Under the assumption of cross-sectional homoskedasticity it can be shown along the lines of Ahn and Schmidt (1995) (cf Newey, 1990) that the OCGMM estimator for $r$ attains the semiparametric efficiency bound $S P B^{C}(r, T)$ of the model that is characterized by (32).

The OCGMM estimator is at least as efficient as the MMLE because the latter estimator can be reinterpreted as a GMM estimator that only exploits information in the second moments of the data (see also (62) and (63) in appendix B). In general a MMLE is not always the most efficient estimator for a model even if the underlying distributional assumption is correct. The following result shows that the MMLE for the conditional ARFE(1) model can be strictly less efficient than the OCGMM.

Theorem 5 For $T=2$, the OCGMM estimator for the autoregressive parameter in the conditional ARFE(1) model is more efficient than the MMLE when the data are generated by stationary ARFE(1) models with normally distributed errors.

\section{PROOF}

See Appendix B.

Thus when $T$ is finite and when the data are generated by conditional ARFE(1) models with normally distributed errors and $|r|<1$, we have $\operatorname{Avar}\left(\widehat{r}_{M M L}\right) \geq \operatorname{Avar}\left(\widehat{r}_{O C G M M}\right)=$ $S P B^{C}(r, T) \geq C R^{C}(r, T)$, where Avar denotes large $N$, fixed $T$ asymptotic variance. Under normality and with $|r|<1$, the initial conditions become unimportant when not only $N \rightarrow \infty$ but $T \rightarrow \infty$ as well, and

$$
\begin{aligned}
\lim _{T \rightarrow \infty} \operatorname{Avar}\left(\widehat{r}_{M M L}\right) & =\lim _{T \rightarrow \infty} \operatorname{Avar}\left(\widehat{r}_{O C G M M}\right)=\lim _{T \rightarrow \infty} S P B^{C}(r, T)= \\
\lim _{T \rightarrow \infty} C R^{C}(r, T) & =\lim _{T \rightarrow \infty} C R(r, T)=1-r^{2}
\end{aligned}
$$

To see why these equalities hold asymptotically let us investigate the modified likelihood 
function $\widetilde{p}\left(r, \sigma^{2}\right)$ which is given by (23) apart from the factor $\pi\left(r, \sigma^{2}\right)$

$$
\begin{aligned}
\frac{1}{T} \log \widetilde{p}\left(r, \sigma^{2}\right)= & \frac{\beta(r)}{T}-\frac{(T-1)}{T} \log \sigma \\
& -\frac{1}{2 \sigma^{2}} \frac{1}{N T} \sum_{i=1}^{N} \sum_{t=1}^{T}\left(y_{i, t}-\bar{y}_{i}-r\left(y_{i, t-1}-\bar{y}_{i,-1}\right)\right)^{2}
\end{aligned}
$$

We can easily show that $\lim _{T \rightarrow \infty} \frac{1}{T} \beta(r)=0$. This follows from $|\beta(r)| \leq \frac{1}{T} \sum_{t=1}^{T-1} \frac{T-t}{t}=\sum_{t=1}^{T} \frac{1}{t}-1$ when $|r| \leq 1, \lim _{T \rightarrow \infty}\left(\sum_{t=1}^{T} \frac{1}{t}-\ln T\right) \approx 0.57722$ and from $\lim _{T \rightarrow \infty} \frac{1}{T} \ln T=0$. Therefore,

$$
\lim _{T \rightarrow \infty} \frac{1}{T} \log \widetilde{p}\left(r, \sigma^{2}\right)=-\log \sigma-\lim _{T \rightarrow \infty} \frac{1}{2 \sigma^{2}} \frac{1}{N T} \sum_{i=1}^{N} \sum_{t=1}^{T}\left(y_{i, t}-\bar{y}_{i}-r\left(y_{i, t-1}-\bar{y}_{i,-1}\right)\right)^{2}
$$

We find that when $T \rightarrow \infty$ the modified log-likelihood function converges to the Within log-likelihood function, which is equal to the concentrated log-likelihood function that conditions on the initial observations and that is concentrated with respect to the $\mu_{i}$. When $|r|<1$, the latter converges to the concentrated unconditional log-likelihood function when $T \rightarrow \infty$ since the initial conditions become unimportant.

The equality in (34) implies that under normality and when $|r|<1$ the Optimal Conditional GMM estimator for the conditional model is asymptotically equivalent to the Optimal Linear GMM estimator for the stationary model proposed by Kruiniger (2000) that is defined in section 4.2 when not only $N \rightarrow \infty$ but $T \rightarrow \infty$ as well. The latter exploits two extra second moment conditions that correspond to the initial observations (see Ahn and Schmidt (1997) and section $4.2^{26}$ ). However, when $T \rightarrow \infty$ the initial observations become unimportant and the two extra moment conditions become asymptotically redundant (see section 4.3 for a definition of asymptotic redundancy).

\footnotetext{
${ }^{26}$ Two extra second moment conditions that are implied by stationarity are $E\left(\left(y_{i, 2}-r y_{i, 1}\right) \Delta y_{i, 1}\right)=$ 0 and $E\left((r-1)\left(\Delta y_{i, 1}\right)^{2}-2 \Delta y_{i, 1} \Delta y_{i, 2}\right)=0$.
} 


\section{A comparison of CML, Minimum Distance, and GMM estimators for the closure of the stationary $\operatorname{ARMAFE}(p, q)$ model}

In this section the CMLE (or equivalently the FDMLE) for the closure of the stationary $\operatorname{ARMAFE}(\mathrm{p}, \mathrm{q})$ model, i.e. the inclusive $\operatorname{ARMAFE}(\mathrm{p}, \mathrm{q})$ model, will be compared with GMM estimators in terms of asymptotic efficiency. A direct comparison turns out to be impossible. In order to facilitate the comparison we will introduce a Minimum Distance Estimator (MDE) for the inclusive model that is asymptotically equivalent to the CMLE under normality. This MDE can be reinterpreted as a GMM estimator, which in turn can easily be compared with the GMM estimators from the literature, e.g. the Optimal Linear GMM estimator of Kruiniger (2000). Furthermore, we will compare the large $N$ asymptotic variances of these estimators with the semiparametric and the Cramér-Rao efficiency bounds both for the fixed $T$ case and for the case where $T$ tends to infinity as well, and establish the asymptotic redundancy of the moment conditions involving levels relative to the moment conditions exploited by the (Optimal) MDE.

\subsection{Conditional ML and Minimum Distance estimation}

In this section we will consider estimation of the more general ARMAFE model where (1) is replaced by

$$
\begin{aligned}
A(L)\left(y_{i, t}-\mu_{i}\right) & =B(L) \varepsilon_{i, t} \\
\check{\mu}_{i} & =A(1) \mu_{i} \\
\varepsilon_{i, t} \mid \check{\mu}_{i}, y_{i, t_{0}}, \ldots, y_{i, t_{0}+p-1} & \sim N\left(0, \sigma^{2}\right) \quad(i . i . d .), \quad t=t_{0}+p, \ldots, T \\
t_{0} & \leq-p+2
\end{aligned}
$$

where $A(L)=1-\sum_{k=1}^{p} r_{k} L^{k}, B(L)=1+\sum_{k=1}^{q} r_{p+k} L^{k}$.

Let $d$ denote the order of integration of the processes $\left\{y_{i, t}\right\}$, then we can decompose $A(L)$ as $A(L)=(1-L)^{d} \widetilde{A}(L)$.

We will now define the inclusive $\operatorname{ARMAFE}(p, q)$ model. 
Definition 2 The inclusive ARMAFE(p,q) model is given by (37) and the following assumption

INCL: $\quad d \in\{0,1\}$;

$$
\begin{aligned}
& \left\{(1-L)^{d} y_{i, t}\right\} \text { is a stationary process; } \\
& \left|y_{i, t_{0}}\right|<\infty \text { if } d=1
\end{aligned}
$$

The assumption on the maximal order of integration $(d)$ can easily be relaxed. Assumption INCL allows us to rewrite (37) as

$$
\widetilde{A}(L) D\left(y_{i, t}-\mu_{i}\right)=B(L)(1-L)^{1-d} \varepsilon_{i, t}
$$

This in turn leads to

$$
D\left(y_{i}-\mu_{i} \iota\right)=D y_{i}=D u_{i} \sim N\left(0, \sigma^{2} D V D^{\prime}\right) i . i . d
$$

where $V(=V(r))$ is now a $(T \times T)$ covariance matrix of $\operatorname{ARMA}(\mathrm{p}, \mathrm{q})$ errors (cf. (2) and (3)). See e.g. Van der Leeuw (1994) (henceforth VdL) for the structure of such a covariance matrix. $r$ is an $p+q$-vector comprising the ARMA parameters. When $A(L)$ has a unit root (i.e. $d=1)$, then $\left\{D y_{i, t}\right\}$ will be an $\operatorname{ARMA}(\mathrm{p}-1, \mathrm{q})$ process instead of an $\operatorname{ARMA}(\mathrm{p}, \mathrm{q}+1)$ process. Moreover, when $d=1$, the distibution of $D y_{i}$ is conditional on $y_{i, 1}$, just as in the $\operatorname{ARFE}(1)$ model (so not conditional on $\left.y_{i, 1}, \ldots, y_{i,-p+2} !\right)$.

Before we present various estimators for the inclusive $\operatorname{ARMAFE}(\mathrm{p}, \mathrm{q})$ model, we will first introduce some additional notation: let $\theta=\left(r^{\prime}, \sigma^{2}\right)^{\prime}, Z_{i}=\left(D y_{i}\right)\left(D y_{i}\right)^{\prime}, Z=\frac{1}{N} \sum_{i=1}^{N} Z_{i}$, $\Omega(\theta)=\Omega=\sigma^{2} D V(r) D^{\prime}$, and let $\bar{\Omega}=E\left(Z_{i}\right)=\Omega(\bar{\theta})=\bar{\sigma}^{2} D V(\bar{r}) D^{\prime}$, where upperbars denote the true values of the parameters. Let $\Theta=\Theta_{r} \times(0, \infty)$ denote the parameter space for $\theta$. Finally, let $r^{S}=1-A(1)$.

It is shown in appendix A that $E\left(D y_{i} y_{i}^{\prime} D^{\prime}\right)$ is left-continuous $\left(r^{S} \uparrow 1\right)$ in every $r \in \Theta_{r}$ with $r^{S}=1$ when INCL holds. In addition, it is shown that the first-difference likelihood function is infinitely many times differentiable in (the neighbourhood of) every $r \in \Theta_{r}$ with $r^{S}=1$, when condition INCL is satisfied.

The CMLE for $\theta$ in the inclusive $\operatorname{ARMAFE}(\mathrm{p}, \mathrm{q})$ model is again equivalent to the FDMLE (see also section 2). Following Magnus (1978), the CMLE for $\theta, \widehat{\theta}_{C M L}$, satisfies the following 
likelihood equations

$$
\begin{aligned}
& \operatorname{tr}\left((\Omega(\theta)-Z) \frac{d \Omega(\theta)^{-1}}{d r_{j}}\right)=0, j=1, \ldots, p+q \\
& \operatorname{tr}\left((\Omega(\theta)-Z) \frac{d \Omega(\theta)^{-1}}{d \sigma^{2}}\right)=0
\end{aligned}
$$

or equivalently

$$
\left[\frac{d v e c \Omega}{d \theta^{\prime}}\right]^{\prime}\left(\Omega^{-1} \otimes \Omega^{-1}\right) \operatorname{vec}(Z-\Omega)=0
$$

where we have used $\frac{d \Omega^{-1}}{d \theta_{j}}=-\Omega^{-1} \frac{d \Omega}{d \theta_{j}} \Omega^{-1}$ and $\operatorname{tr} G W H W=(\operatorname{vec} G)^{\prime}(W \otimes W) v e c H$. These likelihood equations are highly nonlinear in $r$.

The asymptotic properties of $\widehat{\theta}_{C M L}$ are given in the following

Theorem 6 The CMLE for $\theta$ in the inclusive ARMAFE(p,q) model is consistent and its large $N$ (fixed $T$ ) asymptotic distribution is given by

$$
\sqrt{N}\left(\widehat{\theta}_{C M L}-\theta\right) \rightarrow N\left(0,2\left(\left[\left.\frac{d v e c \Omega}{d \theta^{\prime}}\right|_{\bar{\theta}}\right]^{\prime}\left(\bar{\Omega}^{-1} \otimes \bar{\Omega}^{-1}\right)\left[\left.\frac{d v e c \Omega}{d \theta^{\prime}}\right|_{\bar{\theta}}\right]\right)^{-1}\right)
$$

\section{ProOF}

For details see appendix A. We will assume that $\bar{\theta} \in \bar{\Theta}$, where $\bar{\Theta}$ is a compact subset of $\Theta$ (The set $\bar{\Theta}$ still includes parameter vectors that correspond to the unit root case, of course). As has already been observed by MaCurdy (1981a), for identification of all the parameters we need that $T>p+q+1$. Then $D V D^{\prime}$ has a unique $\operatorname{ARMA}(\mathrm{p}, \mathrm{q}+1)$ structure (after deleting common divisors). It follows that $\Omega(\theta) \neq \Omega(\bar{\theta})$ if and only if (iff for short) $\theta \neq \bar{\theta}$ and $T>p+q+1$. Furthermore, $\Omega(\theta)$ is nonsingular and differentiable by INCL and results in VdL. It follows that $\log |\Omega(\theta)|$ and $\Omega(\theta)^{-1}$ are differentiable and $E\left(\sup _{\theta \in \bar{\Theta}} l\left(y_{i} \mid \widetilde{\mu}_{i} ; \theta\right)\right)<\infty$.

The asymptotic distribution of $\widehat{\theta}_{C M L}$ can be obtained by Taylor-expanding (41) around $\bar{\theta}$ and using the second Bartlett identity (or by following Magnus (1978) substituting $D y_{i}$ for his $\varepsilon) \square$.

The asymptotic distribution of the Quasi CMLE, $\widehat{\theta}_{Q C M L}$, is given by

$$
\begin{aligned}
\sqrt{N}\left(\widehat{\theta}_{Q C M L}-\theta\right) & \rightarrow N\left(0, H^{-1} G^{\prime}\left(E\left(Z_{i} \otimes Z_{i}\right)-(v e c \bar{\Omega})(v e c \bar{\Omega})^{\prime}\right) G H^{-1}\right. \\
\text { with } & \\
G & =\frac{1}{2}\left(\bar{\Omega}^{-1} \otimes \bar{\Omega}^{-1}\right)\left[\left.\frac{d v e c \Omega}{d \theta^{\prime}}\right|_{\bar{\theta}}\right] \\
H & \left.=\frac{1}{2}\left(\left[\left.\frac{d v e c \Omega}{d \theta^{\prime}}\right|_{\bar{\theta}}\right]^{\prime}\left(\bar{\Omega}^{-1} \otimes \bar{\Omega}^{-1}\right)\left[\left.\frac{d v e c \Omega}{d \theta^{\prime}}\right|_{\bar{\theta}}\right]\right)^{-1}\right)
\end{aligned}
$$


The (Q)CMLE can be used for testing the unit root hypothesis $r^{S}=1$.

Let us now define the Optimal Minimum Distance Estimator (OMDE) for the inclusive $\operatorname{ARMAFE}(\mathrm{p}, \mathrm{q})$ model as ${ }^{27}$

$$
\begin{aligned}
& \widehat{\theta}_{O M D}=\arg \min _{\theta \in \Theta}[\operatorname{vech}(Z-\Omega(\theta))]^{\prime} \times \\
& \times\left(\frac{1}{N} \sum_{i=1}^{N}\left(\left(\operatorname{vech} Z_{i}\right)\left(\operatorname{vech} Z_{i}\right)^{\prime}\right)-(\operatorname{vech} Z)(\operatorname{vech} Z)^{\prime}\right)^{-1} \operatorname{vech}(Z-\Omega(\theta))
\end{aligned}
$$

where $\left(\frac{1}{N} \sum_{i=1}^{N}\left(\left(\right.\right.\right.$ vech $\left.\left.Z_{i}\right)\left(\text { vech } Z_{i}\right)^{\prime}\right)-($ vech $\left.Z)(\text { vech } Z)^{\prime}\right)$ is a consistent estimator of $E\left(\left(\operatorname{vech}\left(Z_{i}-\bar{\Omega}\right)\right)\left(\operatorname{vech}\left(Z_{i}-\bar{\Omega}\right)\right)^{\prime}\right)$, which is the inverse of the (asymptotically) optimal weighting matrix. See also Chamberlain $(1982,1984)$ for the Minimum Distance method.

The asymptotic properties of the OMDE are given in the following

Theorem 7 The OMDE for $\theta$ in the inclusive $A R M A F E(p, q)$ model is consistent and its large $N$ (fixed $T$ ) asymptotic distribution is given by

$$
\begin{array}{r}
\sqrt{N}\left(\widehat{\theta}_{O M D}-\theta\right) \rightarrow N\left(0, C O V_{O M D}\right) \\
\text { where } \\
C O V_{O M D}=\left(\left[\left.\frac{d v e c h \Omega}{d \theta^{\prime}}\right|_{\bar{\theta}}\right]^{\prime}\left(E\left(\left(\operatorname{vech} Z_{i}\right)\left(v_{e c h} Z_{i}\right)^{\prime}\right)-(\operatorname{vech} \bar{\Omega})\left(v_{e c h} \bar{\Omega}\right)^{\prime}\right)^{-1}\left[\left.\frac{d v e c h \Omega}{d \theta^{\prime}}\right|_{\bar{\theta}}\right]\right)^{-1}
\end{array}
$$

Proof

For details see also appendix A. Identification of $\bar{\theta}$ follows from $\operatorname{plim}_{N \rightarrow \infty} Z=E\left(Z_{i}\right)=\bar{\Omega}$, $\bar{\Omega}=\Omega(\theta)$ iff $\theta=\bar{\theta}$, and the fact that the limit of the weighting matrix is nonsingular; moreover, we can find a compact subset of $\Theta$, say $\bar{\Theta}$; it follows from INCL and results in $\operatorname{VdL}$ that $\Omega(\theta)$ is differentiable (and hence continuous) in every $\theta \in \Theta$ and therefore that $\sup _{\theta \in \bar{\Theta}}\|\Omega(\theta)\|<\infty$, where $\|$.$\| is the Euclidean matrix norm. (For example: in the ARFE(1)$ case, $r=-1$ is ruled out by assumption INCL, and $\left.\Omega\left(r=1, \sigma^{2}\right)=\sigma^{2} I\right)$. This together implies consistency of $\widehat{\theta}_{O M D}$ (see Newey and McFadden (1994)).

The asymptotic distribution can easily be obtained by performing a Taylor expansion around the first order condition corresponding to the minimization problem in (44)

${ }^{27}$ Abowd and Card (1989) briefly discuss this estimator in their appendix. 
The OMDE can be reinterpreted as a GMM estimator which is based on the moment conditions in $E(\operatorname{vech}(Z-\Omega(\theta))=0$. The importance of the following theorem is that it permits a comparison between the CMLE and GMM estimators.

Theorem 8 Under normality the CMLE for the inclusive ARMAFE(p,q) model, $\widehat{\theta}_{C M L}$, is asymptotically equivalent to the OMDE, $\widehat{\theta}_{O M D}$.

\section{PROOF}

Let $\check{D}_{T-1}(=\check{D})$ denote the duplication matrix corresponding to a square matrix of dimension $T-1$, let $\check{D}_{T-1}^{+}=\left(\check{D}^{\prime} \check{D}\right)^{-1} \check{D}^{\prime}$ (the Moore-Penrose inverse of $\check{D}$ ), and let $P_{T-1}$ be the commutation matrix (see Magnus and Neudecker (1988)). Then we have under normality $E\left(\left(\operatorname{vec} Z_{i}\right)\left(\operatorname{vec} Z_{i}\right)^{\prime}\right)-(\operatorname{vec} \bar{\Omega})(\operatorname{vec} \bar{\Omega})^{\prime}=(\bar{\Omega} \otimes \bar{\Omega})\left(I_{(T-1)^{2}}+P_{T-1}\right)$. Using that $P_{T-1} \check{D}_{T-1}=\check{D}_{T-1}$, we obtain $C O V_{O M D}^{-1}=\left[\left.\frac{d v e c h \Omega}{d \theta^{\prime}}\right|_{\bar{\theta}}\right]^{\prime}\left(E\left(\left(\text { vech } Z_{i}\right)\left(\text { vech } Z_{i}\right)^{\prime}\right)-(\text { vech } \bar{\Omega})(\operatorname{vech} \bar{\Omega})^{\prime}\right)^{-1}\left[\left.\frac{d v e c h \Omega}{d \theta^{\prime}}\right|_{\bar{\theta}}\right]=$ $\left[\left.\frac{d v e c h \Omega}{d \theta^{\prime}}\right|_{\bar{\theta}}\right]^{\prime}\left(\check{D}_{T-1}^{+}(\bar{\Omega} \otimes \bar{\Omega})\left(I_{(T-1)^{2}}+P_{T-1}\right)\left(\check{D}_{T-1}^{+}\right)^{\prime}\right)^{-1}\left[\left.\frac{d v e c h \Omega}{d \theta^{\prime}}\right|_{\bar{\theta}}\right]=\frac{1}{2}\left[\left.\frac{d v e c h \Omega}{d \theta^{\prime}}\right|_{\bar{\theta}}\right]^{\prime}\left(\check{D}^{+}(\bar{\Omega} \otimes \bar{\Omega})\left(\check{D}^{+}\right)^{\prime}\right)^{-1} \times$ $\left[\left.\frac{d v e c h \Omega}{d \theta^{\prime}}\right|_{\bar{\theta}}\right]=\frac{1}{2}\left[\left.\frac{d v e c h \Omega}{d \theta^{\prime}}\right|_{\bar{\theta}}\right]^{\prime}\left(\check{D}^{\prime} \check{D}\right)\left(\check{D}^{\prime}(\bar{\Omega} \otimes \bar{\Omega}) \check{D}\right)^{-1}\left(\check{D}^{\prime} \check{D}\right) \check{D}+\left[\left.\frac{d v e c \Omega}{d \theta^{\prime}}\right|_{\bar{\theta}}\right]=$ $-\frac{1}{2}\left[\left.\frac{d v e c h \Omega}{d \theta^{\prime}}\right|_{\bar{\theta}}\right]^{\prime}\left(\check{D}^{\prime} \check{D}\right)\left(\check{D}^{\prime}(\bar{\Omega} \otimes \bar{\Omega}) \check{D}\right)^{-1} \check{D}^{\prime}(\bar{\Omega} \otimes \bar{\Omega})\left[\left.\frac{d v e c \Omega^{-1}}{d \theta^{\prime}}\right|_{\bar{\theta}}\right]=$ $-\frac{1}{2}\left[\left.\frac{d v e c h \Omega}{d \theta^{\prime}}\right|_{\bar{\theta}}\right]^{\prime}\left(\check{D}^{\prime} \check{D}\right)\left(\check{D}^{\prime}(\bar{\Omega} \otimes \bar{\Omega}) \check{D}\right)^{-1} \check{D}^{\prime}(\bar{\Omega} \otimes \bar{\Omega}) \check{D}\left[\left.\frac{d v e c h \Omega^{-1}}{d \theta^{\prime}}\right|_{\bar{\theta}}\right]=$ $-\frac{1}{2}\left[\left.\frac{d v e c h \Omega}{d \theta^{\prime}}\right|_{\bar{\theta}}\right]^{\prime}\left(\check{D^{\prime}} \check{D}\right)\left[\left.\frac{d v e c h \Omega^{-1}}{d \theta^{\prime}}\right|_{\bar{\theta}}\right]=\frac{1}{2}\left[\left.\frac{d v e c \Omega}{d \theta^{\prime}}\right|_{\bar{\theta}}\right]^{\prime}\left(\bar{\Omega}^{-1} \otimes \bar{\Omega}^{-1}\right)\left[\left.\frac{d v e c \Omega}{d \theta^{\prime}}\right|_{\bar{\theta}}\right]=C O V_{C M L}^{-1} \square$.

The OMDE has a number of advantages compared to the CMLE. First the OMDE and the corresponding estimator of its asymptotic covariance matrix can more easily be calculated than their CML equivalents. Nevertheless, analytic expressions for the asymptotic variance of the CMLE, like the one that has been derived in section 2 for the $\operatorname{ARFE}(1)$ case, remain useful. For instance, they allow us to derive the asymptotic variance of the CMLE when the model has a unit root. Secondly, the OMDE is more efficient under non-normality (cf MaCurdy (1981b) for the inefficiency of quasi-maximum likelihood estimators of the parameters of a covariance matrix). Thirdly, Chamberlain (1984) has pointed out that the minimum distance method provides a convenient framework for testing restrictions. If the restrictions imposed on $\Omega$ are correct, then

$$
\begin{aligned}
\min _{\theta \in \Theta} N[\operatorname{vech}(Z-\Omega(\theta))]^{\prime}\left(\frac{1}{N} \sum_{i=1}^{N}\left(\left(\operatorname{vech} Z_{i}\right)\left(\operatorname{vech} Z_{i}\right)^{\prime}\right)-(\operatorname{vech} Z)(\operatorname{vech} Z)^{\prime}\right)^{-1} \times \\
\times \operatorname{vech}(Z-\Omega(\theta)) \sim \chi^{2}(d f)
\end{aligned}
$$


where $d f$ is the number of overidentifying restrictions: $0.5(T-1) T-(p+q+1)$. To select the orders of an ARMA model one can follow a sequential test procedure. If an $\operatorname{ARMA}(\mathrm{p}, \mathrm{q})$ specification is rejected, then one drops restrictions until the value of the test statistic is no longer significant. Similarly, if a specification is not rejected, one can impose additional restrictions and test an ARMA model of lower orders (see also Arellano (1990)). In both cases starting values can easily be found. Finally, unit root tests can be based on the (O)MDE.

\subsection{A more efficient GMM estimator}

It is well known that ML estimators can be interpreted as GMM estimators that exploit the expected score vector for an individual observation as the vector of moment conditions. Likewise, the CMLE for $\theta$ in the inclusive $\operatorname{ARMAFE}(\mathrm{p}, \mathrm{q})$ model is a GMM estimator that exploits the following $p+q+1$ moment conditions

$$
\operatorname{Etr}\left(\left(\Omega-Z_{i}\right) \frac{d \Omega^{-1}}{d \theta_{j}}\right)=0 \quad=0 \quad j=1, \ldots, p+q+1
$$

Notice that these moment conditions are highly nonlinear in the parameter $r$. This makes it difficult to compare the CMLE with GMM estimators for dynamic panel data models from the literature.

In the last subsection we have shown that under normality the CMLE is asymptotically equivalent to a Minimum Distance estimator. These Minimum Distance estimators, which are defined by (44), can also be reinterpreted as GMM estimators. They exploit the moment conditions in

$$
\begin{aligned}
E\left(\operatorname{vech}\left(Z_{i}\right)\right)-\operatorname{vech}(\Omega(\theta)) & =0 \\
\text { or } E\left(\operatorname{vech}\left(\left(D y_{i}\right)\left(D y_{i}\right)^{\prime}-\Omega(\theta)\right)\right) & =0
\end{aligned}
$$

where $\Omega \equiv \sigma^{2} D V(r) D^{\prime}$. Note that the number of moment conditions in (48) increases in $T$, whereas the number of moment conditions in (47) does not depend on $T$. Nevertheless, the corresponding Optimal GMM estimators are asymptotically equivalent under normality. However, the moment conditions in (48) are easier to interpret and easier to compare with moment conditions that are exploited by the GMM estimators that have been proposed in the literature. The moment conditions in (48) summarize all the information in the second moments of the (first-)differenced data. 
In order to investigate how the CMLE compares to the GMM estimators from the literature, we will first review the moment conditions that the latter exploit. The literature focuses on the estimation of the AR(1) model with individual effects. The most basic GMM estimator for this model has been proposed by Arellano and Bond (1991). It exploits the moment conditions which follow from the standard assumption

$$
E\left(\varepsilon_{i, t} \mid y_{i, t-s}\right)=0 \text { for } s=1, \ldots, t-1 \text { and } t=2, \ldots, T
$$

This assumption implies $0.5(T-1)(T-2)$ basic moment conditions

$$
E\left(y_{i, t-s} \Delta \varepsilon_{i, t}\right)=0 \text { for } s=2, \ldots, t-1 \text { and } t=3, \ldots, T
$$

where $\Delta \varepsilon_{i, t}=\varepsilon_{i, t}-\varepsilon_{i, t-1}$. Often one also makes the assumption that the errors are uncorrelated with the individual effects

$$
E\left(\varepsilon_{i, t} \mu_{i}\right)=0 \text { for } s=1, \ldots, t-1 \text { and } t=2, \ldots, T
$$

This assumption gives rise to $T-3$ additional moment conditions (see Ahn and Schmidt (1995))

$$
E\left(\left(y_{i, T}-r y_{i, T-1}\right) \Delta \varepsilon_{i, t-1}\right)=0 \text { for } t=4, \ldots, T
$$

Notice that these moment conditions are non-linear in $r$. An alternative assumption is the following

$$
E\left[\left(y_{i, t}-y_{i, t-1}\right) \mu_{i}\right]=0 \text { for } t=2, \ldots, T
$$

This assumption adds $T-2$ moment conditions to the basic moment conditions, which are also linear in $r$ (see Arellano and Bover (1995))

$$
E\left(\left(y_{i, t}-r y_{i, t-1}\right) \Delta y_{i, t-1}\right)=0 \text { for } t=3, \ldots, T
$$

Using these moment conditions renders (50) redundant for estimation. Finally, adding the assumption of homoskedasticity

$$
E\left(\varepsilon_{i, t}^{2}\right)=\sigma^{2} \text { for } t=2, \ldots, T
$$

implies another T-2 moment conditions (see Ahn and Schmidt (1995)) ${ }^{28}$

$$
E\left(y_{i, t} \varepsilon_{i, t}-y_{i, t-1} \varepsilon_{i, t-1}\right)=0 \text { for } t=3, \ldots, T
$$

\footnotetext{
${ }^{28}$ In fact we only need homoskedasticity in the time dimension $\sigma_{i, t}^{2}=\sigma_{i}^{2} \forall i, t$.
} 
Notice that these moment conditions are also linear in $\mathrm{r}$. Therefore, when all the assumptions listed above hold, a GMM estimator for $r$ can be constructed that exploits a total of $0.5 T \times$ $(T+1)-3$ linear moment conditions.

All the moment conditions (and assumptions) just mentioned are also compatible with the stationary dynamic model with fixed effects, which was stated in section 2 . In fact all the moment conditions given in (48)-(52) still hold under $\varepsilon_{i} \sim\left(0, \sigma^{2} I_{T-1}\right)$ (i.i.d.) ; $E\left(\check{\mu}_{i} \varepsilon_{i}\right)=0$; $E\left(y_{i, 1} \varepsilon_{i}\right)=0$ and covariance stationarity of $\left\{y_{i, t}\right\}^{29}$. However, we have not yet exhausted the set of all the moment conditions that are implied by covariance stationarity of $\left\{y_{i, t}\right\}$. Covariance stationarity of $\left\{y_{i, t}\right\}$ implies a total of $0.5 T(T+1)$ moment conditions corresponding to $E\left(\operatorname{vech}\left(y_{i} y_{i}^{\prime}\right)\right)$, or equivalently $0.5 T(T+1)$ moment conditions corresponding to $E\left[\operatorname{vech}\left(\left(D y_{i}\right)\left(D y_{i}\right)^{\prime}\right)\right], E\left(D y_{i} y_{i, 1}\right)$, and $E\left(y_{i, 1}^{2}\right)^{30}$. Clearly, these moment conditions not only involve $r$ but also $\sigma^{2}$. Moreover, when $|r|<1$, the last moment condition also depends on $\mu_{i}^{2}$. When $r=1$, estimation proceeds by conditioning on $y_{i, 1}$ and the last moment condition disappears altogether. Therefore the last moment condition is not instrumental for estimating $r$. We lose another moment condition when eliminating $\sigma^{2}$. A count of moment conditions suggests that there is still one moment condition missing. Ahn and Schmidt (1997) observed that covariance stationarity of $\left\{y_{i, t}\right\}$ also implies that

$$
E\left(y_{i, 1}^{2}+y_{i, 2} \Delta \varepsilon_{i, 3} /\left(1-r^{2}\right)-\left(y_{i, 3}-r y_{i, 2}\right)\left(y_{i, 2}-r y_{i, 1}\right) /(1-r)^{2}\right)=0
$$

Notice that the last moment condition is non-linear in $r$ and therefore gives rise to a nonlinear GMM estimator.

Kruiniger (2000) shows that there exists a linear GMM estimator for $r$ that exploits all the information in the second moments. This Linear GMM estimator combines (49), (51),

\footnotetext{
${ }^{29}$ The results on GMM estimators given below still hold under $\varepsilon_{i} \sim\left(0, \sigma_{i}^{2} I_{T-1}\right)$ (indep. dist.); $E\left(\check{\mu}_{i} \varepsilon_{i}\right)=0 ; E\left(y_{i, 1} \varepsilon_{i}\right)=0$ and covariance stationarity of $\left\{y_{i, t}\right\}$, as long as the moment conditions do not involve $\sigma_{i}^{2}$.

${ }^{30}$ Covariance stationarity also implies restrictions on the first moments: $E\left(\Delta y_{i, t}\right)=0, t=2, \ldots, T$. Under normality the most efficient estimator for $r$ in the stationary model exploits the moment conditions just mentioned and all the moment conditions that are exploited by the Linear GMM estimator that is defined below. We call this most efficient estimator the Optimal Best Linear GMM (OBLGMM) estimator.
} 
(52), and the linear moment condition

$$
E\left((r-1)\left(\Delta y_{i, 2}\right)^{2}-2 \Delta y_{i, 2} \Delta y_{i, 3}\right)=0
$$

instead of (53).

In section 2 it was observed that the CMLE does not attain the Cramér-Rao lowerbound when $T$ is finite. Since the CMLE only exploits information that is contained in the second moments of the data, the Optimal Linear GMM estimator is at least as efficient as the CMLE. It turns out that under normality the Optimal Linear GMM estimator is strictly more efficient than the CMLE when $T$ is finite. We have the following result:

Theorem 9 Under normality the Optimal Linear GMM estimator for $r$ is more efficient than the Optimal GMM estimator based on $E\left(\operatorname{vech}\left(Z_{i}\right)\right)-\operatorname{vech}\left(\Omega\left(r, \sigma^{2}\right)\right)=0$ when $|r|<1$ and $T=3$.

\section{PROOF}

See appendix C. It is shown that under the assumption of normality the moment condition $E\left(y_{i, 1} \Delta \varepsilon_{i, 3}\right)=0$ is not redundant relative to $E\left(\operatorname{vech}\left(Z_{i}\right)\right)-\operatorname{vech}\left(\Omega\left(r, \sigma^{2}\right)\right)=0$ when $|r|<1$ and $T=3$. The property of redundancy is defined by Breusch et al. (1999). In Kruiniger (1998b, 2000) we consider the case $r=1$.

Above we have noted that under non-normality the OMDE is generally more efficient than the CMLE. We conclude that the Optimal Linear GMM estimator is generally more efficient than the CMLE.

Some of the results that we obtained above can be generalized. A fully efficient GMM estimator for $r$ and $\sigma^{2}$ in the stationary $\operatorname{ARMAFE}(\mathrm{p}, \mathrm{q})$ model (i.e. with $\left|r^{S}\right|<1$ ) is based on the following $0.5 T(T+3)-2$ moment conditions

$$
\begin{aligned}
& E\left[\operatorname{vech}\left(\left(D y_{i}\right)\left(D y_{i}\right)^{\prime}\right)\right]-\sigma^{2} \operatorname{vech}\left(D V(r) D^{\prime}\right)=0 \\
& E\left(D y_{i} y_{i, 1}\right)-\sigma^{2} D V(r) e_{1}=0 \\
& E\left(D y_{i}\right)=0
\end{aligned}
$$

where $e_{1}=\left[\begin{array}{lllll}1 & 0 & 0 & \ldots\end{array}\right]^{\prime}$. For the $\operatorname{ARFE}(1)$ model it is easily verified that $E\left(y_{i, 1} \Delta y_{i, t}\right)=$ $-\frac{r^{t-2} \sigma^{2}}{r+1}$ when $|r|<1$ and $E\left(y_{i, 1} \Delta y_{i, t} \mid y_{i, t_{0}}\right)=0$ when $r=1$. This implies that $E\left(D y_{i} y_{i, 1}\right)$ is 
left-discontinuous at $r=1^{31}$. Therefore $E\left(D y_{i} y_{i, 1}\right)-\sigma^{2} D V e_{1}=0$ as such cannot be used for the estimation of the inclusive $\operatorname{ARFE}(1)$ model. Estimation of the inclusive ARFE(1) model is studied in Kruiniger (2000).

The GMM estimation method has still another advantage relative to the CML approach. Because the GMM approach can exploit information in levels as well, it can be used to estimate more complicated models. For instance, when $T=3$, the moment conditions $E\left(\operatorname{vech}\left(Z_{i}\right)\right)-\operatorname{vech}\left(\Omega\left(r, \sigma^{2}\right)\right)=0$ only permit identification of the parameters of an ARFE(1) model. After adding $E\left(\varepsilon_{i, 3} \Delta y_{i, 1}\right)=0$, the parameters of an $\operatorname{ARFE}(2)$ model can be identified.

\subsection{Efficiency}

Under the assumption of cross-sectional homoskedasticity it can be shown along the lines of Ahn and Schmidt (1995) (cf Newey, 1990) that the OLGMM estimator for $r$ attains the semiparametric efficiency bound $S P B(r, T)$ of a stationary model that is characterized by (49), (51), (52) and (54) (and $|r|<1$ ). These moment conditions exhaust the set of second moment conditions that are implied by the assumption of covariance stationarity. Now, for finite $T, S P B(r, T)$ is strictly larger than the Cramér-Rao bound $C R(r, T)$, because the latter does not depend on the $\mu_{i}$ 's. We have therefore the following ranking when $|r|<1$ and $T$ is finite

$$
\operatorname{Avar}\left(\widehat{r}_{C M L}\right) \geq \operatorname{Avar}\left(\widehat{r}_{O M D E}\right)>\operatorname{Avar}\left(\widehat{r}_{O L G M M}\right)=S P B(r, T)>C R(r, T)
$$

However, with normally distributed errors the CMLE attains the Cramér-Rao lowerbound when $T \rightarrow \infty$. It follows that under normality $\lim _{T \rightarrow \infty} \operatorname{Avar}\left(\widehat{r}_{C M L}\right)=\lim _{T \rightarrow \infty} \operatorname{Avar}\left(\widehat{r}_{O M D E}\right)$ $=\lim _{T \rightarrow \infty} \operatorname{Avar}\left(\widehat{r}_{O L G M M}\right)=\lim _{T \rightarrow \infty} C R(r, T)=1-r^{2}$. This implies that under normality when $T \rightarrow \infty$ some of the moment conditions exploited by the Optimal Linear GMM estimator become redundant in the sense of Breusch et al. (1999) relative to the moment conditions exploited by the OMDE, (48), that is, they are asymptotically redundant relative

\footnotetext{
${ }^{31}$ It can be shown that $E\left(D y_{i} y_{i, 1}\right)$ is left-discontinuous at $r^{S}=1$ for more general inclusive $\operatorname{ARMAFE}(\mathrm{p}, \mathrm{q})$ models as well.
} 
to $(48)^{32}$. We will now give a general formal definition of asymptotic redundancy ${ }^{33}$.

Definition 3 Let a model be given by $E\left(g_{i, T}(\theta)\right)=0, i=1, \ldots, N$, where $g_{i, T}(\theta)=\left(g_{1, i, T}^{\prime}(\theta)\right.$ $\left.g_{2, i, T}^{\prime}(\theta)\right)^{\prime}$ and $\operatorname{dim}\left(g_{i, T}(\theta)\right)$ depends on $T$, and let $O G M M E\left(g_{p, i, T}\right)$ be the Optimal GMM estimator that exploits $E\left(g_{p, i, T}(\theta)\right)=0$. Then $E\left(g_{2, i, T}(\theta)\right)=0$ is asymptotically redundant relative to $E\left(g_{1, i, T}(\theta)\right)=0$ iff $\lim _{T \rightarrow \infty} \operatorname{Avar}\left(O G M M E\left(g_{1, i, T}\right)\right)=\lim _{T \rightarrow \infty} \operatorname{Avar}\left(O G M M E\left(g_{i, T}\right)\right)$, where $\operatorname{Avar}\left(O G M M E\left(g_{p, i, T}\right)\right)$ is the large $N$ asymptotic variance of $O G M M E\left(g_{p, i, T}\right)$.

Asymptotic redundancy can only occur in multi-dimensional models, e.g. panel data models. Let us finally present a necessary and sufficient condition for asymptotic redundancy. For that purpose we introduce some additional notation. Let $G=E\left(\frac{\partial g}{\partial \theta^{\prime}}\right), W=E\left(g g^{\prime}\right)$, $h_{2}=g_{2}-W_{21} W_{11}^{-1} g_{1}, h=\left(g_{1}^{\prime} h_{2}^{\prime}\right)^{\prime}, H=E\left(\frac{\partial h}{\partial \theta^{\prime}}\right)$, and $S=E\left(h h^{\prime}\right)$. Notice that $S_{12}=0$. Then we can state the following result:

Theorem $10 E\left(g_{2}(\theta)\right)=0$ is asymptotically redundant relative to $E\left(g_{1}(\theta)\right)=0$ if and only if $\lim _{T \rightarrow \infty} \frac{\left[H_{2}^{\prime} S_{22}^{-1} H_{2}\right]_{k k}}{\left[G_{1}^{\prime} W_{11}^{-1} G_{1}\right]_{l l}}=0$ for all $k \in\left\{1, \ldots, \operatorname{dim}\left(g_{2}\right)\right\}$ and for an arbitrary $l \in\left\{1, \ldots, \operatorname{dim}\left(g_{1}\right)\right\}$.

\section{PROOF}

Trivial.

\footnotetext{
${ }^{32}$ In fact under normality all moment conditions that differ from the moment conditions that are exploited by the OMDE are asymptotically redundant relative to the latter when $N, T \rightarrow \infty$.

${ }^{33}$ Breusch et al. (1999) do not consider asymptotic redundancy.
} 


\section{Conclusions}

In this paper we have studied Maximum Likelihood estimators for two types of dynamic panel data models with fixed effects: a conditional ARFE(1) model and inclusive ARMAFE models (which includes both the stationary models and the unit root model). We have also compared the consistent ML-type estimators for these models with Generalized Method of Moments estimators.

We have proven that the Conditional ML estimators for the inclusive ARMAFE models are consistent and under normality asymptotically equivalent to the Optimal GMM estimator that exploits the restrictions on the second moments of the differenced data (the OMDE). In this connection we have shown that the conditional likelihood functions for the inclusive ARMAFE models are differentiable at the unit root. We have also suggested CML based unit root tests. For the conditional ARFE(1) model the CML approach that only conditions on sufficient statistics for the individual mean parameters leads to an inconsistent estimator.

For both the the conditional ARFE(1) model and the stationary ARFE(1) model we have shown that the most efficient GMM estimators that only exploit restrictions on the second moments of the data are asymptotically generally more efficient than the MMLE of Lancaster (1997a,b) and the CMLE, respectively, when the time-dimension of the panel is fixed. In particular, we have established that the Optimal Linear GMM estimator of Kruiniger (2000) for the inclusive model is asymptotically more efficient than the OMDE of Chamberlain $(1982,1984)$. However, under normality and when the autoregressive parameter is less than one all these estimators attain the Cramér-Rao lowerbound when not only the cross-sectional dimension but also the time dimension tends to infinity. This implies that some moment conditions, i.e. those involving levels and the moment conditions that are related to the initial conditions, are asymptotically redundant. 


\section{A Asymptotic properties of CML Estimators for INCLUSIVE ARMAFE(P,Q) MODELS.}

Van der Leeuw (1994) is henceforth abbreviated as VdL.

We distinguish the following parameter spaces for $\theta=\left(r^{\prime} \sigma^{2}\right)^{\prime}$ : the parameter space corresponding to the inclusive model, $\Theta=\Theta_{r} \times(0, \infty)$; the set $\Theta_{r}^{U R}=\left\{r \mid r \in \Theta_{r}, r^{S}=1\right\}$; the set $\Theta_{r}^{U R Z}=\left\{r \mid r \in \Theta_{r}^{U R}, r_{p+1}=0, \ldots, r_{q}=0\right\}$; the set $\Theta_{r}^{S}=\left\{r \mid r \in \Theta_{r}, r^{S}<1\right\}$; the compact parameter space $\bar{\Theta}=\bar{\Theta}_{r} \times\left[\delta_{2}, \frac{1}{\delta_{2}}\right]$, where $\delta_{2}>0$ is a small number, and which is such that $\bar{\Theta} \subset \Theta$ and $\Theta_{r}^{U R Z} \subset \bar{\Theta}_{r}$; and the extended parameter space $\Theta^{\delta}=\Theta_{r}^{\delta} \times(0, \infty)$, where $\Theta_{r}^{\delta}$ is an open set and $\Theta_{r} \subset \Theta_{r}^{\delta}$. A more precise definition of $\Theta_{r}^{\delta}$ will be given below.

$\operatorname{VdL}$ has shown that an $\operatorname{ARMA}(\mathrm{p}, \mathrm{q})$ matrix $V$ can be factorized as $V=K W^{-1} K^{\prime}$, where $K$ is linear in the MA parameters, and $W$ consists of second order polynomials of the AR parameters. $W^{-1}$ is an $\operatorname{AR}(\mathrm{p})$ covariance matrix.

The matrix $W$ may be singular for particular values of the vector of AR parameters. Nevertheless, expressions involving $W^{-1}$, e.g. $D W^{-1} D^{\prime}$, may still exist for such values of the vector of AR parameters.

We can factorize the autoregressive lag-polynomial $A(L)$ as $\prod_{k=1}^{p}\left(1-a_{k} L\right)$, where w.l.o.g. $\left|a_{1}\right| \leq 1,\left|a_{i}\right|<1, i=2, \ldots, p$. The unit root case $r^{S}=1$ corresponds to $a_{1}=1$ and stationarity $r^{S}<1$ corresponds to $a_{1}<1$.

Let $a=\left(a_{1} a_{2} \ldots a_{p}\right)^{\prime}, \Theta_{a}=\left\{a|| a_{1}|\leq 1,| a_{i} \mid<1, i=2, \ldots, p\right\}$ and let $\Theta_{a}^{U R}=\left\{a|| a_{1} \mid=1\right.$, $\left.\left|a_{i}\right|<1, i=2, \ldots, p\right\}$.

We will first show that $E\left(D y_{i} y_{i}^{\prime} D^{\prime}\right)$ is left continuous for every $r \in \Theta_{r}^{U R}$, i.e. $E\left(D y_{i} y_{i}^{\prime} D^{\prime}\right)$ is continuous between the stationary cases and the unit root cases and therefore we can use the formula $D V D^{\prime}$ for every $r \in \Theta_{r}^{U R}$ as well. Let $R(r)=D V D^{\prime}$ then we can state the following

Lemma 11 For any $p, q, T<\infty, \lim _{n \rightarrow \infty} R\left(\check{r}_{n}\right)=R(r)$ for every $r \in \Theta_{r}^{U R}$ and for every sequence $\left\{\check{r}_{n}\right\}_{n=1}^{\infty}$ with $\lim _{n \rightarrow \infty} \check{r}_{n}=r$, and $\check{r}_{n} \in \Theta_{r}, \forall n \geq 1$.

\section{PROOF}

We will first prove the lemma for $R=D W^{-1} D^{\prime}$, where $W^{-1}$ is an arbitrary AR(p) covariance matrix. So initially we will assume that $B(L)=I$. 
Observe that we allow for any sequence $\left\{\check{r}_{n}\right\}$ in $\Theta_{r}$ that converges to $r \in \Theta_{r}^{U R}$. Equivalently, we can consider any sequence $\left\{\check{a}_{n}\right\}$ in $\Theta_{a}$ that converges to $a \in \Theta_{a}^{U R}$, since by definition $\forall r \in \Theta_{r}, \exists a \in \Theta_{a}$ such that $1-\sum_{k=1}^{p} r_{k} L^{k}=\prod_{k=1}^{p}\left(1-a_{k} L\right)$, and since $\forall a \in \Theta_{a}$, $\exists$ ! $r \in \Theta_{r}$ such that $1-\sum_{k=1}^{p} r_{k} L^{k}=\prod_{k=1}^{p}\left(1-a_{k} L\right)$.

We decompose $A(L)$ as $\bar{A}(L)\left(1-a_{1} L\right)$. Let's define $\omega_{i, t}=\bar{A}(L)^{-1} B(L) \varepsilon_{i, t}$. Then we can write $A(L)\left(y_{i, t}-\mu_{i}\right)=B(L) \varepsilon_{i, t}$ as $\left(1-a_{1} L\right)\left(y_{i, t}-\mu_{i}\right)=\omega_{i, t}$, and $\Delta y_{i, t}=\sum_{m=0}^{\infty} a_{1}^{m} \omega_{i, t-m}-\sum_{s=0}^{\infty} a_{1}^{m} \omega_{i, t-1-m}=\sum_{m=0}^{\infty} a_{1}^{m}\left(a_{1}-1\right) \omega_{i, t-1-m}+\omega_{i, t}$.

Define for arbitrary $s, t$ and $\left|a_{1}\right| \leq 1 E\left(s, t, a_{1}\right) \equiv$ $E\left[\left(\sum_{m=0}^{\infty} a_{1}^{m}\left(a_{1}-1\right) \omega_{i, s-1-m}+\omega_{i, s}\right)\left(\sum_{m=0}^{\infty} a_{1}^{m}\left(a_{1}-1\right) \omega_{i, t-1-m}+\omega_{i, t}\right)\right]$.

We assume that $t-s$ is finite and w.l.o.g. that $s \leq t$. Let us also define $E\left(s, t, a_{1}, 1\right) \equiv E\left[\left(\sum_{m=0}^{\infty} a_{1}^{m}\left(a_{1}-1\right) \omega_{i, s-1-m}\right)\left(\sum_{m=0}^{\infty} a_{1}^{m}\left(a_{1}-1\right) \omega_{i, t-1-m}\right)\right]$, $E\left(s, t, a_{1}, 2\right) \equiv E\left[\left(\sum_{m=0}^{\infty} a_{1}^{m}\left(a_{1}-1\right) \omega_{i, s-1-m}\right)\left(\omega_{i, t}\right)\right]$, and $E\left(s, t, a_{1}, 3\right) \equiv E\left[\left(\omega_{i, s}\right)\left(\sum_{m=0}^{\infty} a_{1}^{m}\left(a_{1}-1\right) \omega_{i, t-1-m}\right)\right]$. Then $E\left(s, t, a_{1}\right)=E\left(s, t, a_{1}, 1\right)+E\left(s, t, a_{1}, 2\right)+E\left(s, t, a_{1}, 3\right)+E\left(\omega_{i, s} \omega_{i, t}\right)$.

W.l.o.g. we will only consider sequences $\left\{\check{a}_{n}\right\}$ in which only $a_{1}$ changes.

We will show that $\lim _{a_{1} \uparrow 1} E\left(s, t, a_{1}, 1\right)=0, \lim _{a_{1} \uparrow 1} E\left(s, t, a_{1}, 2\right)=0$, and $\lim _{a_{1} \uparrow 1} E\left(s, t, a_{1}, 3\right)=0 \forall s, t$.

For this purpose, we will first examine $E\left[\sum_{m=0}^{\infty} a_{1}^{m} \omega_{i, t-m} \omega_{i, t}\right]$.

Since $\left\{w_{i, t}\right\}$ is a stationary $\operatorname{AR}(\mathrm{p}-1)$ process, $E\left[\sum_{s=0}^{\infty} a_{1}^{s} \omega_{i, t} \omega_{i, t-s}\right]=\left[\sum_{s=0}^{\infty} a_{1}^{s} \rho_{s}\right] E\left(\omega_{i, t}^{2}\right)$, where $\rho_{s}$ is the $s$-th autocorrelation. Stationarity of $\left\{\omega_{i, t}\right\}$ also implies that $E\left(\omega_{i, t}^{2}\right)<$ $\infty$. Moreover, the autocorrelations $\rho_{s}$ satisfy the Yule-Walker equations: $\rho_{s}=\sum_{k=1}^{p-1} \Psi_{k} \lambda_{k}^{s}$, where $\Psi_{k}$ and $\lambda_{k}$ are constants and $\left|\lambda_{k}\right|<1$. Upon substitution, we obtain $\sum_{s=0}^{\infty} a_{1}^{s} \rho_{s}=$ $\sum_{s=0}^{\infty} a_{1}^{s} \sum_{k=1}^{p-1} \Psi_{k} \lambda_{k}^{s}=\sum_{k=1}^{p-1} \Psi_{k} \sum_{s=0}^{\infty} a_{1}^{s} \lambda_{k}^{s}=\sum_{k=1}^{p-1} \frac{\Psi_{k}}{1-a_{1} \lambda_{k}}$ since $\left|a_{1} \lambda_{k}\right|<1, k=1,2, \ldots$, $p-1$.

Let us define $E\left(t, a_{1}\right) \equiv\left(1-a_{1}\right) E\left[\sum_{s=0}^{\infty} a_{1}^{s} \omega_{i, s} \omega_{i, t-s}\right] \forall t$.

We find that $\lim _{a_{1} \uparrow 1} E\left(t, a_{1}\right)=\lim _{a_{1} \uparrow 1} E\left(\omega_{i, t}^{2}\right) \sum_{k=1}^{p-1} \frac{\Psi_{k}\left(1-a_{1}\right)}{1-a_{1} \lambda_{k}}=0 \forall t$.

Let us now consider $E\left(s, t, a_{1}, 1\right)$. $E\left(s, t, a_{1}, 1\right)=\left(a_{1}-1\right)^{2} E\left[\left(\sum_{m=0}^{\infty} a_{1}^{m} \omega_{i, s-1-m}\right)\left(\sum_{m=0}^{\infty} a_{1}^{m} \omega_{i, t-1-m}\right)\right]$.

Now, $E\left[\left(\sum_{m=0}^{\infty} a_{1}^{m} \omega_{i, s-m}\right)\left(\sum_{m=0}^{\infty} a_{1}^{m} \omega_{i, t-m}\right)\right]=$ $E\left(\sum_{m=0}^{t-s-1} a_{1}^{m} \omega_{i, t-m}\right)\left(\sum_{m=0}^{\infty} a_{1}^{m} \omega_{i, s-m}\right)+a_{1}^{t-s} E\left(\sum_{m=0}^{\infty} a_{1}^{m} \omega_{i, s-m}\right)^{2}$, and $E\left(\sum_{m=0}^{\infty} a_{1}^{m} \omega_{i, s-m}\right)^{2}=E\left[-\sum_{m=0}^{\infty} a_{1}^{2 m} \omega_{i, s-m}^{2}+2 \sum_{m=0}^{\infty} \sum_{l=m}^{\infty} a_{1}^{m+l} \omega_{i, s-m} \omega_{i, s-l}\right]=$ 
$E\left[-\sum_{m=0}^{\infty} a_{1}^{2 m} \omega_{i, s-m}^{2}\right]+2 E\left[\omega_{i, s}^{2}\right] \sum_{m=0}^{\infty} a_{1}^{2 m} \sum_{l=m}^{\infty} a_{1}^{l-m} \rho_{i, l-m}=$

$-\frac{1}{1-a_{1}^{2}} E\left[\omega_{i, s}^{2}\right]+\frac{2}{1-a_{1}^{2}} E\left[\omega_{i, s}^{2}\right] \sum_{k=1}^{p-1} \frac{\Psi_{k}}{1-a_{1} \lambda_{k}}$.

Therefore $\lim _{a_{1} \uparrow 1}\left(a_{1}-1\right)^{2} a_{1}^{t-s} E\left(\sum_{m=0}^{\infty} a_{1}^{m} \omega_{i, s-m}\right)^{2}=0$.

$E\left(\sum_{m=0}^{t-s-1} a_{1}^{m} \omega_{i, t-m}\right)\left(\sum_{m=0}^{\infty} a_{1}^{m} \omega_{i, s-m}\right)=$

$E\left(\sum_{k=0}^{t-s-1} a_{1}^{k} \omega_{i, t-k} a_{1}^{s+k-t}\left(\sum_{m=0}^{\infty} a_{1}^{m} \omega_{i, t-k-m}-\sum_{m=0}^{t-k-s-1} a_{1}^{m} \omega_{i, t-k-m}\right)\right)$.

By stationarity of $\left\{\omega_{i, t}\right\}$ there is a constant $C$ (which does not depend on $a_{1}$ ) such that $\left|E\left(\sum_{k=0}^{t-s-1} a_{1}^{k} \omega_{i, t-k} a_{1}^{s+k-t} \sum_{m=0}^{t-k-s-1} a_{1}^{m} \omega_{i, t-k-m}\right)\right|<C$ and therefore

$\lim _{a_{1} \uparrow 1}\left(a_{1}-1\right)^{2} E\left(\sum_{k=0}^{t-s-1} a_{1}^{k} \omega_{i, t-k} a_{1}^{s+k-t} \sum_{m=0}^{t-k-s-1} a_{1}^{m} \omega_{i, t-k-m}\right)=0$.

$E\left(\sum_{k=0}^{t-s-1} a_{1}^{k} \omega_{i, t-k} a_{1}^{s+k-t} \sum_{m=0}^{\infty} a_{1}^{m} \omega_{i, t-k-m}\right)=$

$a_{1}^{s-t} E\left(\sum_{k=0}^{t-s-1} a_{1}^{2 k} \omega_{i, t-k} \sum_{m=0}^{\infty} a_{1}^{m} \omega_{i, t-k-m}\right)=\frac{a_{1}^{s-t}}{1-a_{1}} \sum_{k=0}^{t-s-1} a_{1}^{2 k} E\left(t-k, a_{1}\right)$.

Since $\lim _{a_{1} \uparrow 1} E\left(t, a_{1}\right)=0 \forall t$, we find that

$\lim _{a_{1} \uparrow 1}\left(a_{1}-1\right)^{2} E\left(\sum_{k=0}^{t-s-1} a_{1}^{k} \omega_{i, t-k} a_{1}^{s+k-t} \sum_{m=0}^{\infty} a_{1}^{m} \omega_{i, t-k-m}\right)=0$.

It follows that $\lim _{a_{1} \uparrow 1}\left(a_{1}-1\right)^{2} E\left(\sum_{m=0}^{t-s-1} a_{1}^{m} \omega_{i, t-m}\right)\left(\sum_{m=0}^{\infty} a_{1}^{m} \omega_{i, s-m}\right)=0$.

Using previous results, we find that $\lim _{a_{1} \uparrow 1} E\left(s, t, a_{1}, 1\right)=0 \forall s, t$.

We will now show that $\lim _{a_{1} \uparrow 1} E\left(s, t, a_{1}, 2\right)=0 \forall s, t$.

First, $E\left(s, t, a_{1}, 2\right)=E\left[\left(\sum_{m=0}^{\infty} a_{1}^{m}\left(a_{1}-1\right) \omega_{i, s-1-m}\right)\left(\omega_{i, t}\right)\right]=$

$E\left[\left(\sum_{m=s-t-1}^{\infty} a_{1}^{m}\left(a_{1}-1\right) \omega_{i, s-1-m}\right)\left(\omega_{i, t}\right)\right]-E\left[\left(\sum_{m=s-t-1}^{-1} a_{1}^{m}\left(a_{1}-1\right) \omega_{i, s-1-m}\right)\left(\omega_{i, t}\right)\right]=$

$a_{1}^{s-t-1} E\left(t, a_{1}\right)-E\left[\left(\sum_{m=s-t-1}^{-1} a_{1}^{m}\left(a_{1}-1\right) \omega_{i, s-1-m}\right)\left(\omega_{i, t}\right)\right]=$

$a_{1}^{s-t-1} E\left(t, a_{1}\right)-\left(a_{1}-1\right) E\left[\left(\sum_{m=s-t-1}^{-1} a_{1}^{m} \omega_{i, s-1-m}\right)\left(\omega_{i, t}\right)\right]$.

The sum consists of a finite number of terms. Stationarity of $\left\{\omega_{i, t}\right\}$ implies that $\lim _{a_{1} \uparrow 1}\left(1-a_{1}\right) E\left[\sum_{m=s-t-1}^{-1} a_{1}^{m} \omega_{i, s-1-m} \omega_{i, t}\right]=0$. Above, it was shown that $\lim _{a_{1} \uparrow 1} E\left(t, a_{1}\right)=0$ $\forall t$. It follows that $\lim _{a_{1} \uparrow 1} E\left(s, t, a_{1}, 2\right)=0 \forall s, t$.

Likewise, we can show that $\lim _{a_{1} \uparrow 1} E\left(s, t, a_{1}, 3\right)=0 \forall s, t$.

Recall that $E\left(s, t, a_{1}, 3\right)=E\left[\left(\omega_{i, s}\right)\left(\sum_{m=0}^{\infty} a_{1}^{m}\left(a_{1}-1\right) \omega_{i, t-1-m}\right)\right]$.

If $s=t$, then $E\left(s, t, a_{1}, 3\right)=E\left(s, a_{1}\right)$. Next, assume that $s \leq t-1$.

In that case $E\left[\left(\omega_{i, s}\right)\left(\sum_{m=0}^{\infty} a_{1}^{m}\left(a_{1}-1\right) \omega_{i, t-1-m}\right)\right]=$

$a_{1}^{t-s-1} E\left(s, a_{1}\right)+E\left[\left(\sum_{m=0}^{t-s-2} a_{1}^{m}\left(a_{1}-1\right) \omega_{i, t-1-m}\right)\left(\omega_{i, s}\right)\right]=$

$a_{1}^{t-s-1} E\left(s, a_{1}\right)+\left(a_{1}-1\right) E\left[\left(\sum_{m=0}^{t-s-2} a_{1}^{m} \omega_{i, t-1-m}\right)\left(\omega_{i, s}\right)\right]$.

We can show that $\lim _{a_{1} \uparrow 1}\left(1-a_{1}\right) E\left[\left(\sum_{m=0}^{t-s-2} a_{1}^{m} \omega_{i, t-1-m}\right)\left(\omega_{i, s}\right)\right]=0$ using that the sum consists of a finite number of terms and $\left\{\omega_{i, t}\right\}$ is a stationary process. In addition, $\lim _{a_{1} \uparrow 1} E\left(s, a_{1}\right)=0 \forall s$. We conclude that $\lim _{a_{1} \uparrow 1} E\left(s, t, a_{1}, 3\right)=0 \forall s, t$. 
Using the results from above, we obtain $\lim _{a_{1} \uparrow 1} E\left(s, t, a_{1}\right)=E\left(\omega_{i, s} \omega_{i, t}\right) \forall s, t$ with $|t-s|<\infty$. When $a_{1}=1, \Delta y_{i, t}=\omega_{i, t} \forall t$, and therefore $E\left(\Delta y_{i, s} \Delta y_{i, t}\right)=E\left(\omega_{i, s} \omega_{i, t}\right) \forall s, t$. It follows that $\lim _{n \rightarrow \infty} R\left(\check{r}_{n}\right)=R(r)$ for every $r \in \Theta_{r}^{U R Z}$.

Let us now prove the lemma for $R=D V D^{\prime}$, where $V$ is an arbitrary $\operatorname{ARMA}(\mathrm{p}, \mathrm{q})$ covariance matrix.

VdL shows that an $T \times T \operatorname{ARMA}(\mathrm{p}, \mathrm{q})$ matrix $V_{T}$ can be factorized as $V_{T}=K_{T} W_{T+q}^{-1} K_{T}^{\prime}$, where $K_{T}$ is an $T \times(T+q)$ matrix which is linear in the MA parameters and $W_{T+q}^{-1}$ is an $(T+$ $q) \times(T+q) \operatorname{AR}(\mathrm{p})$ covariance matrix. Let $\varphi_{1}, \ldots \varphi_{q}$ denote the MA parameters and let $O_{T, k}$ denote a $T \times k$ matrix of zeros, then we can write $K_{T}=\left[O_{T, q} I_{T}\right]+\sum_{k=1}^{q} \varphi_{k}\left[O_{T, q-k} I_{T} O_{T, k}\right]$. Noting that the $(T-1) \times T$ first difference matrix $D_{T}=\left[\begin{array}{ll}I_{T-1} & 0\end{array}\right]-\left[\begin{array}{ll}0 & I_{T-1}\end{array}\right]$, it can easily be verified that $D_{T} K_{T}=K_{T-1} D_{T+q}$. We obtain that $D_{T} V_{T} D_{T}^{\prime}=K_{T-1} D_{T+q} W_{T+q}^{-1} D_{T+q}^{\prime} K_{T-1}^{\prime}$.

Above we have shown that $\lim _{n \rightarrow \infty} \tilde{R}\left(\check{r}_{n}\right)=\tilde{R}(r)$ where $\tilde{R}(r)=D_{T} W_{T}^{-1} D_{T}^{\prime}$, for every $r \in \Theta_{r}^{U R Z}$ and for arbitrary $p, T<\infty$. Since $K_{T-1}$ is linear in (the elements of) $r, K_{T-1}$ is continuous in $r$. Finally, we notice that the product of left-continuous functions is leftcontinuous itself.

When $a_{1}=1, E\left(D y_{i} y_{i}^{\prime} D^{\prime}\right)=E\left(\omega_{i} \omega_{i}^{\prime}\right)$ is a stationary $\operatorname{ARMA}(\mathrm{p}-1, \mathrm{q})$ covariance matrix. Since all stationary ARMA matrices are nonsingular (see VdL) and bounded, $\left\|E\left(\omega_{i} \omega_{i}^{\prime}\right)\right\|<\infty$ and $E\left(\omega_{i} \omega_{i}^{\prime}\right)$ is nonsingular or more precisely $\sup _{r \in \Theta_{r}^{U R}}\|R(r)\|<\infty$, and $R(r)$ is nonsingular $\forall r \in \Theta_{r}^{U R}$, where $\|$.$\| is the Euclidean matrix norm.$

We will now show that $l_{C}\left(y ; r, \sigma^{2} \mid \widetilde{\mu}_{i}\right)$ is differentiable with respect to $\theta$.

It follows from VdL that the covariance matrix of a stationary ARMA process, (a stationary ARMA matrix for short) is nonsingular and bounded, that its elements are ratios of polynomials in the AR and MA parameters, and that its elements are differentiable with respect to the AR and MA parameters $(r)$ in every $\check{r} \in \Theta_{r}^{S}$ (the stationary parameter space). It also follows from VdL that the determinant and the inverse of a stationary ARMA matrix are differentiable with respect to $r$ in every $\check{r} \in \Theta_{r}^{S}$. In addition, the inverse and the determinant of the stationary ARMA matrix are bounded because the original matrix is nonsingular and its elements are bounded. Since the stationary ARMA matrices and their inverses and determinants consist of ratios of polynomials in the AR and MA parameters, they are in fact infinitely many times differentiable with respect to $r$ in every $\check{r} \in \Theta_{r}^{S}$.

At this point it should be noted that although $D V D^{\prime}$ is a stationary $\operatorname{ARMA}(\mathrm{p}, \mathrm{q}+1)$ 
matrix when $\left\{y_{t}\right\}$ is an $\operatorname{ARMA}(\mathrm{p}, \mathrm{q})$ process and $r^{S}<1$ or a stationary $\operatorname{ARMA}(\mathrm{p}-1, \mathrm{q})$ matrix when $r^{S}=1$, this matrix correponds to the difference of an ARMA process and it is therefore parametrized in a different way than the "standard" $\operatorname{ARMA}(\mathrm{p}, \mathrm{q}+1)$ or $\operatorname{ARMA}(\mathrm{p}-1, \mathrm{q})$ matrices found in VdL. In the case that $r^{S}<1$, one of the MA parameters is already known, so in that case the $\operatorname{ARMA}(\mathrm{p}, \mathrm{q}+1)$ matrix $D V D^{\prime}$ depends on only q MA parameters. In the unit root case, the $\operatorname{ARMA}(\mathrm{p}-1, \mathrm{q})$ matrix $D V D^{\prime}$ contains $\mathrm{p} A R$ parameters that satisfy the constraint $r^{S}=1$.

Now, $\forall r \in \Theta_{r}$ (including every $r \in \Theta_{r}^{U R}$ ) $R(r)=D V D^{\prime}$ exists and is stationary and hence bounded and also nonsingular. Furthermore, $\forall r \in \Theta_{r} R(r)$ consists of ratios of polynomials in the AR and MA parameters just like $V$. Because $R(r)$ is bounded, we have $\forall r \in \Theta_{r}$ that the denominators of $R(r)$ do not equal zero. Since polynomials are continuous and since the function $1 / x$ is continuous in every $x \in \Re, x \neq 0$, it follows that $\forall \varepsilon$ and $\forall r \in \Theta_{r}^{U R}, \exists \delta$ such that $\|R(r)-R(\check{r})\|<\varepsilon \forall \check{r}$ that satisfy $\|r-\check{r}\|<\delta$. So $\forall r \in \Theta_{r}^{U R}$, $\exists \delta(r)$ such that $R(r)$ is bounded and positive definite on $\mathcal{N}^{\delta}(r) \equiv\{\check{r} \mid\|r-\check{r}\|<\delta(r)\}$. Let $\Theta_{r}^{\delta}=\left(\bigcup_{r \in \Theta_{r}^{U R}} \mathcal{N}^{\delta}(r)\right) \cup \Theta_{r}$. It follows that $R(r)$ is differentiable with respect to $r$, i.e. with respect to the AR and MA parameters, in every $r \in \Theta_{r}^{\delta}$. Since the elements of the inverse of a matrix consist of ratios of minors and the determinant, the elements of $R(r)^{-1}$ also consist of ratios of polynomials in the AR and MA parameters. Because $R(r)$ is nonsingular and differentiable with respect to $r$ in every $r \in \Theta_{r}^{\delta}, R(r)^{-1}$ and $|R(r)|$ are also differentiable with respect to $r$ in every $r \in \Theta_{r}^{\delta}$. Moreover, $R(r)^{-1}$ and $|R(r)|$ are bounded on any compact subset of $\Theta^{\delta}$. Because the matrices and their inverses and the determinant consist of ratios of polynomials in the AR and MA parameters, they are in fact infinitely many times differentiable with respect to $r$ in every $r \in \Theta_{r}^{\delta}$.

We have just proven the following result

Lemma 12 The conditional likelihood function

$$
\begin{array}{r}
l_{C}\left(y ; r, \sigma^{2} \mid \widetilde{\mu}_{i}\right)=-\frac{N(T-1)}{2} \log (2 \pi)-\frac{N(T-1)}{2} \log \sigma^{2} \\
-\frac{N}{2} \log \left|D V D^{\prime}\right|-\frac{1}{2 \sigma^{2}} \sum_{i=1}^{N}\left(\left(y_{i}-\mu_{i}\right)^{\prime} D^{\prime}\left(D V D^{\prime}\right)^{-1} D\left(y_{i}-\mu_{i}\right)\right)
\end{array}
$$

is infinitely many times differentiable with respect to $\theta$ in every $\check{\theta} \in \Theta^{\delta}$. 
The function $L_{C}\left(y_{i} ; r, \sigma^{2} \mid \widetilde{\mu}_{i}\right)$ is a normal probability density function (p.d.f.) $\forall \theta \in \Theta^{\delta}$, because $R(r)$ is positive definite symmetric $\forall r \in \Theta_{r}^{\delta}$. However, $\forall \theta \in \Theta^{\delta}$ with $r^{S}>1$, this density is not the p.d.f. of $D y_{i}$.

Below we will give a consistency proof for CMLE's for general inclusive $\operatorname{ARMAFE}(\mathrm{p}, \mathrm{q})$ models. In the following we first present an alternative proof of the consistency of the CMLE for the inclusive ARFE(1) model. The reason for including this alternative proof is that the ARFE(1) model is simple enough to allow us to address some technical issues using explicit expressions.

\section{A.1 The conditional likelihood function for the ARFE(1) model is well-defined when $r=1$ :}

In section 2 we have shown that the conditional log-likelihood function (6) is equal to the log-likelihood function for first differences (8). When $V$ is pre- and postmultiplied by $D$ and $D^{\prime}$ respectively, the factor $1-r$ from the denominator of $V$ cancels out. Moreover, the $\operatorname{ARMA}(1,1)$ covariance matrix $R(r)=D V D^{\prime}$ is (left-)continuous in $r=1(R(1)=I$, the $\operatorname{MA}(0)$ covariance matrix), and also differentiable in $r=1$. The matrix $\left(D V D^{\prime}\right)^{-1}$ and $\left|D V D^{\prime}\right|$ are also differentiable in $r=1$.

\section{A.2 Consistency of the CMLE for $r$ and $\sigma^{2}$ in the model given by (2) and (3) with $\left(r \sigma^{2}\right)^{\prime}$ belonging to a compact subset of $\Theta=(-1,1] \times(0, \infty):$}

To prove consistency of the CMLE we will verify the conditions of theorem 2.5. in Newey and McFadden (1994).

We can easily find a compact subset of the parameter space $\Theta$ for $\theta=\left(r^{\prime} \sigma^{2}\right)^{\prime}$, i.e. take $\bar{\Theta}=\left[-1+\delta_{1}, 1\right] \times\left[\delta_{2}, \frac{1}{\delta_{2}}\right]$ with $\delta_{1}, \delta_{2}$ very small positive numbers.

$l_{C}\left(y_{i} ; r, \sigma^{2} \mid \widetilde{\mu}_{i}\right)$ is continuous on $\bar{\Theta}$.

Upperbars denote true values of parameters.

To show that the parameters $\bar{r}$ and $\bar{\sigma}^{2}$ are uniquely identified, i.e. $\operatorname{Prob}\left(\left\{y_{i}: l_{C}\left(y_{i} ; \theta \mid \widetilde{\mu}_{i}\right) \neq\right.\right.$ $\left.\left.l_{C}\left(y_{i} ; \bar{\theta} \mid \widetilde{\mu}_{i}\right)\right\}\right)=1$ if and only if (iff for short) $\theta \neq \bar{\theta}$, we consider the exponential part of 
$l_{C}\left(y_{i} ; \theta \mid \widetilde{\mu}_{i}\right)$, more in particular

$$
M(r) \equiv D^{\prime}\left(D V D^{\prime}\right)^{-1} D=V(r)^{-1}-\frac{V(r)^{-1} \iota \iota^{\prime} V(r)^{-1}}{\iota^{\prime} V(r)^{-1} \iota}
$$

where the latter equality can be found in Lancaster and Lindenhovius (1996). It still holds for $r=1$. Now $V(r)^{-1}$ has a very simple structure:

$$
\begin{aligned}
V_{i i}^{-1} & =1 \text { for } i=1, i=T \\
V_{i i}^{-1} & =1+r^{2} \text { for } i=2, \ldots, T-1 \\
V_{i j}^{-1} & =-r \text { for } j=i+1, j=i-1 \\
V_{i j}^{-1} & =0 \text { elsewhere }
\end{aligned}
$$

It turns out that the matrix $M$ contains 2 , (when $T=3), 4($ when $T=4), 6$ (when $T=5$ ) or 7 (when $T>5$ ) different kind of elements, which are all ratios of polynomial functions of $r$. The denominator of these ratios is always equal to $T(1-r)+2 r>0$ for $-1<r \leq 1$, while the numerators are linear, quadratic and cubic functions of $r$. Therefore it can easily be shown that $M(r) \neq M(\bar{r})$ iff $r \neq \bar{r}$ when $T>2$. Given identification of $r$, identification of $\sigma^{2}$ is trivial. When $T=2$ only $\frac{1+r}{\sigma^{2}}$ can be identified.

Although the parameters $\bar{r}$ and $\bar{\sigma}^{2}$ are uniquely identified, it should be clear from the previous paragraph that $l_{C}\left(y_{i} ; \theta \mid \widetilde{\mu}_{i}\right)$ and $E\left(l_{C}\left(y_{i} ; \theta \mid \widetilde{\mu}_{i}\right)\right)$ admit multiple local maxima on $\mathcal{R}$ $\times \mathcal{R}_{+}$(unless $T$ is very small).

Finally, we can rewrite the conditional log-likelihood as

$$
\begin{aligned}
& l_{C}\left(y_{i} ; r, \sigma^{2} \mid \widetilde{\mu}_{i}\right)=-\frac{(T-1)}{2} \log (2 \pi)-\frac{(T-1)}{2} \log \sigma^{2} \\
& -\frac{1}{2} \log \left|D V D^{\prime}\right|-\frac{1}{2 \sigma^{2}} \operatorname{tr}\left(\left(D V D^{\prime}\right)^{-1} D y_{i} y_{i}^{\prime} D^{\prime}\right)
\end{aligned}
$$

To show that $E\left(\sup _{\left(r, \sigma^{2}\right) \in \bar{\Theta}} l_{C}\left(y_{i} ; r, \sigma^{2} \mid \widetilde{\mu}_{i}\right)\right)<\infty$, we first notice that

$$
\left|D V(r) D^{\prime}\right|=\frac{(T(1-r)+2 r)}{1+r}>0
$$

and bounded on the interval $(-1,1]$. $\left(r=-1\right.$ has been ruled out). It follows that $\left(D V(r) D^{\prime}\right)^{-1}$ exists and is bounded for $-1<r \leq 1$. Furthermore $E\left(D y_{i} y_{i}^{\prime} D^{\prime}\right)=\bar{\sigma}^{2} D V(\bar{r}) D^{\prime}$ is bounded by stationarity of $D y_{i}$. 


\section{A.3 Consistency of the CMLE for the inclusive $\operatorname{ARMAFE}(p, q)$ model}

To prove consistency of the CMLE we will verify the conditions of theorem 2.5. in Newey and McFadden (1994).

We assume that $\bar{\theta} \in \bar{\Theta} \subset \Theta$, where $\bar{\Theta}$ can be any compact subset of $\Theta$. However we will assume that $\bar{\Theta}$ is such that $\Theta_{r}^{U R Z} \subset \bar{\Theta}_{r}$.

We have already shown that $l_{C}\left(y_{i} ; r, \sigma^{2} \mid \widetilde{\mu}_{i}\right)$ is differentiable with respect to $r$ in every $\theta \in \Theta^{\delta}$. It is then easily shown that $l_{C}\left(y_{i} ; r, \sigma^{2} \mid \widetilde{\mu}_{i}\right)$ is differentiable with respect to $\theta$ in every $\theta \in \Theta^{\delta}$. It follows that $l_{C}\left(y_{i} ; r, \sigma^{2} \mid \widetilde{\mu}_{i}\right)$ is continuous in every $\theta \in \bar{\Theta}$.

The last two steps of the proof are as follows.

For identification it suffices to show that $\sigma^{2} D V(r) D^{\prime} \neq \bar{\sigma}^{2} D V(\bar{r}) D^{\prime} \forall \theta \in \bar{\Theta}$ with $\theta \neq \bar{\theta}$, because $\left(\sigma^{2} D V(r) D^{\prime} \neq \bar{\sigma}^{2} D V(\bar{r}) D^{\prime}\right.$ if and only if $\left.\theta \neq \bar{\theta}\right) \Leftrightarrow\left(\left(\sigma^{2} D V(r) D^{\prime}\right)^{-1} \neq\right.$ $\left(\bar{\sigma}^{2} D V(\bar{r}) D^{\prime}\right)^{-1}$ iff $\left.\theta \neq \bar{\theta}\right)$. The latter follows trivially from the fact that $\sigma^{2} D V(r) D^{\prime} \times$ $\left(\sigma^{2} D V(r) D^{\prime}\right)^{-1}=I \forall \theta \in \bar{\Theta}$. Now, the $T-1$ elements of the first column of the (stationary) covariance matrix $\sigma^{2} D V D^{\prime}$ are all different functions of the $p+q+1$ parameters. It follows that if $p+q+1 \leq T-1,\left(\sigma^{2} D V(r) D^{\prime} \neq \bar{\sigma}^{2} D V(\bar{r}) D^{\prime}\right) \Leftrightarrow \theta \neq \bar{\theta}$.

To show that $E\left(\sup _{\left(r, \sigma^{2}\right) \in \bar{\Theta}} l_{C}\left(y_{i} ; r, \sigma^{2} \mid \widetilde{\mu}_{i}\right)\right)<\infty$, we recall that $\left|\sigma^{2} D V(r) D^{\prime}\right|>0 \forall \theta \in \Theta^{\delta}$ and that $\left(D V(r) D^{\prime}\right)^{-1}$ and $\left|D V(r) D^{\prime}\right|$ are differentiable and hence continuous in every $r \in \Theta_{r}^{\delta}$. It follows that $\sigma^{-2}\left(D V(r) D^{\prime}\right)^{-1}$ and $\left|\sigma^{2} D V(r) D^{\prime}\right|$ are bounded on the compact set $\bar{\Theta}$. Futhermore, $E\left(D y_{i} y_{i}^{\prime} D^{\prime}\right)$ is bounded by stationarity of $D y_{i}$. We conclude that $E\left(\sup _{\left(r, \sigma^{2}\right) \in \bar{\Theta}} l_{C}\left(y_{i} ; r, \sigma^{2} \mid \widetilde{\mu}_{i}\right)\right)<\infty$.

\section{A.4 Asymptotic normality of the CMLE for the inclusive AR- $\operatorname{MAFE}(p, q)$ model}

Asymptotic normality of the CMLE for the inclusive $\operatorname{ARMAFE}(p, q)$ model can be established by verifying the conditions of theorem 3.3. in Newey and McFadden (1994), henceforth NMcF. The proof proceeds as follows:

Above we have seen that $\forall r \in \Theta_{r}^{U R}, \exists \delta(r)$ such that $R(r)$ is bounded and positive definite on $\mathcal{N}^{\delta}(r)=\{\check{r} \mid\|r-\check{r}\|<\delta(r)\}$. Also recall that $\Theta_{r}^{\delta}=\left(\bigcup_{r \in \Theta_{r}^{U R}} \mathcal{N}^{\delta}(r)\right) \cup \Theta_{r}$. 
Let us now define the compact set $\overline{\mathcal{N}}^{\frac{1}{2} \delta}(\bar{r}) \equiv\left\{\check{r} \mid\|\bar{r}-\check{r}\| \leq \frac{1}{2} \delta(\bar{r})\right\}$, where $\bar{r}$ is the true value of $r$. Next, let $\bar{\Theta}_{\bar{r}}^{\frac{1}{2} \delta}=\overline{\mathcal{N}}^{\frac{1}{2} \delta}(\bar{r}) \cup \bar{\Theta}_{r}$, where $\bar{\Theta}_{r}$ is a compact set with $\Theta_{r}^{U R Z} \subset \bar{\Theta}_{r} \subset \Theta_{r}$. Note that $\bar{\Theta}_{\bar{r}}^{\frac{1}{2} \delta} \subset \Theta_{r}^{\delta}$.

The true value $\bar{\theta} \in \bar{\Theta}_{r} \times\left[\delta_{2}, \frac{1}{\delta_{2}}\right]$ where $\delta_{2}>0$ and very small. It follows that $\bar{\theta} \in$ interior $\left(\bar{\Theta}_{\frac{2}{r}}^{\frac{1}{2} \delta} \times\left[\frac{1}{2} \delta_{2}, \frac{2}{\delta_{2}}\right]\right)$. We note that the conditions of theorem 2.5 in NMcF are also satisfied for the larger compact set $\bar{\Theta}_{\bar{r}}^{\frac{1}{2} \delta} \times\left[\frac{1}{2} \delta_{2}, \frac{2}{\delta_{2}}\right]$ (of course, we still assume that the true value $\bar{\theta} \in \bar{\Theta}_{r} \times\left[\delta_{2}, \frac{1}{\delta_{2}}\right]$, e.g. when evaluating expectations). It can also be verified that lemma 2.2 and lemma 2.4 of NMcF are still applicable when we use the larger compact set $\bar{\Theta}_{\bar{r}}^{\frac{1}{2} \delta} \times\left[\frac{1}{2} \delta_{2}, \frac{2}{\delta_{2}}\right]$.

Above we have shown that $L_{C}\left(y_{i} ; r, \sigma^{2} \mid \widetilde{\mu}_{i}\right)$ is infinitely many times differentiable with respect to AR and MA parameters in every $\theta \in \Theta^{\delta}$. Furthermore we have observed that $L_{C}\left(y_{i} ; r, \sigma^{2} \mid \widetilde{\mu}_{i}\right)$ is a proper normal p.d.f. $\forall \theta \in \Theta^{\delta}$ and therefore $L_{C}\left(y_{i} ; r, \sigma^{2} \mid \widetilde{\mu}_{i}\right)>0$ on $\Theta^{\delta}$.

The Hessian of $l_{C}\left(y_{i} ; r, \sigma^{2} \mid \widetilde{\mu}_{i}\right)$ is given by (cf Magnus (1978)) $H\left(\theta, y_{i}\right)=\frac{1}{2} \sum_{1 \leq s, t<T}\left(\Omega_{s, t}-\Delta y_{i, s+1} \Delta y_{i, t+1}\right) \frac{\partial^{2}\left[\Omega^{-1}\right]_{s, t}}{\partial \theta \partial \theta^{\prime}}-\frac{1}{2}\left(\left[\left.\frac{d v e c \Omega}{d \theta^{\prime}}\right|_{\bar{\theta}}\right]^{\prime}\left(\bar{\Omega}^{-1} \otimes \bar{\Omega}^{-1}\right)\left[\left.\frac{d v e c \Omega}{d \theta^{\prime}}\right|_{\bar{\theta}}\right]\right)$.

Above we have shown that $R(r)$ is nonsingular and that $R(r), R(r)^{-1}$, and $|R(r)|$ are ratios of polynomials in the elements of $r$ and infinitely many times differentiable on $\Theta^{\delta}$. It follows that $R(r), R(r)^{-1}$, and $|R(r)|$ and their derivatives with respect to $r$ are bounded on any compact subset of $\Theta^{\delta}$. We also noticed that $L_{C}\left(y_{i} ; r, \sigma^{2} \mid \widetilde{\mu}_{i}\right)$ is a proper p.d.f. $\forall \theta \in \Theta^{\delta}$. These results together with stationarity of $D y_{i}$, which gives that $E\left(D y_{i} y_{i}^{\prime} D^{\prime}\right)$ is bounded, and the fact that the standard normal density $\phi(v)$ and its first and second derivatives are uniformly bounded imply that conditions (iii) and (v) of theorem $3.3 \mathrm{in} \mathrm{NMcF}$ are satisfied ( since we can choose the neighbourhood of $\bar{\theta}, \mathcal{N}(\bar{\theta})$, such that $\mathcal{N}(\bar{\theta}) \subset \bar{\Theta}_{\bar{r}}^{\frac{1}{2} \delta} \times\left[\frac{1}{2} \delta_{2}, \frac{2}{\delta_{2}}\right]$ ).

The second Bartlett identity holds because condition (iii) of theorem 3.3 in NMcF allows us to interchange the order of differentiation and integration. So the information matrix equals minus the expected value of the Hessian of $l_{C}\left(y_{i} ; r, \sigma^{2} \mid \widetilde{\mu}_{i}\right)$, i.e. $\frac{1}{2}\left(\left[\left.\frac{d v e c \Omega}{d \theta^{\prime}}\right|_{\bar{\theta}}\right]^{\prime}\left(\bar{\Omega}^{-1} \otimes\right.\right.$ $\left.\left.\bar{\Omega}^{-1}\right)\left[\frac{d v e c \Omega}{d \theta^{\prime}} \mid \bar{\theta}\right]\right)$. Using the results concerning $R(r)^{-1}$ from above we can easily establish existence of the information matrix for any $\bar{\theta} \in \Theta$. Finally, the information matrix is nonsingular if $\frac{d v e c h \Omega}{d \theta^{\prime}}$ has full rank. Because $\Omega$ has the structure of a covariance matrix of a stationary process, it is a Toeplitz matrix and therefore we can restrict attention to the derivatives of the elements of the first column of $\Omega$. The fact that the elements of $\theta$ can be identified from 
this column when $T-1 \geq p+q+1$ implies that $\frac{d v e c h \Omega}{d \theta^{\prime}}$ has full rank when $T-1 \geq p+q+1$.

\section{A.5 Asymptotic distribution of the CMLE for the inclusive au- toregressive model with fixed effects}

In this appendix we will derive the asymptotic distribution of the CML estimator for the inclusive ARFE(1) model.

First, we will introduce some notation. Let $u_{i} \equiv y_{i}-\mu_{i} \iota, f(r) \equiv \iota^{\prime} V^{-1} \iota$, and $b(r)^{\prime} \equiv \frac{\iota^{\prime} V^{-1}}{\iota^{\prime} V^{-1} \iota}$ ( $\equiv b^{\prime}$ for short). The log-likelihood function of $\widetilde{\mu}_{i}=b^{\prime} y_{i}$ is

$$
\begin{aligned}
l\left(\widetilde{\mu}_{i}\right) & =-\frac{1}{2} \log 2 \pi-\frac{1}{2} \log \sigma^{2}+\frac{1}{2} \log f(r)-\frac{f(r)}{2 \sigma^{2}}\left(\widetilde{\mu}_{i}-\mu_{i}\right)^{2} \\
& =-\frac{1}{2} \log 2 \pi-\frac{1}{2} \log \sigma^{2}+\frac{1}{2} \log f(r)-\frac{f(r)}{2 \sigma^{2}}\left(b(r)^{\prime}\left(y_{i}-\mu_{i} \iota\right)\right)^{2}
\end{aligned}
$$

Then in case of the ARFE(1) model the elements of the Expected Hessian of this (Marginal) log-likelihood function, EHM, are obtained as follows ${ }^{34}$

$$
\begin{aligned}
& \frac{d l\left(\widetilde{\mu}_{i}\right)}{d r}=\frac{1}{2} \frac{f^{\prime}(r)}{f(r)}-\frac{f^{\prime}(r)}{2 \sigma^{2}}\left(b(r)^{\prime}\left(y_{i}-\mu_{i} \iota\right)\right)^{2}-\frac{f(r)}{\sigma^{2}} b(r)^{\prime}\left(y_{i}-\mu_{i} \iota\right) \frac{d b(r)^{\prime}}{d r}\left(y_{i}-\mu_{i} \iota\right) \\
& \frac{d^{2} l\left(\widetilde{\mu}_{i}\right)}{d r^{2}}=\frac{1}{2}\left[\frac{f^{\prime \prime}(r)}{f(r)}-\left(\frac{f^{\prime}(r)}{f(r)}\right)^{2}\right]-\frac{f^{\prime \prime}(r)}{2 \sigma^{2}}\left(\widetilde{\mu}_{i}-\mu_{i}\right)^{2}-2 \frac{f^{\prime}(r)}{\sigma^{2}} b(r)^{\prime}\left(y_{i}-\mu_{i} \iota\right) \frac{d b(r)^{\prime}}{d r}\left(y_{i}-\mu_{i} \iota\right) \\
& -\frac{f(r)}{\sigma^{2}}\left(\frac{d b(r)^{\prime}}{d r}\left(y_{i}-\mu_{i} \iota\right)\right)^{2}-\frac{f(r)}{\sigma^{2}} b(r)^{\prime}\left(y_{i}-\mu_{i} \iota\right) \frac{d^{2} b(r)^{\prime}}{d r^{2}}\left(y_{i}-\mu_{i} \iota\right) .
\end{aligned}
$$

Now, we have

$$
\begin{aligned}
& E\left(\frac{f^{\prime \prime}(r)}{2 \sigma^{2}}\left(\widetilde{\mu}_{i}-\mu_{i}\right)^{2}\right)=\frac{1}{2} \frac{f^{\prime \prime}(r)}{f(r)}, \\
& E\left(b(r)^{\prime}\left(y_{i}-\mu_{i} \iota\right) \frac{d b(r)^{\prime}}{d r}\left(y_{i}-\mu_{i} \iota\right)\right)=E\left(b(r)^{\prime} u_{i} u_{i}^{\prime} \frac{d b(r)}{d r}\right)=\sigma^{2} b(r)^{\prime} V \frac{d b(r)}{d r}= \\
& \frac{\sigma^{2}}{c} \iota^{\prime} \frac{d b(r)}{d r}=0 .
\end{aligned}
$$

Likewise, $E\left(b(r)^{\prime}\left(y_{i}-\mu_{i} \iota\right) \frac{d^{2} b(r)^{\prime}}{d r^{2}}\left(y_{i}-\mu_{i} \iota\right)\right)=0$.

$$
\begin{aligned}
& E\left(\frac{f(r)}{\sigma^{2}}\left(\frac{d b(r)^{\prime}}{d r}\left(y_{i}-\mu_{i} \iota\right)\right)^{2}\right)=E\left(\frac{f(r)}{\sigma^{2}}\left(\frac{d b(r)^{\prime}}{d r}\left(u_{i} u_{i}^{\prime}\right) \frac{d b(r)}{d r}\right)\right)=E\left(f(r) \frac{d b(r)^{\prime}}{d r} V \frac{d b(r)}{d r}\right) \\
& =\frac{1}{f(r)} \frac{d\left(\iota^{\prime} V^{-1}\right)}{d r} V \frac{d\left(V^{-1} \iota\right)}{d r}-2 \frac{1}{f(r)} \frac{d\left(\iota^{\prime} V^{-1}\right)}{d r} V b(r) f^{\prime}(r)+\frac{1}{f(r)} b(r)^{\prime} V b(r)\left(f^{\prime}(r)\right)^{2}= \\
& \frac{1}{f(r)} \frac{d\left(\iota^{\prime} V^{-1}\right)}{d r} V \frac{d\left(V^{-1} \iota\right)}{d r}-2\left(\frac{1}{f(r)}\right)^{2} \frac{d\left(\iota^{\prime} V^{-1}\right)}{d r} \iota f^{\prime}(r)+\left(\frac{f^{\prime}(r)}{f(r)}\right)^{2}=
\end{aligned}
$$

\footnotetext{
${ }^{34}$ The prime' denotes a derivative in the case of a scalar function and transpose in case of a vector.
} 


$$
\frac{1}{f(r)} \frac{d\left(\iota^{\prime} V^{-1}\right)}{d r} V \frac{d\left(V^{-1} \iota\right)}{d r}-2\left(\frac{f^{\prime}(r)}{f(r)}\right)^{2}+\left(\frac{f^{\prime}(r)}{f(r)}\right)^{2}=\frac{1}{f(r)} \frac{d\left(\iota^{\prime} V^{-1}\right)}{d r} V \frac{d\left(V^{-1} \iota\right)}{d r}-\left(\frac{f^{\prime}(r)}{f(r)}\right)^{2} .
$$

Therefore, we obtain $E\left(\frac{d^{2} l\left(\tilde{\mu}_{i}\right)}{d r^{2}}\right)=-\frac{1}{f(r)} \frac{d\left(\iota^{\prime} V^{-1}\right)}{d r} V \frac{d\left(V^{-1} \iota\right)}{d r}+\frac{1}{2}\left(\frac{f^{\prime}(r)}{f(r)}\right)^{2}$.

In the $\operatorname{AR}(1)$ case $f(r)=\iota^{\prime} V^{-1} \iota=(1-r)((T-2)(1-r)+2)$ and

$\frac{d\left(\iota^{\prime} V^{-1}\right)}{d r} V \frac{d\left(V^{-1} \iota\right)}{d r}=\iota^{\prime} \frac{d V^{-1}}{d r} V \frac{d V^{-1}}{d r} \iota=\frac{2}{1-r^{2}}\left(r^{T-1}-2(T-2) r^{2}+2 T-3\right)$. The latter result is not obvious and has been obtained by using Mathematica and by exploiting the fact that $\frac{d V^{-1}}{d r} V \frac{d V^{-1}}{d r}$ has a particular structure for arbitrary values of $T$. The other elements of EHM are

$$
\begin{aligned}
& E\left(\frac{d^{2} l\left(\widetilde{\mu}_{i}\right)}{d r d \sigma^{2}}\right)=\frac{f^{\prime}(r)}{2 \sigma^{2} f(r)} E\left(\frac{d^{2} l\left(\widetilde{\mu}_{i}\right)}{d\left(\sigma^{2}\right)^{2}}\right)=-\frac{1}{2 \sigma^{4}} . \\
& E\left(\frac{d^{2} l\left(\widetilde{\mu}_{i}\right)}{d r d \mu_{i}}\right)=0 E\left(\frac{d^{2} l\left(\widetilde{\mu}_{i}\right)}{d \sigma^{2} d \mu_{i}}\right)=0 \text { and } E\left(\frac{d^{2} l\left(\widetilde{\mu}_{i}\right)}{d \mu_{i}^{2}}\right)=-\frac{f(r)}{\sigma^{2}} .
\end{aligned}
$$

We will now derive the expected Hessian corresponding to the unconditional likelihood function, EHU. The unconditional log-likelihood of the $y_{i}^{\prime} s$ is given by

$$
l\left(y_{i}\right)=-\frac{T}{2} \log (2 \pi)-\frac{T}{2} \log \sigma^{2}-\frac{1}{2} \log |V|-\frac{1}{2 \sigma^{2}} u_{i}^{\prime} V^{-1} u_{i}
$$

In the $\operatorname{AR}(1)$ case $|V|=\left(1-r^{2}\right)^{-1}$ and $V^{-1}$ has a very simple structure

$$
V_{T}^{-1}(r)=\left(\begin{array}{ccccccc}
1 & -r & 0 & . & . & . & 0 \\
-r & 1-r^{2} & -r & \cdot & . & . & 0 \\
0 & -r & 1-r^{2} & . & & & . \\
\cdot & . & . & . & . & & . \\
. & . & & . & . & . & 0 \\
. & . & & & . & 1-r^{2} & -r \\
0 & 0 & . & . & 0 & -r & 1
\end{array}\right) \text { with }|r|<1
$$

Therefore we obtain $E\left(\frac{d\left(u_{i}^{\prime} V^{-1} u_{i}\right)}{d r}\right)=-\sigma^{2} \frac{2 r}{1-r^{2}}$ and $E\left(\frac{d^{2}\left(u_{i}^{\prime} V^{-1} u_{i}\right)}{d r^{2}}\right)=\sigma^{2} \frac{2(T-2)}{1-r^{2}}$.

We also have $\frac{d|V|}{d r}=2 r(|V|)^{2}$ and $\frac{d^{2}|V|}{d r^{2}}=2(|V|)^{2}+8 r^{2}(|V|)^{3}$.

It follows that $E\left(\frac{d^{2} l\left(y_{i}\right)}{d r^{2}}\right)=-\left[\frac{(T-2)\left(1-r^{2}\right)+1+r^{2}}{\left(1-r^{2}\right)^{2}}\right]$ and $E\left(\frac{d^{2} l\left(y_{i}\right)}{d r d \sigma^{2}}\right)=-\frac{1}{\sigma^{2}} \frac{r}{1-r^{2}}$.

The other elements of EHU are:

$$
E\left(\frac{d^{2} l\left(y_{i}\right)}{d\left(\sigma^{2}\right)^{2}}\right)=-\frac{T}{2 \sigma^{4}} ; E\left(\frac{d^{2} l\left(y_{i}\right)}{d r d \mu_{i}}\right)=0 ; E\left(\frac{d^{2} l\left(y_{i}\right)}{d \sigma^{2} d \mu_{i}}\right)=0 ; \text { and } E\left(\frac{d^{2} l\left(y_{i}\right)}{d \mu_{i}^{2}}\right)=-\frac{f(r)}{\sigma^{2}}
$$

The last three elements of EHU are the same as those of EHM because $\widetilde{\mu}_{i}$ is a sufficient statistic.

The large-N asymptotic distribution of $\left\{\widehat{r}_{C M L}, \widehat{\sigma}_{C M L}^{2}\right\}$ is given by 


$$
\sqrt{N T}\left(\begin{array}{c}
\widehat{r}_{C M L}-r \\
\widehat{\sigma}_{C M L}^{2}-\sigma^{2}
\end{array}\right) \rightarrow N\left(0, T\left(E H M_{r, \sigma^{2}}-E H U_{r, \sigma^{2}}\right)^{-1}\right)
$$

An explicit expression for $\left(E H M_{r, \sigma^{2}}-E H U_{r, \sigma^{2}}\right)$ for the ARFE(1) model is given in the main text as formula (10) together with (11).

Below we have plotted graphs of the asymptotic variance of $\widehat{r}_{C M L}$ (normalized by $T$ ) (the CMLE for $r$ in the inclusive AR(1) model) for various values of $T$.

\section{Figure 1: the asymptotic variance of the CMLE for $r$}

$$
T=3 \quad f(r)=\frac{3}{4}(-3+r)^{2}(1+r)^{2}
$$

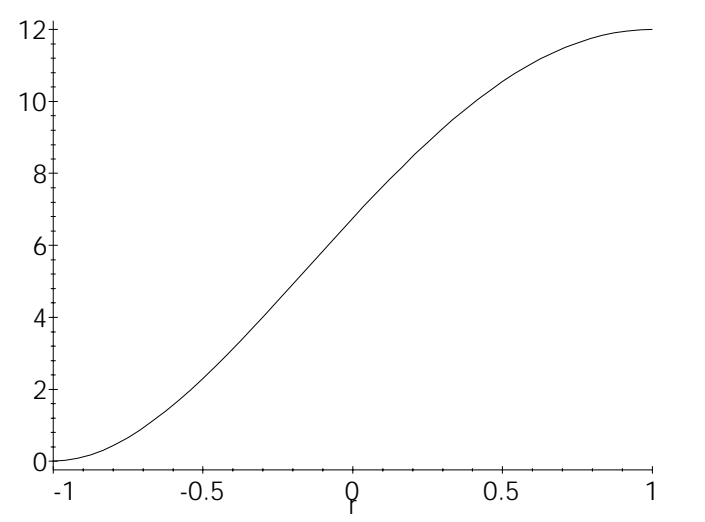

$$
T=8 \quad x(r)=8 \frac{(-4+3 r)^{2}(1+r)^{2}}{3 r^{7}+5 r^{6}+3 r^{5}+r^{4}-r^{3}-66 r^{2}-5 r+81}
$$

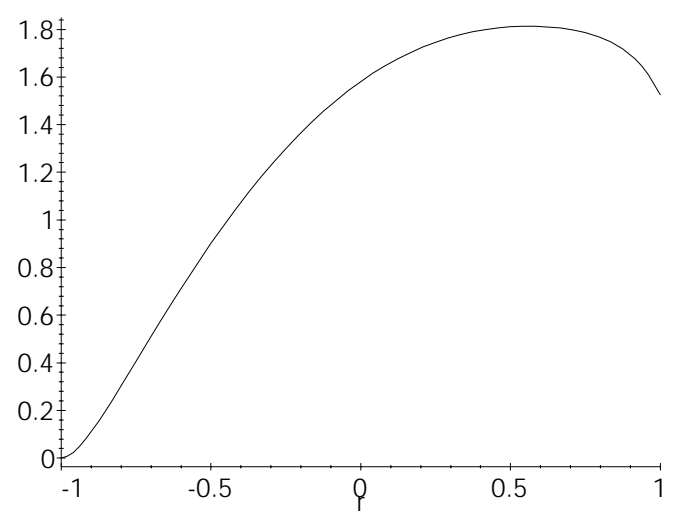




$$
\begin{gathered}
T=15 \\
y(r)=\frac{15}{2} \frac{(-15+13 r)^{2}(1+r)^{2}}{13 r^{14}+24 r^{13}+20 r^{12}+16 r^{11}+12 r^{10}+8 r^{9}+4 r^{8}-4 r^{6}-8 r^{5}-12 r^{4}-16 r^{3}-1203 r^{2}-24 r+1352}
\end{gathered}
$$

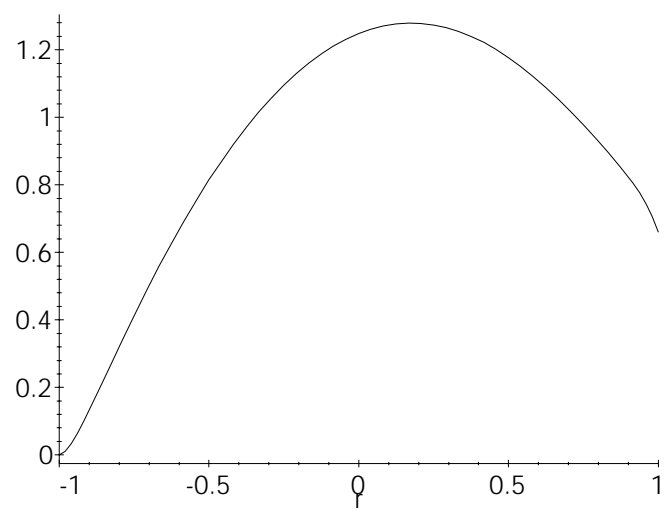

$$
T=\infty \quad k(r)=1-r^{2}
$$

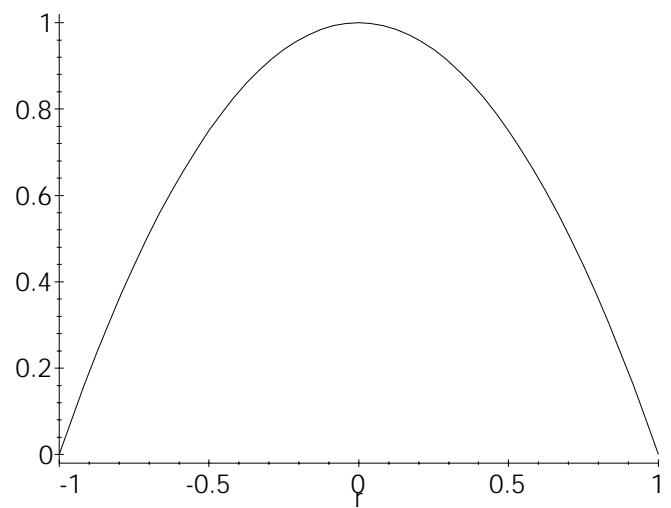




\section{B Proof of Results in SeCtion 3}

\section{B.1 Proof of Theorem 4}

The first part of the proof follows the reasoning of Cramér (1946) (see also Neyman and Scott, 1948). By using standard arguments it can be shown that the objective function $\frac{1}{N} \log p\left(r, \sigma^{2}\right)=\frac{1}{N} \sum_{i=1}^{N} \log p_{i}\left(r, \sigma^{2}\right)$ converges uniformly in probability to

$$
\begin{aligned}
& E \log p\left(r, \sigma^{2} ; \bar{r}, \bar{\sigma}^{2}\right)= \\
& \beta(r)-\frac{T-1}{2} \ln \sigma^{2}-\frac{1}{2 \sigma^{2}}\left(\lim _{N \rightarrow \infty} \frac{1}{N} \sum_{i=1}^{N} E\left(y_{i}-r y_{i,-1}\right)^{\prime} Q\left(y_{i}-r y_{i,-1}\right)\right)= \\
& \beta(r)-\frac{T-1}{2} \ln \sigma^{2}-\frac{\bar{\sigma}^{2}}{2 \sigma^{2}} \operatorname{tr}(Q) \\
& -\frac{1}{2 \sigma^{2}}\left((r-\bar{r})^{2}\left(\lim _{N \rightarrow \infty} \frac{1}{N} \sum_{i=1}^{N} E\left(y_{i,-1}^{\prime} Q y_{i,-1}\right)\right)-2(r-\bar{r}) E\left(\varepsilon_{i}^{\prime} Q y_{i,-1}\right)\right)
\end{aligned}
$$

where $p\left(r, \sigma^{2}\right)$ is given in (23), $\bar{r}$ and $\bar{\sigma}^{2}$ are the true values of $r$ and $\sigma^{2}$, and $\left(r \sigma^{2}\right) \in(-1,1) \times(0, \infty)$, under the additional assumptions that

$$
\lim _{N \rightarrow \infty} \frac{1}{N} \sum_{i=1}^{N} y_{i, 0}^{2}<\infty \quad \text { and } \quad \lim _{N \rightarrow \infty} \frac{1}{N} \sum_{i=1}^{N} \overline{\check{\mu}}_{i}^{2}<\infty .
$$

Let us define the modified likelihood equations

$$
\begin{aligned}
\Psi_{r, i}\left(r, \sigma^{2}\right) & \equiv \beta^{\prime}(r)+\frac{1}{\sigma^{2}}\left(y_{i}-r y_{i,-1}\right)^{\prime} Q y_{i,-1} \\
\Psi_{\sigma^{2}, i}\left(r, \sigma^{2}\right) & \equiv-\frac{T-1}{2 \sigma^{2}}+\frac{1}{2 \sigma^{4}}\left(y_{i}-r y_{i,-1}\right)^{\prime} Q\left(y_{i}-r y_{i,-1}\right)
\end{aligned}
$$

then

$$
\begin{aligned}
\Psi_{r, i}\left(\bar{r}, \bar{\sigma}^{2}\right) & =\beta^{\prime}(\bar{r})+\frac{\varepsilon_{i}^{\prime} Q y_{i,-1}}{\bar{\sigma}^{2}} \\
\Psi_{\sigma^{2}, i}\left(\bar{r}, \bar{\sigma}^{2}\right) & =-\frac{T-1}{2 \bar{\sigma}^{2}}+\frac{\varepsilon_{i}^{\prime} Q \varepsilon_{i}}{2 \bar{\sigma}^{4}}
\end{aligned}
$$

When $\bar{\sigma}^{2}$ is known we only consider $\Psi_{r, i}$ and substitute $\bar{\sigma}^{2}$ for $\sigma^{2}$. For the sake of convenience we treat the $y_{i, 0}{ }^{\prime} s$ as fixed numbers. This is a stronger assumption than $\varepsilon_{i} \mid \check{\mu}_{i}, y_{i, 0} \sim$ $N\left(0, \sigma^{2} I\right)$. This assumption could be relaxed at the cost of making additional assumptions 
with respect to the distribution of the $y_{i, 0}{ }^{\prime} s$.

First step: to show that

$$
\begin{aligned}
E \Psi_{r, i}\left(\bar{r}, \bar{\sigma}^{2}\right) & =0 \\
E \Psi_{\sigma^{2}, i}\left(\bar{r}, \bar{\sigma}^{2}\right) & =0
\end{aligned}
$$

Proof of the first step:

$$
\begin{aligned}
E \Psi_{r, i}\left(\bar{r}, \bar{\sigma}^{2}\right) & =\beta^{\prime}(\bar{r})+\frac{1}{\bar{\sigma}^{2}} E\left(\varepsilon_{i}^{\prime} Q y_{i,-1}\right)=\beta^{\prime}(\bar{r})+\frac{1}{\bar{\sigma}^{2}} E\left(\varepsilon_{i}^{\prime} Q \check{P}(\bar{r}) \varepsilon_{i}\right) \\
& =\beta^{\prime}(\bar{r})+\operatorname{tr}(Q \check{P}(\bar{r}))=\beta^{\prime}(\bar{r})-\frac{\iota^{\prime} \check{P}(\bar{r}) \iota}{T}=\beta^{\prime}(\bar{r})-\beta^{\prime}(\bar{r})=0 \\
E \Psi_{\sigma^{2}, i}\left(\bar{r}, \bar{\sigma}^{2}\right) & =-\frac{T-1}{2 \bar{\sigma}^{2}}+\frac{\bar{\sigma}^{2}}{2 \bar{\sigma}^{4}} \operatorname{tr}(Q)=0
\end{aligned}
$$

where $\check{P}(=\check{P}(r))$ is given in $(26)$.

Second step: to show that

$$
\begin{aligned}
\lim _{N \rightarrow \infty} \operatorname{Var}\left(\frac{1}{N} \sum_{i=1}^{N} \Psi_{r, i}\left(\bar{r}, \bar{\sigma}^{2}\right)\right) & =0 \\
\lim _{N \rightarrow \infty} \operatorname{Var}\left(\frac{1}{N} \sum_{i=1}^{N} \Psi_{\sigma^{2}, i}\left(\bar{r}, \bar{\sigma}^{2}\right)\right) & =0
\end{aligned}
$$

Proof of the second step:

$\lim _{N \rightarrow \infty} \operatorname{Var}\left(\frac{1}{N} \sum_{i=1}^{N} \Psi_{r, i}\left(\bar{r}, \bar{\sigma}^{2}\right)\right)=0$, and $\lim _{N \rightarrow \infty} \operatorname{Var}\left(\frac{1}{N} \sum_{i=1}^{N} \Psi_{\sigma^{2}, i}\left(\bar{r}, \bar{\sigma}^{2}\right)\right)=0$ follow from the fact that $\Psi_{r, i}\left(\bar{r}, \bar{\sigma}^{2}\right)$ and $\Psi_{\sigma^{2}, i}\left(\bar{r}, \bar{\sigma}^{2}\right)$ are independent across individuals, that $\left(\varepsilon_{i}^{\prime} Q \varepsilon_{i}\right) / \bar{\sigma}^{2} \sim \chi^{2}(T-1)$, and that $\left(\varepsilon_{i}^{\prime} Q \check{P} \varepsilon_{i}\right) / \bar{\sigma}^{2}$ has a finite variance.

Third step: to show that there exist numbers $A_{1}<0$ and $A_{2}<0$ such that

$$
\begin{aligned}
\lim _{N \rightarrow \infty} P\left(\frac{1}{N} \sum_{i=1}^{N} \frac{d \Psi_{r, i}\left(\bar{r}, \bar{\sigma}^{2}\right)}{d r}<A_{1}\right) & =1 \\
\lim _{N \rightarrow \infty} P\left(\frac{1}{N} \sum_{i=1}^{N} \frac{d \Psi_{\sigma^{2}, i}\left(\bar{r}, \bar{\sigma}^{2}\right)}{d \sigma^{2}}<A_{2}\right) & =1
\end{aligned}
$$

Proof of the third step:

$$
\begin{aligned}
\frac{d \Psi_{r, i}\left(r, \sigma^{2}\right)}{d r} & =\beta^{\prime \prime}(r)-\frac{y_{i,-1}^{\prime} Q y_{i,-1}}{\sigma^{2}} \\
\frac{d \Psi_{\sigma^{2}, i}\left(r, \sigma^{2}\right)}{d \sigma^{2}} & =\frac{T-1}{2 \sigma^{4}}-\frac{1}{\sigma^{6}}\left(y_{i}-r y_{i,-1}\right)^{\prime} Q\left(y_{i}-r y_{i,-1}\right)
\end{aligned}
$$




$$
\begin{aligned}
p \lim _{N \rightarrow \infty} \frac{1}{N} \sum_{i=1}^{N} \frac{d \Psi_{r, i}\left(\bar{r}, \bar{\sigma}^{2}\right)}{d r} & =\beta^{\prime \prime}(\bar{r})-\lim _{N \rightarrow \infty} \frac{1}{N} \sum_{i=1}^{N} \frac{E\left(y_{i,-1}^{\prime} Q y_{i,-1}\right)}{\bar{\sigma}^{2}}<0 \\
p \lim _{N \rightarrow \infty} \frac{1}{N} \sum_{i=1}^{N} \frac{d \Psi_{\sigma^{2}, i}\left(\bar{r}, \bar{\sigma}^{2}\right)}{d \sigma^{2}} & =-\frac{T-1}{2 \bar{\sigma}^{4}}<0
\end{aligned}
$$

For the first inequality we refer to the Expected Modified Hessian in (28) and the discussion below (28).

Last step: to show that there exist functions $H_{1}\left(y_{i}, y_{i, 0}\right)$, and $H_{2}\left(y_{i}, y_{i, 0}\right)$ such that

$$
\begin{aligned}
\left|\frac{d^{2} \Psi_{r, i}\left(\bar{r}, \bar{\sigma}^{2}\right)}{d r^{2}}\right| & =\left|\beta^{\prime \prime \prime}(\bar{r})\right|<H_{1}\left(y_{i}, y_{i, 0}\right) \\
\left|\frac{d^{2} \Psi_{\sigma^{2}, i}\left(\bar{r}, \bar{\sigma}^{2}\right)}{d\left(\sigma^{2}\right)^{2}}\right| & =\left|-\frac{T-1}{\bar{\sigma}^{6}}+\frac{3}{\bar{\sigma}^{8}}\left(y_{i}-\bar{r} y_{i,-1}\right)^{\prime} Q\left(y_{i}-\bar{r} y_{i,-1}\right)\right| \\
& <H_{2}\left(y_{i}, y_{i, 0}\right)
\end{aligned}
$$

and there exist numbers $M_{1}$ and $M_{2}$ which do not depend on $\bar{r}, \bar{\sigma}^{2}$ such that

$$
\begin{aligned}
& \lim _{N \rightarrow \infty} P\left(\frac{1}{N} \sum_{i=1}^{N} E\left(H_{1}\left(y_{i}, y_{i, 0}\right)\right)<M_{1}\right)=1 \\
& \lim _{N \rightarrow \infty} P\left(\frac{1}{N} \sum_{i=1}^{N} E\left(H_{2}\left(y_{i}, y_{i, 0}\right)\right)<M_{2}\right)=1
\end{aligned}
$$

Proof of the fourth step:

$$
\exists H_{1} \text { such that }\left|\beta^{\prime \prime \prime}(\bar{r})\right|<H_{1}
$$

Next we define $H_{2}\left(y_{i}, y_{i, 0}\right)$ :

$$
\begin{aligned}
\mid- & \frac{T-1}{\bar{\sigma}^{6}}+\frac{3}{\bar{\sigma}^{8}}\left(y_{i}-\bar{r} y_{i,-1}\right)^{\prime} Q\left(y_{i}-\bar{r} y_{i,-1}\right) \mid \\
& <\frac{2(T-1)}{\bar{\sigma}^{6}}+\frac{4}{\bar{\sigma}^{8}} \varepsilon_{i}^{\prime} Q \varepsilon_{i} \\
& <\frac{2(T-1)}{\delta^{6}}+\frac{4}{\delta^{6} \bar{\sigma}^{2}} \varepsilon_{i}^{\prime} Q \varepsilon_{i}
\end{aligned}
$$

where we have used that $-1<\bar{r} \leq 1$ and that $\bar{\sigma}^{2}>\delta^{2}>0$ and where $\delta$ is an arbitrarily small number. Therefore we define

$$
H_{2}\left(y_{i}, y_{i, 0}, \delta\right) \equiv \frac{2(T-1)}{\delta^{6}}+\frac{4}{\delta^{6} \bar{\sigma}^{2}} \varepsilon_{i}^{\prime} Q \varepsilon_{i}
$$


Indeed there exist numbers $M_{1}$ and $M_{2}(\delta)$ such that

$$
\begin{aligned}
\lim _{N \rightarrow \infty} P\left(\frac{1}{N} \sum_{i=1}^{N} E\left(H_{1}\right)<M_{1}\right) & =1 \\
\lim _{N \rightarrow \infty} P\left(\frac{1}{N} \sum_{i=1}^{N} E\left(H_{2}\left(y_{i}, y_{i, 0}, \delta\right)\right)<M_{2}(\delta)\right) & =1
\end{aligned}
$$

So far we have shown (analytically) that $E \log p\left(r, \sigma^{2} ; \bar{r}, \bar{\sigma}^{2}\right)$ is maximized at the true values $\bar{r}, \bar{\sigma}^{2}$, and that one of the roots of $\frac{1}{N} \sum_{i=1}^{N} \log p_{i}\left(r, \sigma^{2}\right)$ is a consistent estimator of $(\bar{r}$ $\left.\bar{\sigma}^{2}\right)^{\prime}$. We will now address the question how the consistent root can be selected.

We will first investigate whether $E \log p\left(r, \sigma^{2} ; \bar{r}, \bar{\sigma}^{2}\right)$ attains a unique global maximum at the true values for arbitrary $\left(\bar{r} \bar{\sigma}^{2}\right) \in(-1,1) \times(0, \infty), \bar{\mu}_{i}^{\prime} s, y_{i, 0}^{\prime} s$, and $T$. As $p\left(r, \sigma^{2}\right)$ is not a product of proper densities, we cannot use the standard 'Jensen inequality' argument to prove that the $E \log p\left(r, \sigma^{2} ; \bar{r}, \bar{\sigma}^{2}\right)$ has a unique global maximum at $\left(\bar{r} \bar{\sigma}^{2}\right)^{\prime}$. Instead we will take a closer look at graphs of $E \log p\left(r, \sigma^{2} ; \bar{r}, \bar{\sigma}^{2}\right)$.

First, we observe that $E \log p\left(r, \sigma^{2} ; \bar{r}, \bar{\sigma}^{2}\right)$ only depends on the $\overline{\breve{\mu}}_{i}^{\prime} s$ and the $y_{i, 0}^{\prime} s$ through

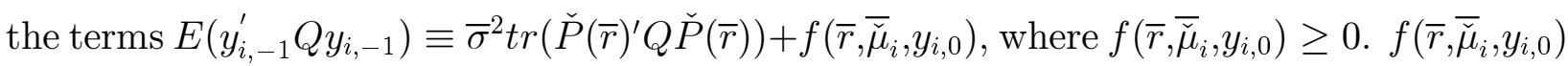
$=0$ if, for example, $\bar{\mu}_{i}=0$ and $y_{i, 0}=0$. Now, if $E \log p\left(r, \sigma^{2} ; \bar{r}, \bar{\sigma}^{2}\right)$ would have a unique global maximum at $\left(\bar{r} \bar{\sigma}^{2}\right)^{\prime}$ (taking boundery maxima into account) when $f\left(\bar{r}, \bar{\mu}_{i}, y_{i, 0}\right)=0 \forall$ $i$, this would be the case a fortiori when $f\left(\bar{r}, \bar{\mu}_{i}, y_{i, 0}\right)>0$ for some or all i. Therefore, we will

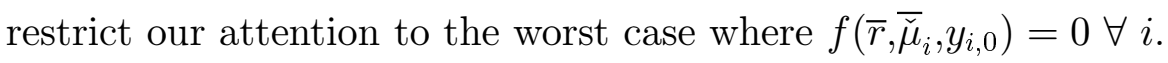

When studying the graphs we will distinguish two cases. In the first case $\bar{r}$ is unknown and $\bar{\sigma}^{2}$ is known, in the second case both $\bar{r}$ and $\bar{\sigma}^{2}$ are unknown.

In the first case (where $\bar{\sigma}^{2}$ is known) a 4-dimensional graph (with axes for $r, \bar{r}, \bar{\sigma}^{2}$, and $\left.E \log p\left(r, \bar{\sigma}^{2} ; \bar{r}, \bar{\sigma}^{2}\right)\right)$ would summarize all the information on $E \log p\left(r, \bar{\sigma}^{2} ; \bar{r}, \bar{\sigma}^{2}\right)$ for all true values $\left(\bar{r} \bar{\sigma}^{2}\right) \in(-1,1) \times(0, \infty)$ and for a particular value of $T$. Obviously, we cannot look at 4-dimensional graphs. However, we can reduce the dimension of the graphs without loss of generality by observing that the shape of the graphs of $E \log p\left(r, \bar{\sigma}^{2} ; \bar{r}, \bar{\sigma}^{2}\right)$ is always the same (up to a constant) whatever the value of $\bar{\sigma}^{2}$. Therefore we can take $\bar{\sigma}^{2}=1$. We studied 3D-graphs of $E \log p(r, 1 ; \bar{r}, 1)$ for several values of $T$, that is for all values of $T \leq 15, T=25,50,100$. For each value of $T$ we found that $E \log p(r, 1 ; \bar{r}, 1)$ has a unique maximum at $r=\bar{r}$ on the interval $-1<r<1$, for all $\bar{r}$ with $-1<\bar{r}<1$. It is 
easy to check whether $E \log p(r, 1 ; \bar{r}, 1)$ has a unique maximum at $r=\bar{r}$ for other values of $T$ using Maple and the program of five lines given at the end of this proof ${ }^{35}$.

In the second case (where $\bar{\sigma}^{2}$ is unknown) a 5-dimensional graph would contain all the information on $E \log p\left(r, \sigma^{2} ; \bar{r}, \bar{\sigma}^{2}\right)$. However, we can reduce the dimension of the graphs again. We observe that when $f\left(\bar{r}, \overline{\breve{\mu}}_{i}, y_{i, 0}\right)=0 \forall i$ the "expected modified likelihood equation" for $\sigma^{2}$ has a unique solution for $\sigma^{2}$ which is proportional to $\bar{\sigma}^{2}$. This allows us to concentrate $E \log p\left(r, \sigma^{2} ; \bar{r}, \bar{\sigma}^{2}\right)$. Furthermore, we can restrict our attention to the case $\bar{\sigma}^{2}=1$. Therefore we can again study 3D-graphs (with axes for $r, \bar{r}$, and $E \log p_{C}(r ; \bar{r})$ ) for various values of $T$. Although the graphs we looked at had unique (interior) maxima at $r=\bar{r}, \forall \bar{r}$ with $-1<\bar{r}<1$, we also found higher values for $E \log p_{C}(r ; \bar{r})$ than these (interior) maxima for $r$ close to 1 , that is we found a boundery maximum at $r=1$, e.g. when $T=5$ and $\bar{r}=0.7$. As a consequence, we cannot rule out the existence of multiple (interior) maxima on the interval $-1<r<1$ for a particular value of $\bar{r}$ with $-1<\bar{r}<1$ when $f\left(\bar{r}, \bar{\mu}_{i}, y_{i, 0}\right)>0$ for some or all $i$, and what is worse we cannot rule out that the maximum at $r=\bar{r}$ is lower than another interior maximum when $f\left(\bar{r}, \bar{\mu}_{i}, y_{i, 0}\right)>0$ for some or all $i$. Therefore the question is still how can we identify the maximum corresponding to the true value of $r$. We will show that for any values of $f\left(\bar{r}, \bar{\mu}_{i}, y_{i, 0}\right) \geq 0, E \log p_{C}(r ; \bar{r})$ has no maximum on $(-1, \bar{r})$, when $-1<\bar{r}<1$, and therefore the maximum in the interval $(-1,1)$ which is closest to -1 is a consistent estimator of $\bar{r}$. The proof is as follows:

Up to irrelevant constants $E \log p_{C}(r ; \bar{r})$ is equal to $E \log \check{p}_{C}(r ; \bar{r})=-\frac{T-1}{2} \log z(\bar{r}, r)+g(r)$ where $g(r)=\frac{1}{T} \sum_{t=1}^{T-1} \frac{T-t}{t} r^{t}, z(\bar{r}, r)=\frac{T-1}{2}+\frac{1}{2}(r-\bar{r})^{2}\left(q(\bar{r})+\frac{1}{N} \sum_{i=1}^{N} f\left(\bar{r}, \bar{\mu}_{i}, y_{i, 0}\right)\right)+(r-\bar{r}) h(\bar{r})$, with $h(\bar{r})=\frac{1}{T} \sum_{t=1}^{T-1}(T-t) \bar{r}^{t-1}$, and $q(\bar{r})=\left(\sum_{t=0}^{T-2} \sum_{p=0}^{t} \bar{r}^{2 p}\right)-\frac{1}{T} \sum_{t=0}^{T-2}\left(\sum_{p=0}^{t} \bar{r}^{p}\right)^{2}$.

We will show that $\frac{\partial}{\partial r} E \log \check{p}_{C}(r ; \bar{r}) \geq 0$ for any values of $f\left(\bar{r}, \bar{\mu}_{i}, y_{i, 0}\right) \geq 0$ when $-1<r<$ $\bar{r}<1$. Let $f=\frac{1}{N} \sum_{i=1}^{N} f\left(\bar{r}, \bar{\mu}_{i}, y_{i, 0}\right)$ and let $k(r, f ; \bar{r})=\frac{T-1}{2}+\frac{1}{2}(r-\bar{r})^{2}(q(\bar{r})+f)+(r-\bar{r}) h(\bar{r})$. First it can be seen from the graphs that $\frac{\partial}{\partial r} E \log \check{p}_{C}(r ; \bar{r}) \geq 0$ when $f=0$ and $-1<r<$ $\bar{r}<1$. Now, $E \log \check{p}_{C}(r ; \bar{r})=-\frac{T-1}{2} \log k(r, 0 ; \bar{r})+g(r)-\frac{T-1}{2} \log [k(r, f ; \bar{r}) / k(r, 0 ; \bar{r})]$. Moreover,

\footnotetext{
${ }^{35}$ When $0 \leq \bar{r}<1$, it is easily shown that the expected modified likelihood equation for $r$, $\beta^{\prime}(r)-\beta^{\prime}(\bar{r})+(\bar{r}-r) \operatorname{tr}\left(\check{P}(\bar{r})^{\prime} Q \check{P}(\bar{r})\right)=0$, has two solutions: one corresponding to a maximum, $\bar{r},\left(\beta^{\prime \prime}(\bar{r})-\operatorname{tr}\left(\check{P}(\bar{r})^{\prime} Q \check{P}(\bar{r})\right) \leq 0\right)$ and one corresponding to a minimum. It follows that in this case $E \log p\left(r, \bar{\sigma}^{2} ; \bar{r}, \bar{\sigma}^{2}\right)$ has a unique maximum at $r=\bar{r}$.
} 
$\frac{\partial}{\partial r} \log [k(r, f ; \bar{r}) / k(r, 0 ; \bar{r})]=\left\{k(r, 0 ; \bar{r})\left[\frac{\partial}{\partial r} k(r, f ; \bar{r})\right]-k(r, f ; \bar{r})\left[\frac{\partial}{\partial r} k(r, 0 ; \bar{r})\right]\right\} /[k(r, 0 ; \bar{r}) k(r, f ; \bar{r})]$. Clearly, $k(r, f ; \bar{r})>0, \forall f \geq 0$. In addition, $k(r, 0 ; \bar{r})\left[\frac{\partial}{\partial r} k(r, f ; \bar{r})\right]-k(r, f ; \bar{r})\left[\frac{\partial}{\partial r} k(r, 0 ; \bar{r})\right]=$ $f(r-\bar{r}) k(r, 0 ; \bar{r})-0.5 f(r-\bar{r})^{2}[(r-\bar{r}) q(\bar{r})+h(\bar{r})]=0.5(T-1) f(r-\bar{r})+0.5 f(r-\bar{r})^{2} h(\bar{r})=$ $f(r-\bar{r})[0.5(T-1)+0.5(r-\bar{r}) h(\bar{r})] \leq 0$ for all $-1<r \leq \bar{r}<1$, because $|h(\bar{r})| \leq 0.5(T-1)$ and $|r-\bar{r}| \leq 2$. It follows that $\frac{\partial}{\partial r} E \log \check{p}_{C}(r ; \bar{r}) \geq 0, \forall f \geq 0$ when $-1<r<\bar{r}<1$, and therefore $\forall f \geq 0, E \log p_{C}(r ; \bar{r})$ will have no local maximum on the interval $(-1, \bar{r})$.

In case the concentrated modified likelihood function has more than one maximum on the interval $(-1,1)$, we could also follow Amemiya (1986). He suggests to compute a preliminary estimate using a consistent estimator and to select the maximum that is closest to the preliminary estimate (see comments after his theorem 4.1.2). The estimate for $\sigma^{2}$ is obtained by substituting the estimate for $r$ in the modified likelihood equation for $\sigma^{2}$.

\section{COMMENTS ON THE GRAPHS ON PAGES 53 UNTIL 55:}

In the graphs $r$ is the true value of the parameter ( $\bar{r}$ in the paper) and $s$ is the unknown parameter value ( $r$ in the paper).

The first four graphs on page 53 correspond to the case where $\bar{\sigma}^{2}$ is known. In this particular case $T=5$. From the first graph we see that there is a unique maximum at $r=\bar{r}$ ("s $=r$ ") for all $\bar{r}$ with $-1<\bar{r}<1$. The other three graphs are added just to be sure. For the other values of $T$ that we considered we obtained similar graphs (showing unique maxima).

The second series of graphs on page 55 correspond to the case where both $\bar{r}$ and $\bar{\sigma}^{2}$ are unknown. The graphs are presented for $T=5$ again. The top graph again shows maxima at $r=\bar{r}(" s=r ")$. However, when we look at a cross section of this graph at $\bar{r}=0.7$ (the third and fourth graphs on page 54), we find that, although there is a unique interior maximum at $r=0.7$ (" $s=0.7 ")$, the boundery maximum at $r=1(" s=1 ")$ is higher than the interior maximum.

Program for Maple for checking uniqueness of maximum when $\bar{\sigma}^{2}$ is known and $T$ is given:

$$
\begin{aligned}
& g(s)=\frac{1}{T} \sum_{t=1}^{T-1} \frac{T-t}{t} s^{t} \\
& h(s)=\frac{1}{T} \sum_{t=1}^{T-1}(T-t) s^{t-1} \\
& q(r)=\left(\sum_{t=0}^{T-2} \sum_{p=0}^{t} r^{2 p}\right)-\frac{1}{T} \sum_{t=0}^{T-2}\left(\sum_{p=0}^{t} r^{p}\right)^{2} \\
& v(r, s)=g(s)-\frac{1}{2}(s-r)^{2} q(r)-(s-r) h(r)
\end{aligned}
$$




$$
T=5
$$

$v(r, s)$
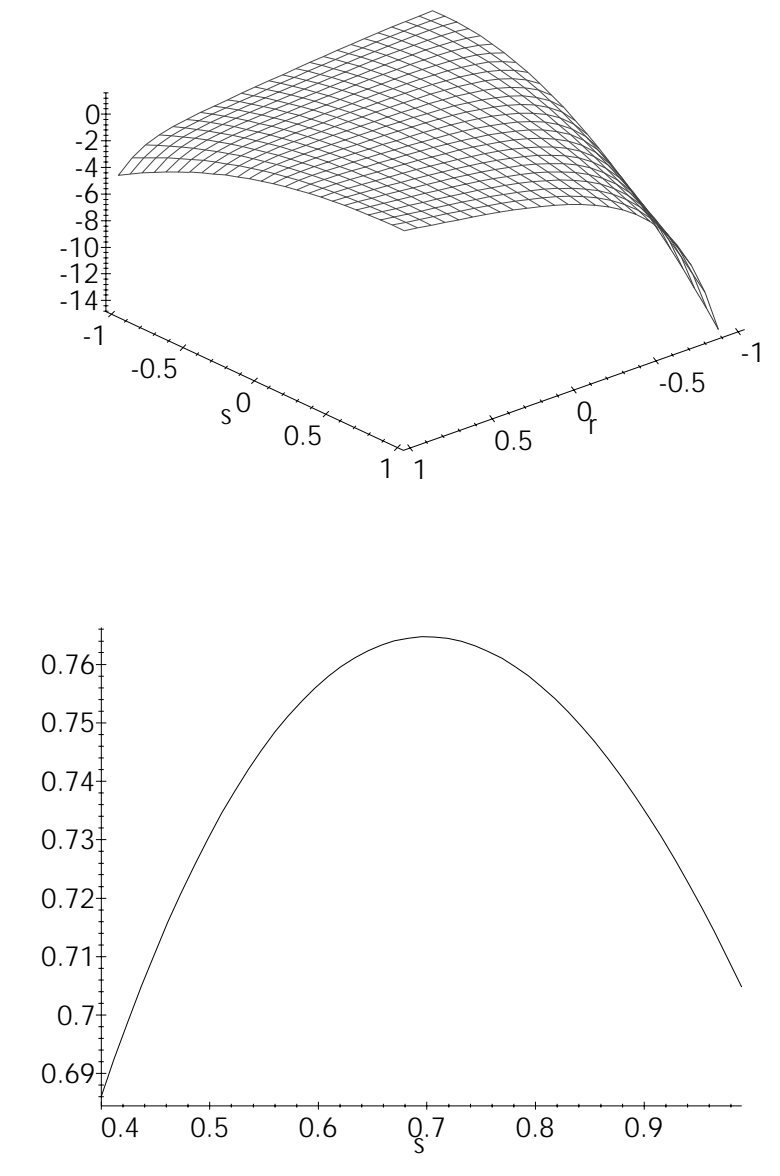

$v(0.7, s)$

$v(0.2, s)$

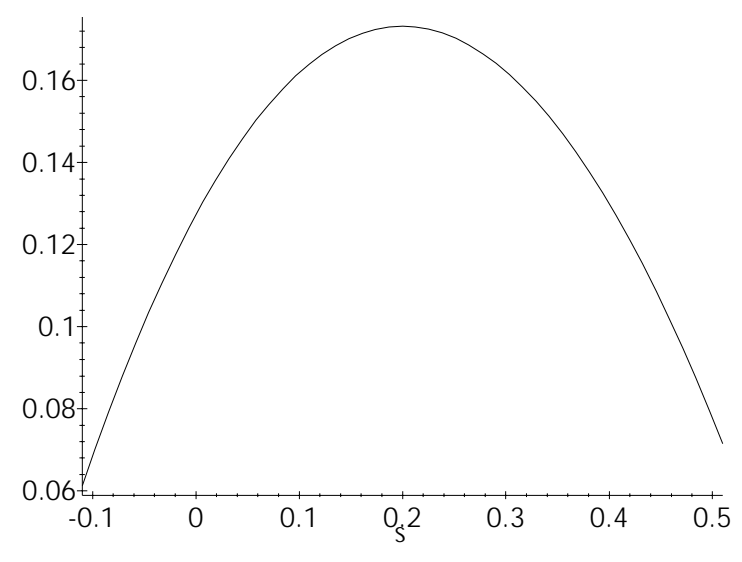




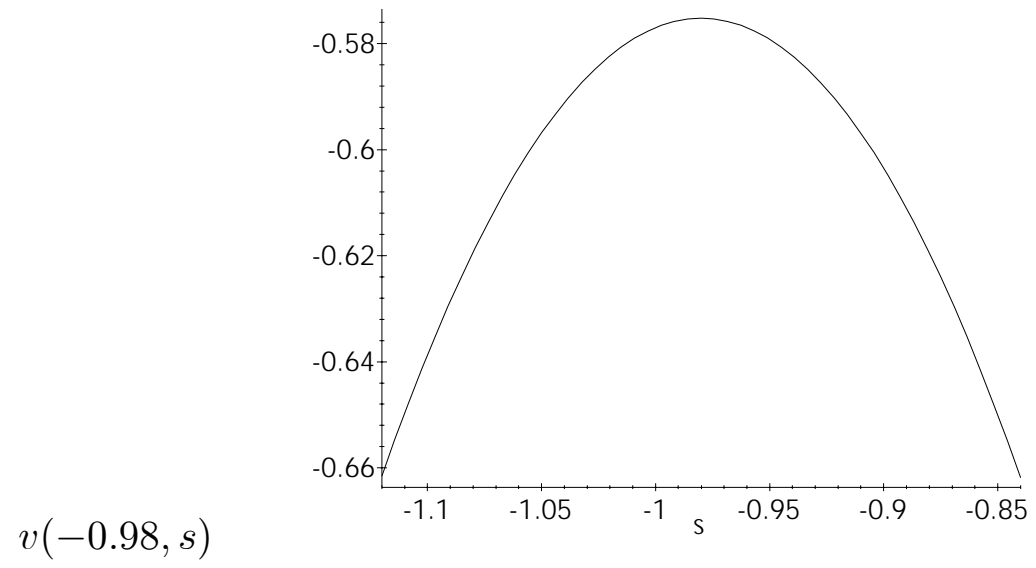

Program for Maple for checking uniqueness of maximum when $\bar{\sigma}^{2}$ is unknown for a given value of $T$

$$
\begin{aligned}
& g(s)=\frac{1}{T} \sum_{t=1}^{T-1} \frac{T-t}{t} s^{t} \\
& h(s)=\frac{1}{T} \sum_{t=1}^{T-1}(T-t) s^{t-1} \\
& q(r)=\left(\sum_{t=0}^{T-2} \sum_{p=0}^{t} r^{2 p}\right)-\frac{1}{T} \sum_{t=0}^{T-2}\left(\sum_{p=0}^{t} r^{p}\right)^{2} \\
& z(r, s)=\frac{T-1}{2}+\frac{1}{2}(s-r)^{2} q(r)+(s-r) h(r) \\
& w(r, s)=-\frac{T-1}{2} \log z(r, s)+g(s) \\
& T=5
\end{aligned}
$$

$w(r, s)$

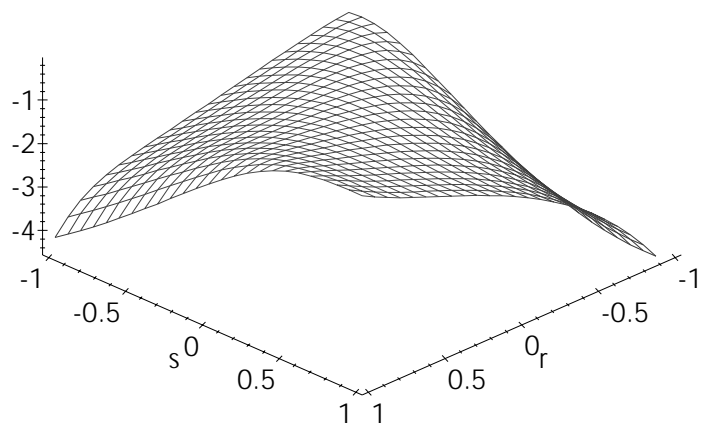



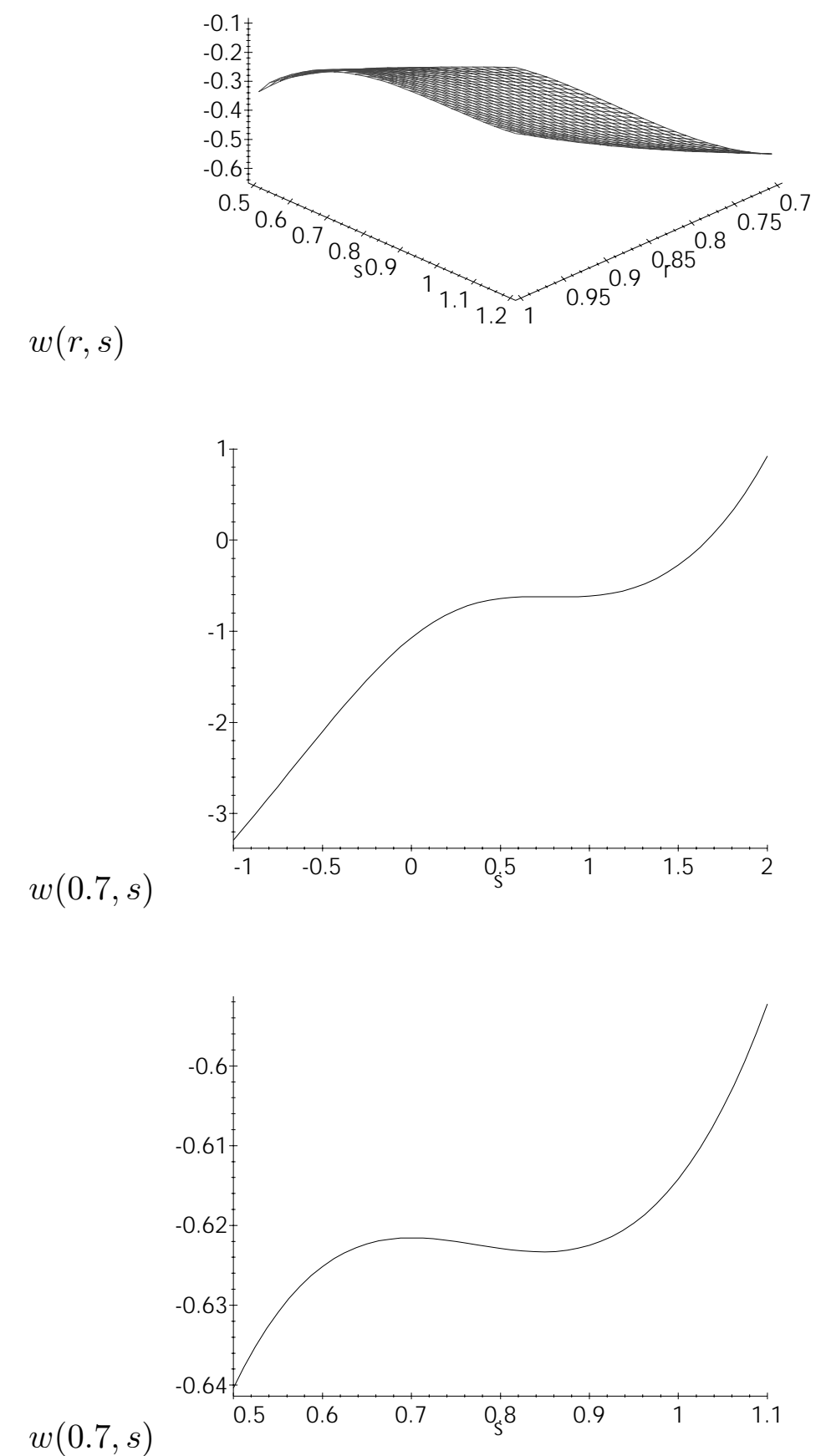


\section{B.2 Proof of theorem 5 (OCGMM is more efficient than MMLE):}

When $T=2$, the OCGMM exploits only two moment conditions: $E\left(m_{1,2}(r)\right)=0$, and $E\left(m_{2,2}(r)\right)=0$, where $m_{1,2}(r)=y_{i, 0} \Delta \varepsilon_{i, 2}$ and $m_{2,2}(r)=\left(y_{i, 2}-r y_{i, 1}\right)^{2}-\left(y_{i, 1}-r y_{i, 0}\right)^{2}$. Let $m(r)=\left(m_{1,2}(r) m_{2,2}(r)\right)^{\prime}$.

We have to show that the Optimal Conditional GMM estimator based on $E(m(r))=0$, $\widehat{r}_{O C G M M}$, is more efficient than the Modified ML estimator, $\widehat{r}_{M M L}$, when the $\left\{y_{i, t}\right\}$ are generated by stationary ARFE(1) models with normally distributed errors.

Now $E\left(\frac{d m}{d r}\right)=\left[\begin{array}{c}\frac{s}{1+r} \\ 0\end{array}\right]$, and

$E\left(m m^{\prime}\right)=\left[\begin{array}{ll}2 \sigma^{2}\left(a+\frac{\sigma^{2}}{1-r^{2}}\right) & 4 \sigma^{2}(1-r) a \\ 4 \sigma^{2}(1-r) a & 4 \sigma^{4}+8 \sigma^{2}(1-r)^{2} a\end{array}\right]$, where $a=\frac{1}{(1-r)^{2}} \frac{1}{N} \sum_{i=1}^{N} \check{\mu}_{i}^{2}$.

We find that $\operatorname{Avar}\left(\widehat{r}_{O C G M M}\right)=\frac{2\left(3 a+a r^{2}+\sigma^{2}-4 a r\right)(1+r)}{(1-r)\left(\sigma^{2}+2 a-4 a r+2 a r^{2}\right)}$.

We will now derive the asymptotic variance of the Modified ML estimator. It is easily verified that $\frac{E\left(y_{i,-1}^{\prime} Q y_{i,-1}\right)}{\sigma^{2}}=\frac{1}{1+r} ; \beta(r)=\frac{1}{2} r$; and $\operatorname{tr}(Q \check{P} Q \check{P})=\frac{1}{4}$.

Therefore, the expected Hessian equals

$$
E M H=\left(\begin{array}{cc}
\beta^{\prime \prime}(r)-\frac{1}{N} \sum_{i=1}^{N} \frac{E\left(y_{i,-1}^{\prime} Q y_{i,-1}\right)}{\sigma^{2}} & \frac{\beta^{\prime}(r)}{\sigma^{2}} \\
\frac{\beta^{\prime}(r)}{\sigma^{2}} & -\frac{T-1}{2 \sigma^{4}}
\end{array}\right)=\left(\begin{array}{cc}
-\frac{1}{1+r} & \frac{1}{2 \sigma^{2}} \\
\frac{1}{2 \sigma^{2}} & -\frac{1}{2 \sigma^{4}}
\end{array}\right)
$$

and the modified information matrix equals

$$
M I M=\left(\begin{array}{cc}
t r(Q \check{P} Q \check{P})+\frac{1}{N} \sum_{i=1}^{N} \frac{E\left(y_{i,-1}^{\prime} Q y_{i,-1}\right)}{\sigma^{2}} & -\frac{\beta^{\prime}(r)}{\sigma^{2}} \\
-\frac{\beta^{\prime}(r)}{\sigma^{2}} & \frac{T-1}{2 \sigma^{4}}
\end{array}\right)=\left(\begin{array}{cc}
\frac{1}{4}+\frac{1}{1+r} & -\frac{1}{2 \sigma^{2}} \\
-\frac{1}{2 \sigma^{2}} & \frac{1}{2 \sigma^{4}}
\end{array}\right)
$$

We obtain $(E M H)^{-1} M I M(E M H)^{-1}=\left(\begin{array}{cc}-\frac{-3+r}{(-1+r)^{2}}(1+r) & -\frac{-3+r}{(-1+r)^{2}} \sigma^{2}(1+r) \\ -\frac{-3+r}{(-1+r)^{2}} \sigma^{2}(1+r) & \sigma^{4} \frac{5-2 r+r^{2}}{(-1+r)^{2}}\end{array}\right)$, and $\operatorname{Avar}\left(\widehat{r}_{M M L}\right)=-\frac{-3+r}{(-1+r)^{2}}(1+r)$.

Finally, $\operatorname{Avar}\left(\widehat{r}_{M M L}\right)-\operatorname{Avar}\left(\widehat{r}_{O C G M M}\right)=\sigma^{2} \frac{(1+r)^{2}}{(1-r)^{2}\left(\sigma^{2}+2 a-4 a r+2 a r^{2}\right)}>0$ since $2 a-4 a r+$ $2 a r^{2}=2(1-r)^{2} a>0$. 


\section{Proofs of Results in SeCtion 4}

\section{C.1 Proof of theorem 9:}

Note that $T=3$, and $-1<r<1$. Recall that $\theta=\left(\begin{array}{ll}r^{\prime} & \sigma^{2}\end{array}\right)^{\prime}$.

Remember that the Linear GMM estimator exploits all the moment conditions that are implied by covariance stationarity. Therefore the moment conditions in $E\left(\operatorname{vech}\left(Z_{i}\right)\right)-$ $\operatorname{vech}(\Omega(\theta))=0$ are a subset of the moment conditions that are exploited by the Linear GMM estimator.

Under normality the covariance matrix of $f(\theta)=\left(y_{i, 1} \Delta \varepsilon_{i, 3} \quad\left(\operatorname{vech}\left(Z_{i}\right)-\operatorname{vech}(\Omega(\theta))\right)^{\prime}\right)^{\prime}$ equals $E\left(f(\theta) f(\theta)^{\prime}\right)=\left[\begin{array}{cccc}a & \frac{2}{1+r} \sigma^{4} & -\frac{2(1-r)}{1+r} \sigma^{4} & -\frac{2 r(2-r)}{1+r} \sigma^{4} \\ \frac{2}{1+r} \sigma^{4} & \frac{8}{(1+r)^{2}} \sigma^{4} & -4 \frac{1-r}{(1+r)^{2}} \sigma^{4} & 2 \frac{(1-r)^{2}}{(1+r)^{2}} \sigma^{4} \\ -\frac{2(1-r)}{1+r} \sigma^{4} & -4 \frac{1-r}{(1+r)^{2}} \sigma^{4} & \frac{5-2 r+r^{2}}{(1+r)^{2}} \sigma^{4} & -4 \frac{1-r}{(1+r)^{2}} \sigma^{4} \\ -\frac{2 r(2-r)}{1+r} \sigma^{4} & 2 \frac{(1-r)^{2}}{(1+r)^{2}} \sigma^{4} & -4 \frac{1-r}{(1+r)^{2}} \sigma^{4} & \frac{8}{(1+r)^{2}} \sigma^{4}\end{array}\right]$ with $a=\frac{1}{N} \sum_{i=1}^{N} \operatorname{Var}\left(y_{i, 1} \Delta \varepsilon_{i, 3}\right)=\frac{2 \sigma^{4}}{1-r^{2}}+\frac{2}{N} \sum_{i=1}^{N} \mu_{i}^{2} \sigma^{2}=\frac{2 \sigma^{4}}{1-r^{2}}+\frac{2}{N} \sum_{i=1}^{N} b_{i} \sigma^{4}$, where $b_{i}=\frac{\mu_{i}^{2}}{\sigma^{2}}$.

Note that $y_{i, 1} \Delta \varepsilon_{i, 3}=y_{i, 1}\left(\Delta y_{i, 3}-r \Delta y_{i, 2}\right)$. Then we have $E\left(\frac{d f}{d \theta^{\prime}}\right)^{\prime}=\left[\begin{array}{cccc}\frac{1}{1+r} \sigma^{2} & \frac{2}{(1+r)^{2}} \sigma^{2} & -\frac{2}{(1+r)^{2}} \sigma^{2} & \frac{2}{(1+r)^{2}} \sigma^{2} \\ 0 & -\frac{2}{1+r} & \frac{1-r}{1+r} & -\frac{2}{1+r}\end{array}\right]$.

Let $\widehat{r}_{G M M^{+}}$denote the Optimal GMM estimator that is based on $E(f(\theta))=0$, and let $\widehat{r}_{O M D}$ denote the Optimal GMM estimator that is based on $E\left(\operatorname{vech}\left(Z_{i}\right)\right)-\operatorname{vech}(\Omega(\theta))=0$. We find that $\operatorname{Var}\left(\widehat{r}_{O M D}\right)=\frac{1}{4}(1+r)^{2}(r-3)^{2}$ and $\operatorname{Var}\left(\widehat{r}_{G M M^{+}}\right)=$ $\frac{1}{\sigma^{4} r^{4}+4 a r^{2}-6 \sigma^{4} r^{2}-8 a r-8 \sigma^{4} r+13 \sigma^{4}-12 a}(r-3)^{2}\left(a r^{2}-2 a r-3 a+4 \sigma^{4}\right)(1+r)^{2}$.

The difference between the variances equals

$\operatorname{Var}\left(\widehat{r}_{O M D}\right)-\operatorname{Var}\left(\widehat{r}_{G M M^{+}}\right)=\frac{1}{4}(1+r)^{5}(r-3)^{3} \frac{\sigma^{4}}{\sigma^{4} r^{4}+4 a r^{2}-6 \sigma^{4} r^{2}-8 a r-8 \sigma^{4} r+13 \sigma^{4}-12 a}$.

Substituting $\frac{2 \sigma^{4}}{1-r^{2}}+2 b \sigma^{4}$ for $a$ yields $\sigma^{4} r^{4}+4 a r^{2}-6 \sigma^{4} r^{2}-8 a r-8 \sigma^{4} r+13 \sigma^{4}-12 a=$ $\sigma^{4} r^{4}+4\left(\frac{2 \sigma^{4}}{1-r^{2}}+2 b \sigma^{4}\right) r^{2}-6 \sigma^{4} r^{2}-8\left(\frac{2 \sigma^{4}}{1-r^{2}}+2 b \sigma^{4}\right) r-8 \sigma^{4} r+13 \sigma^{4}-12\left(\frac{2 \sigma^{4}}{1-r^{2}}+2 b \sigma^{4}\right)=$ $\left(r^{5}-r^{4}-6 r^{3}+8 b r^{3}-2 r^{2}-24 b r^{2}-8 r b+13 r+11+24 b\right) \frac{\sigma^{4}}{-1+r}$.

Observe that $8 b r^{3}-24 b r^{2}-8 r b+24 b=8(-1+r)(r-3)(1+r) b \geq 0$, and that $r^{5}-r^{4}-6 r^{3}-2 r^{2}+13 r+11=(1+r)\left(r^{4}-2 r^{3}-4 r^{2}+2 r+11\right)>0$ since $r^{4}-2 r^{3}-4 r^{2}+2 r+11>0$.

It follows that $\sigma^{4} r^{4}+4 a r^{2}-6 \sigma^{4} r^{2}-8 a r-8 \sigma^{4} r+13 \sigma^{4}-12 a<0$.

We conclude that $\operatorname{Var}\left(\widehat{r}_{O M D}\right)>\operatorname{Var}\left(\widehat{r}_{G M M^{+}}\right)$. 


\section{References}

[1] Abowd, J., and D. Card, 1989, On the covariance structure of earnings and hours changes, Econometrica 57, 411-445.

[2] Ahn, S.C., and P. Schmidt, 1995, Efficient estimation of models for dynamic panel data, Journal of econometrics $68,5-28$.

[3] Ahn, S.C., and P. Schmidt, 1997, Efficient estimation of dynamic panel data models: alternative assumptions and simplified estimation, Journal of econometrics 76, 309-321.

[4] Amemiya, T., 1986, Advanced Econometrics, Harvard University Press, Cambridge, Massachusetts.

[5] Andersen, E.B., 1970, Asymptotic properties of conditional maximum-likelihood estimators, JRSS Series B 32, 283-301.

[6] Andersen, E.B., 1973, Conditional inference and models for measuring, Mentalhygiejnisk Forlag, Copenhagen.

[7] Anderson, T.W., and C. Hsiao, 1981, Estimation of dynamic models with error components, Journal of the American statistical association 76, 598-606.

[8] Anderson, T.W., and C. Hsiao, 1982, Formulation and estimation of dynamic models using panel data, Journal of econometrics 18, 47-82.

[9] Andrews, D.W.K., 1999, Estimation when a parameter is on a boundary, forthcoming in Econometrica.

[10] Arellano, M., 1990, Testing for autocorrelation in dynamic random effects models, Review of economic studies $57,127-134$.

[11] Arellano, M., and S. Bond, 1991, Some tests of specification for panel data: Monte Carlo evidence and an application to employment equations, Review of economic studies 58, 277-297. 
[12] Arellano, M., and O. Bover, 1995, Another look at the instrumental variable estimation of error-components models, Journal of econometrics 68, 29-51.

[13] Balestra, P., and M. Nerlove, 1966, Pooling cross-section and time series data in the estimation of a dynamic model: the demand for natural gas, Econometrica 34, 585-612.

[14] Blundell, R.W., and R.J. Smith, 1991, Initial conditions and efficient estimation in dynamic panel data models, Annales d'économie et de la statistique 20/21, 109-123.

[15] Breitung, J., and W. Meyer, 1994, Testing for unit roots using panel data: are wages on different bargaining levels cointegrated?, Applied economics 26, 353-361.

[16] Breusch, T., H. Qian, P. Schmidt, and D. Wyhowski, 1999, Redundancy of moment conditions, Journal of econometrics 91, 89-111.

[17] Chamberlain, G., 1980, Analysis of covariance with qualitative data, Review of economic studies 47, 225-238.

[18] Chamberlain, G., 1982, Multivariate regression models for panel data, Journal of econometrics 18, 5-46.

[19] Chamberlain, G., 1984, Panel data, in Handbook of econometrics, edited by Z. Griliches and M. Intrilligator, North Holland, Amsterdam.

[20] Cramér, H., 1946, Mathematical methods in statistics, Princeton University Press, Princeton, New Jersey.

[21] Crépon, B., F. Kramarz, and A. Trognon, 1997, Parameters of interest, nuisance parameters, and orthogonality conditions, Journal of econometrics 82, 135-156.

[22] Harris, R.D.F., and E. Tzavalis, 1999, Inference for unit roots in dynamic panels where the time dimension is fixed, Journal of econometrics 91, 201-226.

[23] Kiefer, N.M., 1980, Estimation of fixed effect models for time series of cross-sections with arbitrary intertemporal covariance, Journal of econometrics 14, 195-202. 
[24] Kiviet, J.F., 1995, On the bias, inconsistency, and efficiency of various estimators in dynamic panel data models, Journal of econometrics $68,53-78$.

[25] Kruiniger, H., 1998a, Conditional maximum likelihood estimation of dynamic panel data models, University College London Economics Paper 98-04.

[26] Kruiniger, H., 1998b, CML and GMM based unit root tests for dynamic panel data models with fixed effects, Agricultural Economic Research Institute LEI-DLO, The Hague, mimeographed.

[27] Kruiniger, H., 1999, CML and GMM estimation of dynamic panel data models with nonparametric cross-sectional heteroskedasticity, Agricultural Economic Research Institute LEI-DLO, The Hague, mimeographed.

[28] Kruiniger, H., 2000, GMM estimation of dynamic panel data models with persistent data, Queen Mary and Westfield College, University of London, working paper.

[29] Lancaster, T., 1997a (April), Consistent, likelihood-based, inference about short dynamic panels, mimeo, Brown University, Providence, Rhode Island.

[30] Lancaster, T., 1997b (December), Orthogonal parameters and panel data, working paper no. 97-32, Brown University, Providence, Rhode Island.

[31] Lancaster, T., and B. Lindenhovius, 1996, Biases in dynamic panel data models: a reconsideration (first revision), working paper no. 96-11, Brown University, Providence, Rhode Island.

[32] Lindley, D.V., and G.M. El-Sayyed, 1968, The Bayesian estimation of a linear functional relationship, JRSS Series B 30, 190-202.

[33] MaCurdy, T., 1981a, Multiple time series models applied to panel data, NBER working paper no. W0646, Cambridge, Massachusetts.

[34] MaCurdy, T., 1981b, Asymptotic properties of quasi-maximum likelihood estimators and test statistics, NBER technical working paper no. T0014, Cambridge, Massachusetts. 
[35] MaCurdy, T., 1982, The use of time series processes to model the time structure of earnings in a longitudinal data analysis, Journal of econometrics 18, 83-114.

[36] Magnus, J.R., 1978, Maximum likelihood estimation of the GLS model with unknown parameters in the disturbance covariance matrix, Journal of econometrics 7, 281-312, with corrigenda 10, 261.

[37] Magnus, J.R., and H. Neudecker, 1988, Matrix differential calculus with applications in statistics and econometrics, Wiley, Chicester, United Kingdom.

[38] Newey, W., 1990, Semiparametric efficiency bounds, Journal of applied econometrics 5, 99-136.

[39] Newey, W., and D. McFadden, 1994, Large sample estimation and hypothesis testing, Ch. 36 in Handbook of econometrics, vol. 4, R.F. Engle and D. McFadden eds., Elsevier, Amsterdam.

[40] Neyman, J., and E.L. Scott, 1948, Consistent estimates based on partially consistent observations, Econometrica 16, 1-32.

[41] Nickell, S., 1981, Biases in dynamic models with fixed effects, Econometrica 49, 14171426.

[42] Oberhofer, W., and J. Kmenta, 1974, A General Procedure for Obtaining Maximum Likelihood Estimates in Generalized Regression Models, Econometrica 42, 579-590.

[43] Phillips, P.C.B., and H. Moon, 1999, Linear Regression Limit Theory for Nonstationary Panel Data, Econometrica 67, 1057-1111.

[44] Rao, C.R., 1973, Linear statistical inference and its applications, 2nd edition, Wiley, New York.

[45] Rasch, G., 1961, On general laws and the meaning of measurement in psychology, Proceedings of the fourth Berkeley symposium on mathematical statistics and probability 4, 321-333. 
[46] Van der Leeuw, J., 1994, The covariance matrix of ARMA errors in closed form, Journal of econometrics $63,397-405$.

[47] Zellner, A., 1971, An introduction to Bayesian inference in econometrics, Wiley, New York. 


\section{Q Queen Mary \\ University of London}

This working paper has been produced by the Department of Economics at Queen Mary, University of London

Copyright $\odot 2000$ Hugo Kruiniger All rights reserved.

\section{Department of Economics}

Queen Mary, University of London

Mile End Road

London E1 4NS

Tel: +44 (0)20 78825096 or Fax: +44 (0)20 89833580

Email: j.conner@qmw.ac.uk

Website: www.econ.qmw.ac.uk/papers/wp.htm 